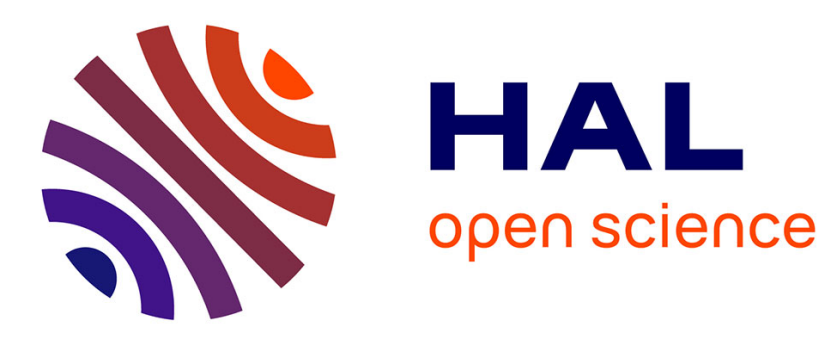

\title{
The Effective Vlasov-Poisson System for the Finite Larmor Radius Regime
}

\author{
Mihai Bostan, Aurélie Finot
}

\section{To cite this version:}

Mihai Bostan, Aurélie Finot. The Effective Vlasov-Poisson System for the Finite Larmor Radius Regime. Multiscale Modeling and Simulation: A SIAM Interdisciplinary Journal, 2016, 14 (4), pp.1238

- 1275. 10.1137/16M1060479. hal-01475672

\section{HAL Id: hal-01475672 \\ https://hal.science/hal-01475672}

Submitted on 24 Feb 2017

HAL is a multi-disciplinary open access archive for the deposit and dissemination of scientific research documents, whether they are published or not. The documents may come from teaching and research institutions in France or abroad, or from public or private research centers.
L'archive ouverte pluridisciplinaire HAL, est destinée au dépôt et à la diffusion de documents scientifiques de niveau recherche, publiés ou non, émanant des établissements d'enseignement et de recherche français ou étrangers, des laboratoires publics ou privés. 


\title{
The effective Vlasov-Poisson system for the finite Larmor radius regime
}

\author{
Mihaï BOSTAN *, Aurélie FINOT ${ }^{\dagger}$
}

(May 25, 2016)

\begin{abstract}
The subject matter of this paper concerns the finite Larmor radius regime of the Vlasov-Poisson system for strongly magnetized plasmas. We appeal to gyro-average methods, and determine the explicit expressions for the velocity and acceleration fields in the limit Vlasov equation. We investigate the Hamiltonian structure of the limit model (trajectories), analyse its properties (conservations of the mass, kinetic energy, electric energy) and justify rigorously the asymptotic behavior, following the formal arguments developed in ${ }^{1}$.
\end{abstract}

Keywords: Vlasov-Poisson system, Averaging, Finite Larmor radius approximation.

AMS classification: 35Q75, 78A35, 82D10.

\section{Introduction}

One of the main application of the tokamak plasmas relies on the energy production through the magnetic confinement. We study the dynamics of a population of charged particles under the action of a strong magnetic field, whose role is to ensure the confinement around the magnetic lines. An interesting model is the so called finite Larmor radius regime, that is, we assume that the particle distribution fluctuates at the Larmor circle length scale $l=2 \pi \rho_{L}$

\footnotetext{
*Institut de Mathématiques de Marseille, Centre de Mathématiques et Informatique, UMR 7373, 39 rue Frédéric Joliot Curie, 13453 Marseille Cedex 13 France. E-mail : mihai.bostan@univ-amu.fr

${ }^{\dagger}$ Institut de Mathématiques de Marseille, Centre de Mathématiques et Informatique, UMR 7373, 39 rue Frédéric Joliot Curie, 13453 Marseille Cedex 13 France. E-mail : aurelie.finot@univ-amu.fr.

${ }^{1}$ M. Bostan, A. Finot, M. Hauray, The effective Vlasov-Poisson system for strongly magnetized plasmas, C. R. Acad. Sci. Paris, Ser. I(2016), http://dx.doi.org/10.1016/j.crma.2016.04.014
} 
along the orthogonal directions, but at a much larger scale in the parallel direction with respect to the magnetic field $[10,11,13]$. For simplicity we consider a strong uniform magnetic field $\mathbf{B}^{\varepsilon}=\left(0,0, B^{\varepsilon}\right)$, perpendicular to $x_{1} O x_{2}$. We use the notations

$$
\bar{x}=\left(x_{1}, x_{2}\right), \quad \bar{v}=\left(v_{1}, v_{2}\right),{ }^{\perp} \bar{v}=\left(v_{2},-v_{1}\right), \quad\left(x_{1}, x_{2}\right),\left(v_{1}, v_{2}\right) \in \mathbb{R}^{2} .
$$

The assumptions of our regime are

1. The reference time $T$ is much larger than the cyclotronic period (strong magnetic field) i.e.,

$$
T \frac{\left|q B^{\varepsilon}\right|}{2 \pi m}=\frac{1}{\varepsilon}, \text { with } 0<\varepsilon<<1 .
$$

Notice that the above hypothesis writes also $\frac{T V}{2 \pi \rho_{L}}=\frac{1}{\varepsilon}$, where $V$ is the reference velocity, and $\rho_{L}$ is the typical Larmor radius.

2. The kinetic energy is much larger than the potential energy

$$
\frac{m V^{2}}{q \phi}=\frac{1}{\varepsilon}
$$

where $m$ is the particle mass, $q$ is the particle charge, and $\phi$ is the reference electric potential.

3. The typical length of the electric phenomena coincides with the Larmor circle length i.e.,

$$
\frac{\varepsilon_{0} \phi}{n q}=l^{2}
$$

Here $\varepsilon_{0}$ is the electric permittivity of the vacuum and $n$ is the average charge concentration.

Notice that the scaling in (1) is not so relevant for tokamak plasmas. Indeed, a much interesting assumption would be to consider that the Debye length is much smaller with respect to the Larmor radius, leading to quasi-neutral regimes. We expect that the method employed here will apply to other (more complex) regimes, including quasi-neutrality. This will be the topic of future works.

The presence density $f^{\varepsilon}=f^{\varepsilon}(t, x, v)$ and the electric potential $\phi^{\varepsilon}$ satisfy the following Vlasov-Poisson system, up to a multiplicative constant $\omega_{c}$, of order one

$$
\begin{gathered}
\partial_{t} f^{\varepsilon}+\frac{1}{\varepsilon}\left(\bar{v} \cdot \nabla_{\bar{x}} f^{\varepsilon}+\omega_{c}{ }^{\perp} \bar{v} \cdot \nabla_{\bar{v}} f^{\varepsilon}\right)+v_{3} \partial_{x_{3}} f^{\varepsilon}-\nabla_{\bar{x}} \phi^{\varepsilon} \cdot \nabla_{\bar{v}} f^{\varepsilon}-\varepsilon \partial_{x_{3}} \phi^{\varepsilon} \partial_{v_{3}} f^{\varepsilon}=0,(t, x, v) \in \mathbb{R}_{+} \times \mathbb{R}^{3} \times \mathbb{R}^{3} \\
-\Delta_{\bar{x}} \phi^{\varepsilon}-\varepsilon^{2} \partial_{x_{3}}^{2} \phi^{\varepsilon}=\rho^{\varepsilon}:=\int_{\mathbb{R}^{3}} f^{\varepsilon}(t, x, v) \mathrm{d} v, \quad(t, x) \in \mathbb{R}_{+} \times \mathbb{R}^{3}
\end{gathered}
$$




$$
f^{\varepsilon}(0, x, v)=f^{\text {in }}(x, v), \quad(x, v) \in \mathbb{R}^{3} \times \mathbb{R}^{3} .
$$

We introduce the notations $T_{c}=2 \pi / \omega_{c}, \omega_{c}^{\varepsilon}=\omega_{c} / \varepsilon, T_{c}^{\varepsilon}=\frac{2 \pi}{\omega_{c}^{\varepsilon}}=\varepsilon \frac{2 \pi}{\omega_{c}}=\varepsilon T_{c}$.

We investigate the asymptotic behavior of $\left(f^{\varepsilon}, \phi^{\varepsilon}\right)_{\varepsilon>0}$ when $\varepsilon$ becomes small. Clearly we are faced to a two time scale problem : some quantities change every cyclotronic period, some other are left invariant during a cyclotronic period. For example, the Larmor center $\bar{x}+\frac{\perp_{\bar{v}}}{\omega_{c}}$ is an invariant of the fast cyclotronic motion. Indeed, the characteristic system of the Vlasov equation (2) writes

$$
\begin{gathered}
\frac{\mathrm{d} \bar{X}^{\varepsilon}}{\mathrm{d} t}=\frac{\bar{V}^{\varepsilon}(t)}{\varepsilon}, \quad \frac{\mathrm{d} \bar{V}^{\varepsilon}}{\mathrm{d} t}=\omega_{c} \frac{{ }^{\perp} \bar{V}^{\varepsilon}(t)}{\varepsilon}-\nabla_{\bar{x}} \phi^{\varepsilon}\left(t, X^{\varepsilon}(t)\right) \\
\frac{\mathrm{d} X_{3}^{\varepsilon}}{\mathrm{d} t}=V_{3}^{\varepsilon}(t), \quad \frac{\mathrm{d} V_{3}^{\varepsilon}}{\mathrm{d} t}=-\varepsilon \partial_{x_{3}} \phi^{\varepsilon}\left(t, X^{\varepsilon}(t)\right) \\
X^{\varepsilon}(0 ; x, v)=x, \quad V^{\varepsilon}(0 ; x, v)=v
\end{gathered}
$$

and therefore, the time derivative of the Larmor center is given by

$$
\frac{\mathrm{d}}{\mathrm{d} t}\left(\bar{X}^{\varepsilon}+\frac{\perp \bar{V}^{\varepsilon}}{\omega_{c}}\right)=\frac{\bar{V}^{\varepsilon}}{\varepsilon}+\frac{1}{\omega_{c}} \perp\left(\frac{\omega_{c}}{\varepsilon} \perp \bar{V}^{\varepsilon}(t)-\nabla_{\bar{x}} \phi^{\varepsilon}\left(t, X^{\varepsilon}(t)\right)\right)=-\frac{{ }^{\perp} \nabla_{\bar{x}} \phi^{\varepsilon}\left(t, X^{\varepsilon}(t)\right)}{\omega_{c}} .
$$

The variation of the Larmor center over one cyclotronic period $T_{c}^{\varepsilon}=\varepsilon T_{c}$ is of order $\varepsilon$, and thus negligible. The Larmor center is left invariant with respect to the fast dynamics. Similarly we obtain

$$
\begin{aligned}
\frac{\mathrm{d}}{\mathrm{d} t}\left\{\mathcal{R}\left(\omega_{c} t / \varepsilon\right) \bar{V}^{\varepsilon}(t)\right\} & =-\frac{\omega_{c}}{\varepsilon} \mathcal{R}\left(\omega_{c} t / \varepsilon\right){ }^{\perp} \bar{V}^{\varepsilon}(t)+\mathcal{R}\left(\omega_{c} t / \varepsilon\right)\left[\frac{\omega_{c}}{\varepsilon} \perp \bar{V}^{\varepsilon}(t)-\nabla_{\bar{x}} \phi^{\varepsilon}\left(t, X^{\varepsilon}(t)\right)\right] \\
& =-\mathcal{R}\left(\omega_{c} t / \varepsilon\right) \nabla_{\bar{x}} \phi^{\varepsilon}\left(t, X^{\varepsilon}(t)\right)
\end{aligned}
$$

saying that $\mathcal{R}\left(\omega_{c} t / \varepsilon\right) \bar{V}^{\varepsilon}(t)$ remains almost unchanged over a cyclotronic period, together with $X_{3}^{\varepsilon}(t)$ and $V_{3}^{\varepsilon}(t)$. Here $\mathcal{R}$ stands for the rotation of angle $\theta \in \mathbb{R}$. Motivated by the previous computations, at any time $t \in \mathbb{R}_{+}$, we introduce the change of coordinates $(x, v) \rightarrow(\widetilde{x}, \widetilde{v})$ given by

$$
\overline{\widetilde{x}}=\bar{x}+\frac{{ }^{\perp} \bar{v}}{\omega_{c}}, \quad \widetilde{x}_{3}=x_{3}, \quad \overline{\widetilde{v}}=\mathcal{R}\left(\omega_{c} t / \varepsilon\right) \bar{v}, \quad \widetilde{v}_{3}=v_{3} .
$$

Notice that the Jacobian determinant of this transformation equals 1, and thus the Lebesgue measure is preserved $\mathrm{d} \widetilde{v} \mathrm{~d} \widetilde{x}=\mathrm{d} v \mathrm{~d} x$. The idea is to get stability by filtering out the fast oscillations with respect to the cyclotronic motion $[5,6,7]$. Let us denote by $\tilde{f}^{\varepsilon}(t, \cdot, \cdot)$ the presence density in the coordinates $(\widetilde{x}, \widetilde{v})$, that is

$$
\tilde{f}^{\varepsilon}(t, \widetilde{x}, \widetilde{v})=f^{\varepsilon}(t, x, v), \quad \bar{x}=\overline{\widetilde{x}}-\frac{\mathcal{R}\left(-\omega_{c} t / \varepsilon\right)}{\omega_{c}} \perp \overline{\widetilde{v}}, \quad x_{3}=\widetilde{x}_{3}, \quad \bar{v}=\mathcal{R}\left(-\omega_{c} t / \varepsilon\right) \overline{\widetilde{v}}, \quad v_{3}=\widetilde{v}_{3} .
$$


Performing the above change of coordinates we deduce that the density $\tilde{f}^{\varepsilon}$ satisfies the Vlasov equation

$$
\partial_{t} \tilde{f}^{\varepsilon}-\frac{{ }^{\perp} \nabla_{\bar{x}} \phi^{\varepsilon}}{\omega_{c}} \cdot \nabla_{\overline{\widetilde{x}}} \tilde{f}^{\varepsilon}+\widetilde{v}_{3} \partial_{\widetilde{x}_{3}} \tilde{f}^{\varepsilon}-\mathcal{R}\left(\omega_{c} t / \varepsilon\right) \nabla_{\bar{x}} \phi^{\varepsilon} \cdot \nabla_{\overline{\widetilde{v}}} \tilde{f}^{\varepsilon}-\varepsilon \partial_{x_{3}} \phi^{\varepsilon} \partial_{\widetilde{v}_{3}} \tilde{f}^{\varepsilon}=0
$$

and the initial condition

$$
\tilde{f}^{\varepsilon}(0, \widetilde{x}, \widetilde{v})=f^{\text {in }}\left(\overline{\widetilde{x}}-\frac{\perp \overline{\widetilde{v}}}{\omega_{c}}, \widetilde{x}_{3}, \widetilde{v}\right) .
$$

Notice that, in the above Vlasov equation, the electric field $\left(-\nabla_{\bar{x}} \phi^{\varepsilon},-\varepsilon \partial_{x_{3}} \phi^{\varepsilon}\right)$ is to be computed at the point $x=\left(\overline{\widetilde{x}}-\omega_{c}^{-1} \mathcal{R}\left(-\omega_{c} t / \varepsilon\right) \perp \overline{\widetilde{v}}, \widetilde{x}_{3}\right)$. Observe that in this coordinates, the Vlasov equation contains no singular advection fields (the velocity and acceleration fields of (8) are exactly the time derivatives in (5), (6) and thus of order 1 , and not of order $1 / \varepsilon$ ). Therefore we expect a stability result for the family $\left(\tilde{f}^{\varepsilon}\right)_{\varepsilon}$, that is, there is a presence density profile $\tilde{f}$ such that

$$
f^{\varepsilon}(t, x, v)-\tilde{f}\left(t, \bar{x}+\frac{\perp \bar{v}}{\omega_{c}}, x_{3}, \mathcal{R}\left(\omega_{c} t / \varepsilon\right) \bar{v}, v_{3}\right)=o(1), \quad \varepsilon \searrow 0 .
$$

The well posedness of the Vlasov-Poisson system follows by standard arguments, based on uniform mass and energy estimates, with respect to $\varepsilon>0$, see $[2,4]$. The asymptotic behavior comes by performing a two scale analysis. The key point is to pick new coordinates which are left invariant with respect to the fast dynamics. This leads naturally to average transport operators whose study was detailed in previous works $[5,6,7]$. The novelty with respect to the previous approaches $[10,11]$ consists in averaging directly the non linear equation, satisfied by the presence density $f^{\varepsilon}$, resulting when replacing the self-consistent electric potential through the Poisson equation. In other words, instead of coupling two averaged equations (Vlasov and Poisson), we average the fully non linear coupling between the Vlasov and Poisson equations. This allows us to emphasize the Hamiltonian structure of the effective Vlasov-Poisson model, and new conservations which characterize the finite Larmor radius regime. A very intuitive analysis is presented in Section 2, where the effective trajectories are computed by averaging formally with respect to the fast cyclotronic motion, leading to gyro-average effects. The point is that the electric field is treated as self-consistent, and thus we also need to average the fundamental solution of the Laplace operator. As the cyclotronic trajectories are circles, for averaging the fundamental solution of the Laplace operator we can appeal to the mean property of the harmonic functions.

We mention that the asymptotic regime considered in $(2),(3),(4)$ is the same as that in [13]. Nevertheless, the coordinate changes, leading to the new presence densities are different: 
in [13] a non linear change of frame is used, depending on the self-consistent electric field, which is very different with respect to the linear change (7). Accordingly, the limit models obtained in the new coordinates are different. More exactly, in the two dimensional setting i.e., $x=\left(x_{1}, x_{2}\right), v=\left(v_{1}, v_{2}\right),{ }^{\perp} v=\left(v_{2},-v_{1}\right), \widetilde{x}=x+\frac{\perp_{v}}{\omega_{c}}, \widetilde{v}=\mathcal{R}\left(\omega_{c} t / \varepsilon\right) v$, we prove cf. [8]

Theorem 1.1 Let $f^{\text {in }}=f^{\text {in }}(x, v)$ be a non negative presence density satisfying

$H 1 \quad \int_{\mathbb{R}^{2}} \int_{\mathbb{R}^{2}} f^{\text {in }}(x, v) \mathrm{d} v \mathrm{~d} x<+\infty$

H2 $\quad \int_{\mathbb{R}^{2}} \int_{\mathbb{R}^{2}} \frac{|v|^{2}}{2} f^{\text {in }}(x, v) \mathrm{d} v \mathrm{~d} x<+\infty$

H3 there is a bounded, non increasing function $F^{\mathrm{in}}=F^{\mathrm{in}}(r) \in L^{\infty} \cap L^{1}\left(\mathbb{R}_{+} ; r \mathrm{~d} r\right)$, such that $f^{\text {in }}(x, v) \leq F^{\text {in }}(|v|), \quad(x, v) \in \mathbb{R}^{2} \times \mathbb{R}^{2}$.

We consider the family $\left(f^{\varepsilon}, \phi^{\varepsilon}\right)_{\varepsilon>0}$ of weak solutions for the Vlasov-Poisson system

$$
\begin{gathered}
\partial_{t} f^{\varepsilon}+\frac{1}{\varepsilon}\left(v \cdot \nabla_{x} f^{\varepsilon}+\omega_{c}{ }^{\perp} v \cdot \nabla_{v} f^{\varepsilon}\right)-\nabla_{x} \phi^{\varepsilon} \cdot \nabla_{v} f^{\varepsilon}=0, \quad(t, x, v) \in \mathbb{R}_{+} \times \mathbb{R}^{2} \times \mathbb{R}^{2} \\
-\Delta_{x} \phi^{\varepsilon}=\rho^{\varepsilon}(t, x):=\int_{\mathbb{R}^{2}} f^{\varepsilon}(t, x, v) \mathrm{d} v, \quad(t, x) \in \mathbb{R}_{+} \times \mathbb{R}^{2} \\
f^{\varepsilon}(0, x, v)=f^{\text {in }}(x, v), \quad(x, v) \in \mathbb{R}^{2} \times \mathbb{R}^{2}
\end{gathered}
$$

and we denote by $\left(\tilde{f}^{\varepsilon}\right)_{\varepsilon>0}$ the densities

$$
\tilde{f}^{\varepsilon}(t, \widetilde{x}, \widetilde{v})=f^{\varepsilon}\left(t, \widetilde{x}-\frac{\mathcal{R}\left(-\omega_{c} t / \varepsilon\right)}{\omega_{c}} \perp \widetilde{v}, \mathcal{R}\left(-\omega_{c} t / \varepsilon\right) \widetilde{v}\right), \quad(t, \widetilde{x}, \widetilde{v}) \in \mathbb{R}_{+} \times \mathbb{R}^{2} \times \mathbb{R}^{2}, \quad \varepsilon>0 .
$$

Therefore there is a sequence $\left(\varepsilon_{k}\right)_{k}$ converging to 0 such that $\left(\tilde{f}^{\varepsilon_{k}}\right)_{k}$ converges strongly in $L^{2}\left([0, T] ; L^{2}\left(\mathbb{R}^{2} \times \mathbb{R}^{2}\right)\right)$, for any $T \in \mathbb{R}_{+}$, toward a solution $\tilde{f}$ of the problem

$$
\partial_{t} \tilde{f}+\mathcal{V}[\tilde{f}(t)](\widetilde{x}, \widetilde{v}) \cdot \nabla_{\widetilde{x}} \tilde{f}+\mathcal{A}[\tilde{f}(t)](\widetilde{x}, \widetilde{v}) \cdot \nabla_{\widetilde{v}} \tilde{f}=0, \quad(t, \widetilde{x}, \widetilde{v}) \in \mathbb{R}_{+} \times \mathbb{R}^{2} \times \mathbb{R}^{2}
$$

with the initial condition

$$
\tilde{f}(0, \widetilde{x}, \widetilde{v})=f^{\text {in }}\left(\widetilde{x}-\frac{\perp \widetilde{v}}{\omega_{c}}, \widetilde{v}\right),(\widetilde{x}, \widetilde{v}) \in \mathbb{R}^{2} \times \mathbb{R}^{2}
$$

where the velocity and acceleration vector fields $\mathcal{V}, \mathcal{A}$ are given by

$$
\begin{gathered}
\mathcal{V}[\tilde{f}(t)](\widetilde{x}, \widetilde{v})=-\omega_{c}^{-1}{ }^{\perp} \nabla_{\widetilde{x}} \tilde{\phi}[\tilde{f}(t)], \quad \mathcal{A}[\tilde{f}(t)](\widetilde{x}, \widetilde{v})=\omega_{c}{ }^{\perp} \nabla_{\widetilde{v}} \tilde{\phi}[\tilde{f}(t)] \\
\tilde{\phi}[\tilde{f}(t)]=-\frac{1}{2 \pi} \int_{\mathbb{R}^{2}} \int_{\mathbb{R}^{2}}\left\{\ln \frac{|\widetilde{v}-\widetilde{w}|}{\left|\omega_{c}\right|} \mathbf{1}_{\left\{|\widetilde{x}-\widetilde{y}| \leq \frac{|\widetilde{v}-\widetilde{w}|}{\left|\omega_{c}\right|}\right\}}+\ln |\widetilde{x}-\widetilde{y}| \mathbf{1}_{\left\{|\widetilde{x}-\widetilde{y}|>\frac{|\widetilde{v}-\widetilde{w}|}{\left|\omega_{c}\right|}\right\}}\right\} \tilde{f}(t, \widetilde{y}, \widetilde{w}) \mathrm{d} \tilde{w} \mathrm{~d} \tilde{y}
\end{gathered}
$$


The asymptotic behavior of (9), (10), (11) has already been studied before. In $[10,11,13]$ the authors appeal to the two scale convergence method. Nevertheless, the fast time variable persists in the limit model, and the computation of the velocity and acceleration vector fields of the limit Vlasov equation requires the resolution of a Poisson equation for every couple of slow/fast time variables, and some averaging procedure. In [5] the author obtained a convergence result towards a simpler model, which is valid only for well-prepared initial data. Our result applies to general initial data, and the limit model is a rather simple equation. It is a fully explicit non linear transport equation, whose characteristic system is Hamiltonian (with respect to the appropriate variables cf. Proposition 2.1) and which can be studied in a much simpler way. Roughly speaking, the fast time variable appearing in the previous works is averaged in a fully explicit way. Notice that the velocity and acceleration fields $\mathcal{V}, \mathcal{A}$ are divergence free. Therefore (12) writes into conservative form, which guarantees the mass conservation. More generally we prove.

\section{Proposition 1.1}

1. Let $\tilde{f}=\tilde{f}(t, \widetilde{x}, \widetilde{v})$ be a solution of the problem (12), (14), (15) such that $1, \widetilde{x}, \widetilde{v},|\widetilde{x}|^{2},|\widetilde{v}|^{2}$ are integrable functions with respect to $\tilde{f}(0, \widetilde{x}, \widetilde{v}) \mathrm{d} \widetilde{v} \mathrm{~d} \widetilde{x}=f^{\text {in }}\left(\widetilde{x}-\omega_{c}^{-1 \perp} \widetilde{v}, \widetilde{v}\right) \mathrm{d} \widetilde{v} \mathrm{~d} \widetilde{x}$. For any $t \in \mathbb{R}_{+}$we have

$$
\int_{\mathbb{R}^{2}} \int_{\mathbb{R}^{2}}\left\{1, \widetilde{x}, \widetilde{v},|\widetilde{x}|^{2},|\widetilde{v}|^{2}\right\} \tilde{f}(t, \widetilde{x}, \widetilde{v}) \mathrm{d} \tilde{v} \mathrm{~d} \tilde{x}=\int_{\mathbb{R}^{2}} \int_{\mathbb{R}^{2}}\left\{1, \widetilde{x}, \widetilde{v},|\widetilde{x}|^{2},|\widetilde{v}|^{2}\right\} f^{\text {in }}\left(\widetilde{x}-\omega_{c}^{-1} \perp \widetilde{v}, \widetilde{v}\right) \mathrm{d} \tilde{v} \mathrm{~d} \tilde{x} .
$$

2. Let $\tilde{f}=\tilde{f}(t, \widetilde{x}, \widetilde{v})$ be a solution of the problem (12), (14), (15) such that

$$
\frac{1}{2} \int_{\mathbb{R}^{2}} \int_{\mathbb{R}^{2}} \tilde{\phi}[\tilde{f}(0)](\widetilde{x}, \widetilde{v}) \tilde{f}(0, \widetilde{x}, \widetilde{v}) \mathrm{d} \tilde{v} \mathrm{~d} \tilde{x}<+\infty .
$$

The electric energy is preserved in time

$$
\frac{\mathrm{d}}{\mathrm{d} t} \frac{1}{2} \int_{\mathbb{R}^{2}} \int_{\mathbb{R}^{2}} \tilde{\phi}[\tilde{f}(t)](\widetilde{x}, \widetilde{v}) \tilde{f}(t, \widetilde{x}, \widetilde{v}) \mathrm{d} \tilde{v} \mathrm{~d} \tilde{x}=0, \quad t \in \mathbb{R}_{+} .
$$

The two dimensional analysis extends easily to the three dimensional setting, at least formally. Following the same arguments one gets the result.

Theorem 1.2 Let $f^{\text {in }}=f^{\text {in }}(x, v)$ be a non negative presence density with finite mass and kinetic energy, and bounded charge density $\rho^{\mathrm{in}}(x):=\int_{\mathbb{R}^{3}} f^{\mathrm{in}}(x, v) \mathrm{d} v$. We consider the family $\left(f^{\varepsilon}, \phi^{\varepsilon}\right)_{\varepsilon}$ of weak solutions for the Vlasov-Poisson problems (2), (3), (4) and we denote by $\left(\tilde{f}^{\varepsilon}\right)_{\varepsilon>0}$ the densities

$\tilde{f}^{\varepsilon}(t, \widetilde{x}, \widetilde{v})=f^{\varepsilon}\left(t, \overline{\widetilde{x}}-\frac{\mathcal{R}\left(-\omega_{c} t / \varepsilon\right)}{\omega_{c}} \perp \overline{\widetilde{v}}, \widetilde{x}_{3}, \mathcal{R}\left(-\omega_{c} t / \varepsilon\right) \overline{\widetilde{v}}, \widetilde{v}_{3}\right), \quad(t, \widetilde{x}, \widetilde{v}) \in \mathbb{R}_{+} \times \mathbb{R}^{3} \times \mathbb{R}^{3}, \quad \varepsilon>0$. 
Therefore the limit density of the family $\left(\tilde{f}^{\varepsilon}\right)_{\varepsilon}$ solves the problem

$$
\begin{gathered}
\partial_{t} \tilde{f}+\overline{\mathcal{V}}\left[\tilde{f}\left(t, \widetilde{x}_{3}\right)\right] \cdot \nabla_{\overline{\widetilde{x}}} \tilde{f}+\widetilde{v}_{3} \partial_{\widetilde{x}_{3}} \tilde{f}+\overline{\mathcal{A}}\left[\tilde{f}\left(t, \widetilde{x}_{3}\right)\right] \cdot \nabla_{\overline{\widetilde{v}}} \tilde{f}=0, \quad(t, \widetilde{x}, \widetilde{v}) \in \mathbb{R}_{+} \times \mathbb{R}^{3} \times \mathbb{R}^{3} \\
\tilde{f}(0, \widetilde{x}, \widetilde{v})=f^{\text {in }}\left(\overline{\widetilde{x}}-\frac{\perp \overline{\widetilde{v}}}{\omega_{c}}, \widetilde{x}_{3}, \widetilde{v}\right), \quad(\widetilde{x}, \widetilde{v}) \in \mathbb{R}^{3} \times \mathbb{R}^{3}
\end{gathered}
$$

where the velocity and acceleration fields are given by

$$
\begin{aligned}
& \overline{\mathcal{V}}[\tilde{f}]=-\omega_{c}^{-1}{ }^{\perp} \nabla_{\overline{\tilde{x}}} \tilde{\phi}[\tilde{f}], \quad \overline{\mathcal{A}}[\tilde{f}]=\omega_{c}{ }^{\perp} \nabla_{\overline{\tilde{v}}} \tilde{\phi}[\tilde{f}] \\
& \tilde{\phi}\left[\tilde{f}\left(t, \widetilde{x}_{3}\right)\right](\overline{\widetilde{x}}, \overline{\widetilde{v}})=-\frac{1}{2 \pi} \int_{\mathbb{R}^{2}} \int_{\mathbb{R}^{3}}\left\{\ln \frac{|\overline{\widetilde{v}}-\overline{\widetilde{w}}|}{\left|\omega_{c}\right|} \mathbf{1}_{\left\{|\overline{\widetilde{x}}-\overline{\widetilde{y}}| \leq \frac{|\overline{\tilde{v}}-\overline{\widetilde{w}}|}{\left|\omega_{c}\right|}\right\}}+\ln |\overline{\widetilde{x}}-\overline{\widetilde{y}}| \mathbf{1}_{\left\{|\overline{\widetilde{x}}-\overline{\tilde{y}}|>\frac{|\overline{\tilde{v}}-\overline{\tilde{v}}|}{\left|\omega_{c}\right|}\right\}}\right\} \\
& \times \tilde{f}\left(t, \overline{\widetilde{y}}, \widetilde{x}_{3}, \widetilde{w}\right) \mathrm{d} \widetilde{w} \mathrm{~d} \overline{\widetilde{y}} .
\end{aligned}
$$

The above considerations extend to more general situations. Periodic spatial domains can be considered. In this case we need to average the fundamental solution of the Laplace operator with periodic boundary conditions. Another generalization concerns the magnetic field geometry. As long as the invariants of the cyclotronic motion are available (which play a crucial role when defining the new coordinates), the arguments still apply and allow us to justify the finite Larmor radius regime. It happens that for quite a large class of magnetic fields, such invarianst are well defined and thus our method adapts in this cases. A complete study of these aspects will be discussed soon.

Our paper is organized as follows. In Section 2 we investigate the two dimensional case. We compute the effective trajectories and we establish the main properties of the limit VlasovPoisson system. Section 3 is devoted to convergence results. We proceed by a two scale analysis. The three dimensional setting is discussed in Section 4. Some technical arguments are presented in Appendix A.

\section{The two dimensional setting}

In this section we focus on the bi-dimensional case. We use the notations $x=\left(x_{1}, x_{2}\right), v=$ $\left(v_{1}, v_{2}\right),{ }^{\perp} v=\left(v_{2},-v_{1}\right), \widetilde{x}=x+\frac{\perp_{v}}{\omega_{c}}, \widetilde{v}=\mathcal{R}\left(\omega_{c} t / \varepsilon\right) v$. We investigate the asymptotic behavior of the solutions $\left(f^{\varepsilon}, \phi^{\varepsilon}\right)_{\varepsilon>0}$ of the problems (9), (10), (11). We start our work by formal computations at the characteristic equation level. Later on we complete our analysis by rigorous arguments. The trajectories in the phase space $(x, v)$ oscillate at the cyclotronic frequency $\omega_{c}^{\varepsilon}=\omega_{c} / \varepsilon$

$$
\frac{\mathrm{d} X^{\varepsilon}}{\mathrm{d} t}=\frac{V^{\varepsilon}(t)}{\varepsilon}, \quad \frac{\mathrm{d} V^{\varepsilon}}{\mathrm{d} t}=\frac{\omega_{c}}{\varepsilon}{ }^{\perp} V^{\varepsilon}(t)-\nabla_{x} \phi^{\varepsilon}\left(t, X^{\varepsilon}(t)\right)
$$


but the quantities $\widetilde{X}^{\varepsilon}(t)=X^{\varepsilon}(t)+{ }^{\perp} V^{\varepsilon}(t) / \omega_{c}, \widetilde{V}^{\varepsilon}(t)=\mathcal{R}\left(\omega_{c} t / \varepsilon\right) V^{\varepsilon}(t)$ are left invariant with respect to the cyclotronic dynamics

$$
\frac{\mathrm{d} \widetilde{X}^{\varepsilon}}{\mathrm{d} t}=-\frac{{ }^{\perp} \nabla_{x} \phi^{\varepsilon}\left(t, X^{\varepsilon}(t)\right)}{\omega_{c}}, \frac{\mathrm{d} \widetilde{V}^{\varepsilon}}{\mathrm{d} t}=-\mathcal{R}\left(\omega_{c} t / \varepsilon\right) \nabla_{x} \phi^{\varepsilon}\left(t, X^{\varepsilon}(t)\right) .
$$

We expect that the family of trajectories $\left(\widetilde{X}^{\varepsilon}, \widetilde{V}^{\varepsilon}\right)_{\varepsilon>0}$ is stable as $\varepsilon$ becomes small, and we are looking for the limit trajectory $(\tilde{X}, \widetilde{V})=\lim _{\varepsilon} \searrow_{0}\left(\widetilde{X}^{\varepsilon}, \widetilde{V}^{\varepsilon}\right)$. Once we have determined the limit characteristic equations, let us say

$$
\frac{\mathrm{d} \widetilde{X}}{\mathrm{~d} t}=\mathcal{V}(t, \widetilde{X}(t), \widetilde{V}(t)), \frac{\mathrm{d} \widetilde{V}}{\mathrm{~d} t}=\mathcal{A}(t, \widetilde{X}(t), \widetilde{V}(t))
$$

for some velocity field $\mathcal{V}$ and acceleration field $\mathcal{A}$, we solve for

$$
\begin{gathered}
\partial_{t} \tilde{f}+\mathcal{V} \cdot \nabla_{\widetilde{x}} \tilde{f}+\mathcal{A} \cdot \nabla_{\widetilde{v}} \tilde{f}=0, \quad(t, \widetilde{x}, \widetilde{v}) \in \mathbb{R}_{+} \times \mathbb{R}^{2} \times \mathbb{R}^{2} \\
\tilde{f}(0, \widetilde{x}, \widetilde{v})=f^{\text {in }}\left(\widetilde{x}-\frac{{ }^{\perp} \widetilde{v}}{\omega_{c}}, \widetilde{v}\right), \quad(\widetilde{x}, \widetilde{v}) \in \mathbb{R}^{2} \times \mathbb{R}^{2} .
\end{gathered}
$$

We introduce the presence densities in the phase space $(\widetilde{x}, \widetilde{v})$ given by

$$
\tilde{f}^{\varepsilon}(t, \widetilde{x}, \widetilde{v})=f^{\varepsilon}(t, x, v), \quad \widetilde{x}=x+\frac{{ }^{\perp} v}{\omega_{c}}, \widetilde{v}=\mathcal{R}\left(\omega_{c} t / \varepsilon\right) v
$$

which satisfy the Vlasov equations see (8)

$$
\partial_{t} \tilde{f}^{\varepsilon}-\frac{{ }^{\perp} \nabla_{x} \phi^{\varepsilon}}{\omega_{c}} \cdot \nabla_{\widetilde{x}} \tilde{f}^{\varepsilon}-\mathcal{R}\left(\omega_{c} t / \varepsilon\right) \nabla_{x} \phi^{\varepsilon} \cdot \nabla_{\widetilde{v}} \tilde{f}^{\varepsilon}=0, \quad(t, \widetilde{x}, \widetilde{v}) \in \mathbb{R}_{+} \times \mathbb{R}^{2} \times \mathbb{R}^{2}
$$

and the initial condition

$$
\tilde{f}^{\varepsilon}(0, \widetilde{x}, \widetilde{v})=f^{\text {in }}\left(\widetilde{x}-\frac{\perp \widetilde{v}}{\omega_{c}}, \widetilde{v}\right),(\widetilde{x}, \widetilde{v}) \in \mathbb{R}^{2} \times \mathbb{R}^{2} .
$$

We expect that the family $\left(\tilde{f}^{\varepsilon}\right)_{\varepsilon>0}$ is stable when $\varepsilon$ becomes small and we denote by $\tilde{f}^{0}$ the expected limit density, as $\varepsilon \searrow 0$. We claim that $\tilde{f}=\tilde{f}^{0}$, which will imply that

$$
f^{\varepsilon}(t, x, v)=\tilde{f}^{\varepsilon}\left(t, x+\frac{{ }^{\perp} v}{\omega_{c}}, \mathcal{R}\left(\omega_{c} t / \varepsilon\right) v\right) \approx \tilde{f}\left(t, x+\frac{{ }^{\perp} v}{\omega_{c}}, \mathcal{R}\left(\omega_{c} t / \varepsilon\right) v\right) .
$$

Indeed, we have for any $\varepsilon>0$

$$
\tilde{f}^{\varepsilon}\left(t, \widetilde{X}^{\varepsilon}(t), \widetilde{V}^{\varepsilon}(t)\right)=f^{\varepsilon}\left(t, X^{\varepsilon}(t), V^{\varepsilon}(t)\right)=f(0, x, v)=f^{\text {in }}\left(\widetilde{x}-\frac{{ }^{\perp} \widetilde{v}}{\omega_{c}}, \widetilde{v}\right), \quad(\widetilde{x}, \widetilde{v}) \in \mathbb{R}^{2} \times \mathbb{R}^{2} .
$$

By passing to the limit when $\varepsilon \searrow 0$, we obtain

$$
\tilde{f}^{0}(t, \widetilde{X}(t), \widetilde{V}(t))=f^{\text {in }}\left(\widetilde{x}-\frac{\perp \widetilde{v}}{\omega_{c}}, \widetilde{v}\right)=\tilde{f}(0, \widetilde{x}, \widetilde{v})=\tilde{f}(t, \widetilde{X}(t), \widetilde{V}(t)), \quad(t, \widetilde{x}, \widetilde{v}) \in \mathbb{R}_{+} \times \mathbb{R}^{2} \times \mathbb{R}^{2}
$$

saying that $\lim _{\varepsilon \searrow 0} \tilde{f}^{\varepsilon}=\tilde{f}^{0}=\tilde{f}$. By the previous considerations, in order to determine the asymptotic behavior of the Vlasov-Poisson problem (9), (10), (11), we need to analyze the stability of the trajectories $\left(\widetilde{X}^{\varepsilon}, \widetilde{V}^{\varepsilon}\right)_{\varepsilon>0}$, when $\varepsilon \searrow 0$. 


\subsection{The effective trajectories}

We compute the velocity and acceleration fields $\mathcal{V}, \mathcal{A}$ corresponding to the limit trajectories $(\widetilde{X}, \widetilde{V})$. Part of the arguments developed in this section are formal. Nevertheless, a rigorous proof is presented in Section 3. Let us introduce the fundamental solution of the Laplace operator in $\mathbb{R}^{2}$

$$
e(z)=-\frac{1}{2 \pi} \ln |z|, \quad z \in \mathbb{R}^{2} \backslash\{0\}
$$

that is $-\Delta e=\delta_{0}$ in $\mathcal{D}^{\prime}\left(\mathbb{R}^{2}\right)$. The solution of the Poisson equation (10) writes

$$
\phi^{\varepsilon}(t, x)=\int_{\mathbb{R}^{2}} e(x-y) \rho^{\varepsilon}(t, y) \mathrm{d} y=\int_{\mathbb{R}^{2}} \int_{\mathbb{R}^{2}} e(x-y) f^{\varepsilon}(t, y, w) \mathrm{d} w \mathrm{~d} y, \quad(t, x) \in \mathbb{R}_{+} \times \mathbb{R}^{2} .
$$

Replacing the expression of the electric potential in (16) leads to

$$
\begin{aligned}
\frac{\mathrm{d} \widetilde{X}^{\varepsilon}}{\mathrm{d} t} & =-\frac{1}{\omega_{c}} \int_{\mathbb{R}^{2}} \int_{\mathbb{R}^{2}}{ }^{\perp} \nabla e\left(X^{\varepsilon}(t)-y\right) f^{\varepsilon}(t, y, w) \mathrm{d} w \mathrm{~d} y \\
& =-\frac{1}{\omega_{c}} \int_{\mathbb{R}^{2}} \int_{\mathbb{R}^{2}}{ }^{\perp} \nabla e\left(\widetilde{X}^{\varepsilon}(t)-\widetilde{y}-\frac{1}{\omega_{c}} \mathcal{R}\left(-\omega_{c} t / \varepsilon\right)^{\perp}\left(\widetilde{V}^{\varepsilon}(t)-\widetilde{w}\right)\right) \tilde{f}^{\varepsilon}(t, \widetilde{y}, \widetilde{w}) \mathrm{d} \tilde{w} \mathrm{~d} \tilde{y}
\end{aligned}
$$

and

$$
\begin{aligned}
\frac{\mathrm{d} \widetilde{V}^{\varepsilon}}{\mathrm{d} t} & =-\mathcal{R}\left(\omega_{c} t / \varepsilon\right) \int_{\mathbb{R}^{2}} \int_{\mathbb{R}^{2}} \nabla e\left(X^{\varepsilon}(t)-y\right) f^{\varepsilon}(t, y, w) \mathrm{d} w \mathrm{~d} y \\
& =-\mathcal{R}\left(\omega_{c} t / \varepsilon\right) \int_{\mathbb{R}^{2}} \int_{\mathbb{R}^{2}} \nabla e\left(\widetilde{X}^{\varepsilon}(t)-\widetilde{y}-\frac{1}{\omega_{c}} \mathcal{R}\left(-\omega_{c} t / \varepsilon\right)^{\perp}\left(\widetilde{V}^{\varepsilon}(t)-\widetilde{w}\right)\right) \tilde{f}^{\varepsilon}(t, \widetilde{y}, \widetilde{w}) \mathrm{d} \tilde{w} \mathrm{~d} \tilde{y} .
\end{aligned}
$$

We intend to average (18), (19) over one cyclotronic period $\left[t, t+T_{c}^{\varepsilon}\right]$, with $T_{c}^{\varepsilon}=\varepsilon \frac{2 \pi}{\omega_{c}}$. Integrating for $\tau \in\left[t, t+T_{c}^{\varepsilon}\right]$, and introducing the fast variable $s=(\tau-t) / \varepsilon \in\left[0, T_{c}\right]$, yield

$$
\frac{\widetilde{X}^{\varepsilon}\left(t+T_{c}^{\varepsilon}\right)-\widetilde{X}^{\varepsilon}(t)}{T_{c}^{\varepsilon}}=-\frac{1}{\omega_{c} T_{c}^{\varepsilon}} \int_{t}^{t+T_{c}^{\varepsilon}} \int_{\mathbb{R}^{2}} \int_{\mathbb{R}^{2}}{ }^{\perp} \nabla e\left(\widetilde{X}^{\varepsilon}(\tau)-\widetilde{y}-\mathcal{R}\left(-\frac{\omega_{c} \tau}{\varepsilon}\right) \frac{{ }^{\perp}\left(\widetilde{V}^{\varepsilon}(\tau)-\widetilde{w}\right)}{\omega_{c}}\right)
$$

$\times \tilde{f}^{\varepsilon}(\tau, \widetilde{y}, \widetilde{w}) \mathrm{d} \tilde{w} \mathrm{~d} \tilde{y} \mathrm{~d} \tau$

$$
=-\frac{1}{\omega_{c} T_{c}} \int_{0}^{T_{c}} \int_{\mathbb{R}^{2}} \int_{\mathbb{R}^{2}}{ }^{\perp} \nabla e\left(\widetilde{X}^{\varepsilon}(t+\varepsilon s)-\widetilde{y}-\mathcal{R}\left(-\frac{\omega_{c} t}{\varepsilon}-\omega_{c} s\right) \frac{\perp\left(\widetilde{V}^{\varepsilon}(t+\varepsilon s)-\widetilde{w}\right)}{\omega_{c}}\right)
$$

$\times \tilde{f}^{\varepsilon}(t+\varepsilon s, \widetilde{y}, \widetilde{w}) \mathrm{d} \tilde{w} \mathrm{~d} \tilde{y} \mathrm{~d} s$

$=-\frac{1}{\omega_{c} T_{c}} \int_{0}^{T_{c}} \int_{\mathbb{R}^{2}} \int_{\mathbb{R}^{2}}{ }^{\perp} \nabla e\left(\tilde{X}(t)-\widetilde{y}-\mathcal{R}\left(-\frac{\omega_{c} t}{\varepsilon}-\omega_{c} s\right) \frac{\perp(\widetilde{V}(t)-\widetilde{w})}{\omega_{c}}\right)$

$\times \tilde{f}(t, \widetilde{y}, \widetilde{w}) \mathrm{d} \tilde{w} \mathrm{~d} \tilde{y} \mathrm{~d} s+o(1)$

$$
\begin{aligned}
& =-\frac{1}{\omega_{c}} \int_{\mathbb{R}^{2}} \int_{\mathbb{R}^{2}} \frac{1}{2 \pi} \int_{0}^{2 \pi}{ }^{\perp} \nabla e\left(\tilde{X}(t)-\widetilde{y}-\mathcal{R}(\theta) \frac{\perp(\widetilde{V}(t)-\widetilde{w})}{\omega_{c}}\right) \mathrm{d} \theta \tilde{f}(t, \widetilde{y}, \widetilde{w}) \mathrm{d} \tilde{w} \mathrm{~d} \tilde{y}+o(1) \\
& =-\frac{{ }^{\perp} \nabla_{\xi}}{\omega_{c}} \int_{\mathbb{R}^{2}} \int_{\mathbb{R}^{2}} \mathcal{E}(\widetilde{X}(t)-\widetilde{y}, \widetilde{V}(t)-\widetilde{w}) \tilde{f}(t, \widetilde{y}, \widetilde{w}) \mathrm{d} \tilde{w} \mathrm{~d} \tilde{y}+o(1), \text { when } \varepsilon \searrow 0
\end{aligned}
$$


with

$$
\mathcal{E}(\xi, \eta)=\frac{1}{2 \pi} \int_{0}^{2 \pi} e\left(\xi-\omega_{c}^{-1} \mathcal{R}(\theta)^{\perp} \eta\right) \mathrm{d} \theta, \quad(\xi, \eta) \in\left(\mathbb{R}^{2} \times \mathbb{R}^{2}\right) \backslash\{(0,0)\}
$$

Passing to the limit, when $\varepsilon$ goes to 0 , and assuming that $\frac{\widetilde{X}^{\varepsilon}\left(t+T_{c}^{\varepsilon}\right)-\widetilde{X}^{\varepsilon}(t)}{T_{c}^{\varepsilon}}=\frac{\widetilde{X}\left(t+T_{c}^{\varepsilon}\right)-\widetilde{X}(t)}{T_{c}^{\varepsilon}}+o(1)$ as $\varepsilon \searrow 0$, lead to

$$
\frac{\mathrm{d} \widetilde{X}}{\mathrm{~d} t}=\mathcal{V}[\tilde{f}(t)](\tilde{X}(t), \widetilde{V}(t))
$$

where the velocity field $\mathcal{V}[\tilde{f}(t)]$ is given by

$$
\mathcal{V}[\tilde{f}(t)](\widetilde{x}, \widetilde{v})=-\frac{{ }^{\perp} \nabla_{\xi}}{\omega_{c}} \int_{\mathbb{R}^{2}} \int_{\mathbb{R}^{2}} \mathcal{E}(\widetilde{x}-\widetilde{y}, \widetilde{v}-\widetilde{w}) \tilde{f}(t, \widetilde{y}, \widetilde{w}) \mathrm{d} \tilde{w} \mathrm{~d} \tilde{y}, \quad(\widetilde{x}, \widetilde{v}) \in \mathbb{R}^{2} \times \mathbb{R}^{2} .
$$

A similar computation allows us to determine the acceleration field.

$\frac{\widetilde{V}^{\varepsilon}\left(t+T_{c}^{\varepsilon}\right)-\widetilde{V}^{\varepsilon}(t)}{T_{c}^{\varepsilon}}=-\frac{1}{T_{c}^{\varepsilon}} \int_{t}^{t+T_{c}^{\varepsilon}} \int_{\mathbb{R}^{2}} \int_{\mathbb{R}^{2}} \mathcal{R}\left(\frac{\omega_{c} \tau}{\varepsilon}\right) \nabla e\left(\tilde{X}^{\varepsilon}(\tau)-\widetilde{y}-\mathcal{R}\left(-\frac{\omega_{c} \tau}{\varepsilon}\right) \frac{\perp\left(\widetilde{V}^{\varepsilon}(\tau)-\widetilde{w}\right)}{\omega_{c}}\right)$

$\times \tilde{f}^{\varepsilon}(\tau, \widetilde{y}, \widetilde{w}) \mathrm{d} \tilde{w} \mathrm{~d} \tilde{y} \mathrm{~d} \tau$

$=-\frac{1}{T_{c}} \int_{0}^{T_{c}} \int_{\mathbb{R}^{2}} \int_{\mathbb{R}^{2}} \mathcal{R}\left(\frac{\omega_{c} t}{\varepsilon}+\omega_{c} s\right) \nabla e\left(\widetilde{X}^{\varepsilon}(t+\varepsilon s)-\widetilde{y}-\mathcal{R}\left(-\frac{\omega_{c} t}{\varepsilon}-\omega_{c} s\right) \frac{\perp\left(\widetilde{V}^{\varepsilon}(t+\varepsilon s)-\widetilde{w}\right)}{\omega_{c}}\right)$

$\times \tilde{f}^{\varepsilon}(t+\varepsilon s, \widetilde{y}, \widetilde{w}) \mathrm{d} \tilde{w} \mathrm{~d} \tilde{y} \mathrm{~d} s$

$=-\frac{1}{T_{c}} \int_{0}^{T_{c}} \int_{\mathbb{R}^{2}} \int_{\mathbb{R}^{2}} \mathcal{R}\left(\frac{\omega_{c} t}{\varepsilon}+\omega_{c} s\right) \nabla e\left(\tilde{X}(t)-\widetilde{y}-\mathcal{R}\left(-\frac{\omega_{c} t}{\varepsilon}-\omega_{c} s\right) \frac{\perp(\widetilde{V}(t)-\widetilde{w})}{\omega_{c}}\right)$

$\times \tilde{f}(t, \widetilde{y}, \widetilde{w}) \mathrm{d} \tilde{w} \mathrm{~d} \tilde{y} \mathrm{~d} s+o(1)$

$=-\int_{\mathbb{R}^{2}} \int_{\mathbb{R}^{2}} \frac{1}{2 \pi} \int_{0}^{2 \pi} \mathcal{R}(-\theta) \nabla e\left(\tilde{X}(t)-\widetilde{y}-\mathcal{R}(\theta) \frac{\perp(\widetilde{V}(t)-\widetilde{w})}{\omega_{c}}\right) \mathrm{d} \theta \tilde{f}(t, \widetilde{y}, \widetilde{w}) \mathrm{d} \tilde{w} \mathrm{~d} \tilde{y}+o(1)$

$=\omega_{c}{ }^{\perp} \nabla_{\eta} \int_{\mathbb{R}^{2}} \int_{\mathbb{R}^{2}} \mathcal{E}(\widetilde{X}(t)-\widetilde{y}, \widetilde{V}(t)-\widetilde{w}) \tilde{f}(t, \widetilde{y}, \widetilde{w}) \mathrm{d} \tilde{w} \mathrm{~d} \tilde{y}+o(1)$, when $\varepsilon \searrow 0$.

As before, the assumption $\frac{\widetilde{V}^{\varepsilon}\left(t+T_{c}^{\varepsilon}\right)-\widetilde{V}^{\varepsilon}(t)}{T_{c}^{\varepsilon}}=\frac{\widetilde{V}\left(t+T_{c}^{\varepsilon}\right)-\widetilde{V}(t)}{T_{c}^{\varepsilon}}+o(1)$ as $\varepsilon \searrow 0$, implies

$$
\frac{\mathrm{d} \widetilde{V}}{\mathrm{~d} t}=\mathcal{A}[\tilde{f}(t)](\widetilde{X}(t), \widetilde{V}(t))
$$

where the acceleration field $\mathcal{A}[\tilde{f}(t)]$ is given by

$$
\mathcal{A}[\tilde{f}(t)](\widetilde{x}, \widetilde{v})=\omega_{c}{ }^{\perp} \nabla_{\eta} \int_{\mathbb{R}^{2}} \int_{\mathbb{R}^{2}} \mathcal{E}(\widetilde{x}-\widetilde{y}, \widetilde{v}-\widetilde{w}) \tilde{f}(t, \widetilde{y}, \widetilde{w}) \mathrm{d} \tilde{w} \mathrm{~d} \tilde{y}, \quad(\widetilde{x}, \widetilde{v}) \in \mathbb{R}^{2} \times \mathbb{R}^{2}
$$

Therefore the effective trajectories satisfy the system

$$
\frac{\mathrm{d} \widetilde{X}}{\mathrm{~d} t}=\mathcal{V}[\tilde{f}(t)](\widetilde{X}(t), \widetilde{V}(t)), \quad \frac{\mathrm{d} \widetilde{V}}{\mathrm{~d} t}=\mathcal{A}[\tilde{f}(t)](\widetilde{X}(t), \widetilde{V}(t)), \quad(\widetilde{X}, \widetilde{V})(0 ; \widetilde{x}, \widetilde{v})=(\widetilde{x}, \widetilde{v})
$$

with

$$
\mathcal{V}[\tilde{f}(t)](\widetilde{x}, \widetilde{v})=-\omega_{c}^{-1}{ }^{\perp} \nabla_{\widetilde{x}} \tilde{\phi}[\tilde{f}(t)], \quad \mathcal{A}[\tilde{f}(t)](\widetilde{x}, \widetilde{v})=\omega_{c}{ }^{\perp} \nabla_{\widetilde{v}} \tilde{\phi}[\tilde{f}(t)], \quad(\widetilde{x}, \widetilde{v}) \in \mathbb{R}^{2} \times \mathbb{R}^{2}
$$




$$
\tilde{\phi}[\tilde{f}(t)](\widetilde{x}, \widetilde{v})=\int_{\mathbb{R}^{2}} \int_{\mathbb{R}^{2}} \mathcal{E}(\widetilde{x}-\widetilde{y}, \widetilde{v}-\widetilde{w}) \tilde{f}(t, \widetilde{y}, \widetilde{w}) \mathrm{d} \tilde{w} \mathrm{~d} \tilde{y}, \quad(\widetilde{x}, \widetilde{v}) \in \mathbb{R}^{2} \times \mathbb{R}^{2}
$$

and the conclusion of Theorem 1.1 follows formally, once that we justify (15). Before doing that, let us pay attention to the form of the characteristic system (21).

Proposition 2.1 The characteristic system (21) is Hamiltonian, with respect to the conjugate variables $\left(\widetilde{x}_{2}, \omega_{c}^{-1} \widetilde{v}_{1}\right)$ and $\left(\omega_{c} \widetilde{x}_{1}, \widetilde{v}_{2}\right)$ and the Hamiltonian function $\tilde{\phi}[\tilde{f}]$.

Proof. It is enough to observe that the equations in (21) write

$$
\begin{gathered}
\frac{\mathrm{d} \widetilde{X}_{2}}{\mathrm{~d} t}=\frac{\partial \tilde{\phi}[\tilde{f}(t)]}{\partial\left(\omega_{c} \widetilde{x}_{1}\right)}(\widetilde{X}(t), \widetilde{V}(t)), \quad \frac{\mathrm{d}\left(\omega_{c}^{-1} \widetilde{V}_{1}\right)}{\mathrm{d} t}=\frac{\partial \tilde{\phi}[\tilde{f}(t)]}{\partial \widetilde{v}_{2}}(\widetilde{X}(t), \widetilde{V}(t)) \\
\frac{\mathrm{d}\left(\omega_{c} \widetilde{X}_{1}\right)}{\mathrm{d} t}=-\frac{\partial \tilde{\phi}[\tilde{f}(t)]}{\partial \widetilde{x}_{2}}(\widetilde{X}(t), \widetilde{V}(t)), \quad \frac{\mathrm{d} \widetilde{V}_{2}}{\mathrm{~d} t}=-\frac{\partial \tilde{\phi}[\tilde{f}(t)]}{\partial\left(\omega_{c}^{-1} \widetilde{v}_{1}\right)}(\widetilde{X}(t), \widetilde{V}(t))
\end{gathered}
$$

saying that

$$
\frac{\mathrm{d}}{\mathrm{d} t}{ }^{t}\left(\widetilde{X}_{2}, \omega_{c}^{-1} \widetilde{V}_{1}\right)=\nabla_{\omega_{c} \widetilde{x}_{1}, \widetilde{v}_{2}} \tilde{\phi}[\tilde{f}(t)](\widetilde{X}(t), \widetilde{V}(t))
$$

and

$$
\frac{\mathrm{d}}{\mathrm{d} t}{ }^{t}\left(\omega_{c} \widetilde{X}_{1}, \widetilde{V}_{2}\right)=-\nabla_{\widetilde{x}_{2}, \omega_{c}^{-1} \widetilde{v}_{1}} \tilde{\phi}[\tilde{f}(t)](\widetilde{X}(t), \widetilde{V}(t)) .
$$

Let us come back to the computation of the function $\mathcal{E}(\xi, \eta)$. By the definition in $(20)$, the function $\mathcal{E}(\xi, \eta)$ is the average of the fundamental solution $e(\cdot)$ over the circle of center $\xi$ and radius $|\eta| /\left|\omega_{c}\right|$ and therefore we appeal to the mean property of the harmonic functions. At least in the case $|\xi|>|\eta| /\left|\omega_{c}\right|$, the function $z \rightarrow e(z)$ is harmonic in the open set $\mathbb{R}^{2} \backslash\{0\}$, which contains the disc $\left\{z \in \mathbb{R}^{2}:|z-\xi| \leq|\eta| /\left|\omega_{c}\right|\right\}$ and thus, the mean property applied to the function $e(\cdot)$ and the circle of center $\xi$ and radius $|\eta| /\left|\omega_{c}\right|$ yields

$$
\mathcal{E}(\xi, \eta)=\frac{1}{2 \pi} \int_{0}^{2 \pi} e\left(\xi-\frac{\mathcal{R}(\theta)}{\omega_{c}}{ }^{\perp} \eta\right) \mathrm{d} \theta=e(\xi)=-\frac{1}{2 \pi} \ln |\xi|, \quad|\xi|>\frac{|\eta|}{\left|\omega_{c}\right|} .
$$

More generally we prove the following proposition, see Appendix A for details.

Proposition 2.2 Let $\mathcal{E}: \mathbb{R}^{2} \times \mathbb{R}^{2} \backslash\{(0,0)\}$ be the function defined by

$$
\mathcal{E}(\xi, \eta)=\frac{1}{2 \pi} \int_{0}^{2 \pi} e\left(\xi-\frac{\mathcal{R}(\theta)}{\omega_{c}}{ }^{\perp} \eta\right) \mathrm{d} \theta, \quad(\xi, \eta) \in \mathbb{R}^{2} \times \mathbb{R}^{2} \backslash\{(0,0)\}
$$

where $e(z)=-\frac{1}{2 \pi} \ln |z|, z \in \mathbb{R}^{2} \backslash\{0\}$.

1. For any $(\xi, \eta) \in \mathbb{R}^{2} \times \mathbb{R}^{2} \backslash\{(0,0)\}$ we have

$$
\mathcal{E}(\xi, \eta)=e\left(\frac{\eta}{\omega_{c}}\right) \mathbf{1}_{\left\{|\xi| \leq|\eta| /\left|\omega_{c}\right|\right\}}+e(\xi) \mathbf{1}_{\left\{|\xi|>|\eta| /\left|\omega_{c}\right|\right\}} .
$$

In particular, $\mathcal{E}$ is locally integrable on $\mathbb{R}^{2} \times \mathbb{R}^{2}$. 
2. The first order partial derivatives of $\mathcal{E}$ write

$$
\nabla_{\xi} \mathcal{E}(\xi, \eta)=\nabla e(\xi) \mathbf{1}_{\left\{|\xi|>|\eta| /\left|\omega_{c}\right|\right\}}, \quad \nabla_{\eta} \mathcal{E}(\xi, \eta)=\omega_{c}^{-1} \nabla e\left(\frac{\eta}{\omega_{c}}\right) \mathbf{1}_{\left\{|\xi| \leq|\eta| /\left|\omega_{c}\right|\right\}} \text { in } \mathcal{D}^{\prime}\left(\mathbb{R}^{2} \times \mathbb{R}^{2}\right) .
$$

3. The second order partial derivatives of $\mathcal{E}$ write

$$
\begin{aligned}
\partial_{\xi}^{2} \mathcal{E}= & \left(I_{2}-2 \frac{\xi \otimes \xi}{|\xi|^{2}}\right) \frac{\mathbf{1}_{\left\{|\xi|>|\eta| /\left|\omega_{c}\right|\right\}} \mathrm{d}(\xi, \eta)}{2 \pi|\xi|^{2}}-\frac{\xi \otimes \xi}{|\xi|^{2}} \frac{\mathbf{1}_{\left\{|\xi|=|\eta| /\left|\omega_{c}\right|\right\}} \mathrm{d} \sigma(\xi, \eta)}{2 \pi|\xi| \sqrt{1+\omega_{c}^{-2}}} \\
& \left(\nabla_{\xi} \otimes \nabla_{\eta}\right) \mathcal{E}={ }^{t}\left(\nabla_{\eta} \otimes \nabla_{\xi}\right) \mathcal{E}=\frac{\xi \otimes \eta}{|\xi||\eta|} \frac{\mathbf{1}_{\left\{|\xi|=|\eta| /\left|\omega_{c}\right|\right\}} \mathrm{d} \sigma(\xi, \eta)}{2 \pi \omega_{c}|\eta| \sqrt{1+\omega_{c}^{-2}}} \\
\partial_{\eta}^{2} \mathcal{E}=- & \left(I_{2}-2 \frac{\eta \otimes \eta}{|\eta|^{2}}\right) \frac{\mathbf{1}_{\left\{|\xi| \leq|\eta| /\left|\omega_{c}\right|\right\}} \mathrm{d}(\xi, \eta)}{2 \pi|\eta|^{2}}-\frac{\eta \otimes \eta}{|\eta|^{2}} \frac{\mathbf{1}_{\left\{|\xi|=|\eta| /\left|\omega_{c}\right|\right\}} \mathrm{d} \sigma(\xi, \eta)}{2 \pi|\eta| \sqrt{1+\omega_{c}^{2}}} .
\end{aligned}
$$

In particular we have

$$
\Delta_{\xi} \mathcal{E}=-\frac{\mathbf{1}_{\left\{|\xi|=|\eta| /\left|\omega_{c}\right|\right\}} \mathrm{d} \sigma(\xi, \eta)}{2 \pi|\xi| \sqrt{1+\omega_{c}^{-2}}}, \Delta_{\eta} \mathcal{E}=-\frac{\mathbf{1}_{\left\{|\xi|=|\eta| /\left|\omega_{c}\right|\right\}} \mathrm{d} \sigma(\xi, \eta)}{2 \pi|\eta| \sqrt{1+\omega_{c}^{2}}} .
$$

Remark 2.1 The function $\mathcal{E}$ has also the symmetry property $\mathcal{E}\left(\omega_{c}^{-1} \eta, \omega_{c} \xi\right)=\mathcal{E}(\xi, \eta)$ for any $(\xi, \eta) \in\left(\mathbb{R}^{2} \times \mathbb{R}^{2}\right) \backslash\{(0,0)\}$. Indeed, we have by the first statement of Proposition 2.2

$$
\mathcal{E}\left(\frac{\eta}{\omega_{c}}, \omega_{c} \xi\right)=e(\xi) \mathbf{1}_{\left\{\frac{|\eta|}{\left|\omega_{c}\right|} \leq|\xi|\right\}}+e\left(\frac{\eta}{\omega_{c}}\right) \mathbf{1}_{\left\{\frac{|\eta|}{\left|\omega_{c}\right|}>|\xi|\right\}}=\mathcal{E}(\xi, \eta)
$$

\subsection{The properties of the effective Vlasov-Poisson system}

We investigate the main properties of the limit Vlasov-Poisson problem (12), (13). For simplicity we work with smooth densities $\tilde{f}$, compactly supported in the phase space $\mathbb{R}^{2} \times$ $\mathbb{R}^{2}$. Thanks to Proposition 2.2, we obtain the following expressions for the velocity and acceleration fields $\mathcal{V}[\tilde{f}], \mathcal{A}[\tilde{f}]$.

Proposition 2.3 The velocity and acceleration fields associated to any density $\tilde{f}=\tilde{f}(\widetilde{x}, \widetilde{v})$ write

$$
\begin{aligned}
& \mathcal{V}[\tilde{f}](\widetilde{x}, \widetilde{v})=\frac{1}{2 \pi \omega_{c}} \int_{\mathbb{R}^{2}} \int_{\mathbb{R}^{2}} \frac{\perp(\widetilde{x}-\widetilde{y})}{|\widetilde{x}-\widetilde{y}|^{2}} \tilde{f}(\widetilde{y}, \widetilde{w}) 1_{\left\{|\widetilde{x}-\widetilde{y}|>\frac{|\widetilde{v}-\widetilde{w}|}{\left|\omega_{c}\right|}\right.} \mathrm{d} \tilde{w} \mathrm{~d} \tilde{y} \\
& \mathcal{A}[\tilde{f}](\widetilde{x}, \widetilde{v})=-\frac{\omega_{c}}{2 \pi} \int_{\mathbb{R}^{2}} \int_{\mathbb{R}^{2}} \frac{\perp(\widetilde{v}-\widetilde{w})}{|\widetilde{f}-\widetilde{w}|^{2}} \tilde{f}(\widetilde{y}, \widetilde{w}) 1_{\left\{|\widetilde{x}-\widetilde{y}| \leq \frac{|\widetilde{v}-\widetilde{w}|}{\left|\omega_{c}\right|}\right\}} \mathrm{d} \tilde{w} \mathrm{~d} \tilde{y} .
\end{aligned}
$$

The Hamiltonian $\tilde{\phi}[\tilde{f}]$ verifies

$$
\begin{gathered}
-\Delta_{\widetilde{x}} \tilde{\phi}[\tilde{f}]=\frac{\left|\omega_{c}\right|}{2 \pi} \int_{\mathbb{R}^{2}} \frac{1}{|\widetilde{v}-\widetilde{w}|}\left(\int_{|\widetilde{x}-\widetilde{y}|=\frac{|\widetilde{v}-\widetilde{w}|}{\left|\omega_{c}\right|}} \tilde{f}(\widetilde{y}, \widetilde{w}) \mathrm{d} \sigma(\widetilde{y})\right) \mathrm{d} \tilde{w} \\
-\Delta_{\widetilde{v}} \tilde{\phi}[\tilde{f}]=\frac{1}{2 \pi\left|\omega_{c}\right|} \int_{\mathbb{R}^{2}} \frac{1}{|\widetilde{v}-\widetilde{w}|}\left(\int_{|\widetilde{x}-\widetilde{y}|=\frac{|\widetilde{v}-\widetilde{w}|}{\left|\omega_{c}\right|}} \tilde{f}(\widetilde{y}, \widetilde{w}) \mathrm{d} \sigma(\widetilde{y})\right) \mathrm{d} \tilde{w} .
\end{gathered}
$$


Proof. By the second statement of Proposition 2.2 we obtain

$$
\begin{aligned}
\nabla_{\widetilde{x}} \tilde{\phi}[\tilde{f}] & =\int_{\mathbb{R}^{2}} \int_{\mathbb{R}^{2}} \nabla_{\xi} \mathcal{E}(\widetilde{x}-\widetilde{y}, \widetilde{v}-\widetilde{w}) \tilde{f}(\widetilde{y}, \widetilde{w}) \mathrm{d} \tilde{w} \mathrm{~d} \tilde{y} \\
& =\int_{\mathbb{R}^{2}} \int_{\mathbb{R}^{2}} \nabla e(\widetilde{x}-\widetilde{y}) \mathbf{1}_{\left\{|\widetilde{x}-\widetilde{y}|>\frac{|\widetilde{v}-\widetilde{w}|}{\left|\omega_{c}\right|}\right.} \tilde{f}(\widetilde{y}, \widetilde{w}) \mathrm{d} \tilde{w} \mathrm{~d} \tilde{y}
\end{aligned}
$$

and

$$
\begin{aligned}
\nabla_{\widetilde{v}} \tilde{\phi}[\tilde{f}] & =\int_{\mathbb{R}^{2}} \int_{\mathbb{R}^{2}} \nabla_{\eta} \mathcal{E}(\widetilde{x}-\widetilde{y}, \widetilde{v}-\widetilde{w}) \tilde{f}(\widetilde{y}, \widetilde{w}) \mathrm{d} \tilde{w} \mathrm{~d} \tilde{y} \\
& =\int_{\mathbb{R}^{2}} \int_{\mathbb{R}^{2}} \frac{1}{\omega_{c}} \nabla e\left(\frac{\widetilde{v}-\widetilde{w}}{\omega_{c}}\right) \mathbf{1}_{\left\{|\widetilde{x}-\tilde{y}| \leq \frac{|\tilde{v}-\tilde{w}|}{\left|\omega_{c}\right|}\right\}} \tilde{f}(\widetilde{y}, \widetilde{w}) \mathrm{d} \tilde{w} \mathrm{~d} \tilde{y}
\end{aligned}
$$

which imply (22), (23). For the last two formulae we appeal to the last statement of Proposition 2.2. For any test function $\varphi \in C_{c}^{2}\left(\mathbb{R}^{2} \times \mathbb{R}^{2}\right)$ we deduce

$$
\begin{aligned}
\int_{\mathbb{R}^{2}} \int_{\mathbb{R}^{2}} \Delta_{\widetilde{x}} \varphi \tilde{\phi}[\tilde{f}](\widetilde{x}, \widetilde{v}) \mathrm{d} \tilde{v} \mathrm{~d} \tilde{x} & =\int_{\mathbb{R}^{2}} \int_{\mathbb{R}^{2}} \Delta_{\widetilde{x}} \varphi \int_{\mathbb{R}^{2}} \int_{\mathbb{R}^{2}} \mathcal{E}(\widetilde{x}-\widetilde{y}, \widetilde{v}-\widetilde{w}) \tilde{f}(\widetilde{y}, \widetilde{w}) \mathrm{d} \tilde{w} \mathrm{~d} \tilde{y} \mathrm{~d} \tilde{v} \mathrm{~d} \tilde{x} \\
& =\int_{\mathbb{R}^{2}} \int_{\mathbb{R}^{2}} \tilde{f}(\widetilde{y}, \widetilde{w}) \int_{\mathbb{R}^{2} \times \mathbb{R}^{2}} \Delta_{\tilde{x}} \varphi(\widetilde{y}+\xi, \widetilde{w}+\eta) \mathcal{E}(\xi, \eta) \mathrm{d}(\xi, \eta) \mathrm{d} \tilde{w} \mathrm{~d} \tilde{y} \\
& =-\int_{\mathbb{R}^{2}} \int_{\mathbb{R}^{2}} \tilde{f}(\widetilde{y}, \widetilde{w}) \int_{|\xi|=\frac{|\eta|}{\left|\omega_{c}\right|}} \frac{\varphi(\widetilde{y}+\xi, \widetilde{w}+\eta)}{2 \pi|\xi| \sqrt{1+\omega_{c}^{-2}} \mathrm{~d} \sigma(\xi, \eta) \mathrm{d} \tilde{w} \mathrm{~d} \tilde{y}} \\
& =-\int_{\mathbb{R}^{2}} \int_{\mathbb{R}^{2}} \tilde{f}(\widetilde{y}, \widetilde{w}) \int_{|\widetilde{x}-\widetilde{y}|=\frac{|\widetilde{v}-\widetilde{w}|}{\left|\omega_{c}\right|}} \frac{\varphi(\widetilde{x}, \widetilde{v})}{2 \pi|\widetilde{x}-\widetilde{y}| \sqrt{1+\omega_{c}^{-2}}} \mathrm{~d} \sigma(\widetilde{x}, \widetilde{v}) \mathrm{d} \tilde{w} \mathrm{~d} \tilde{y} \\
& =-\int_{\mathbb{R}^{2}} \int_{\mathbb{R}^{2}} \varphi(\widetilde{x}, \widetilde{v}) \int_{|\widetilde{x}-\widetilde{y}|=\frac{|\widetilde{v}-\widetilde{w}|}{\left|\omega_{c}\right|}} \frac{\tilde{f}(\widetilde{w})}{2 \pi|\widetilde{x}-\widetilde{y}| \sqrt{1+\omega_{c}^{-2}}} \mathrm{~d} \sigma(\widetilde{y}, \widetilde{w}) \mathrm{d} \tilde{v} \mathrm{~d} \tilde{x}
\end{aligned}
$$

and therefore $\tilde{\phi}[\tilde{f}]$ satisfies

$$
\begin{aligned}
-\Delta_{\widetilde{x}} \tilde{\phi}[\tilde{f}] & =\int_{|\widetilde{x}-\widetilde{y}|=\frac{|\widetilde{v}-\widetilde{w}|}{\left|\omega_{c}\right|}} \frac{\tilde{f}(\widetilde{y}, \widetilde{w})}{2 \pi|\widetilde{x}-\widetilde{y}| \sqrt{1+\omega_{c}^{-2}}} \mathrm{~d} \sigma(\widetilde{y}, \widetilde{w}) \\
& =\frac{\left|\omega_{c}\right|}{2 \pi} \int_{\mathbb{R}^{2}} \frac{1}{|\widetilde{v}-\widetilde{w}|}\left(\int_{|\widetilde{x}-\widetilde{y}|=\frac{|\widetilde{v}-\widetilde{w}|}{\left|\omega_{c}\right|}} \tilde{f}(\widetilde{y}, \widetilde{w}) \mathrm{d} \sigma(\widetilde{y})\right) \mathrm{d} \widetilde{w} .
\end{aligned}
$$

Following the same lines, we obtain

$$
\begin{aligned}
\int_{\mathbb{R}^{2}} \int_{\mathbb{R}^{2}} \Delta_{\widetilde{v}} \varphi \tilde{\phi}[\tilde{f}](\widetilde{x}, \widetilde{v}) \mathrm{d} \tilde{v} \mathrm{~d} \tilde{x} & =\int_{\mathbb{R}^{2}} \int_{\mathbb{R}^{2}} \Delta_{\widetilde{v}} \varphi \int_{\mathbb{R}^{2}} \int_{\mathbb{R}^{2}} \mathcal{E}(\widetilde{x}-\widetilde{y}, \widetilde{v}-\widetilde{w}) \tilde{f}(\widetilde{y}, \widetilde{w}) \mathrm{d} \tilde{w} \mathrm{~d} \tilde{y} \mathrm{~d} \tilde{v} \mathrm{~d} \tilde{x} \\
& =\int_{\mathbb{R}^{2}} \int_{\mathbb{R}^{2}} \tilde{f}(\widetilde{y}, \widetilde{w}) \int_{\mathbb{R}^{2} \times \mathbb{R}^{2}} \Delta_{\widetilde{v}} \varphi(\widetilde{y}+\xi, \widetilde{w}+\eta) \mathcal{E}(\xi, \eta) \mathrm{d}(\xi, \eta) \mathrm{d} \tilde{w} \mathrm{~d} \tilde{y} \\
& =-\int_{\mathbb{R}^{2}} \int_{\mathbb{R}^{2}} \tilde{f}(\widetilde{y}, \widetilde{w}) \int_{|\xi|=\frac{|\eta|}{\left|\omega_{c}\right|}} \frac{\varphi(\widetilde{y}+\xi, \widetilde{w}+\eta)}{2 \pi|\eta| \sqrt{1+\omega_{c}^{2}}} \mathrm{~d} \sigma(\xi, \eta) \mathrm{d} \tilde{w} \mathrm{~d} \tilde{y} \\
& =-\int_{\mathbb{R}^{2}} \int_{\mathbb{R}^{2}} \tilde{f}(\widetilde{y}, \widetilde{w}) \int_{|\widetilde{x}-\widetilde{y}|=\frac{|\widetilde{v}-\widetilde{w}|}{\left|\omega_{c}\right|}} \frac{\varphi(\widetilde{x}, \widetilde{v})}{2 \pi|\widetilde{v}-\widetilde{w}| \sqrt{1+\omega_{c}^{2}}} \mathrm{~d} \sigma(\widetilde{x}, \widetilde{v}) \mathrm{d} \tilde{w} \mathrm{~d} \tilde{y} \\
& =-\int_{\mathbb{R}^{2}} \int_{\mathbb{R}^{2}} \varphi(\widetilde{x}, \widetilde{v}) \int_{|\widetilde{x}-\widetilde{y}|=\frac{|\widetilde{v}-\widetilde{v}|}{\left|\omega_{c}\right|}} \frac{\tilde{f}(\widetilde{w})}{2 \pi|\widetilde{v}-\widetilde{w}| \sqrt{1+\omega_{c}^{2}}} \mathrm{~d} \sigma(\widetilde{y}, \widetilde{w}) \mathrm{d} \tilde{v} \mathrm{~d} \tilde{x}
\end{aligned}
$$


which says

$$
\begin{aligned}
-\Delta_{\widetilde{v}} \tilde{\phi}[\tilde{f}] & =\frac{1}{\left|\omega_{c}\right|} \int_{|\widetilde{x}-\widetilde{y}|=\frac{|\widetilde{v}-\widetilde{w}|}{\left|\omega_{c}\right|}} \frac{\tilde{f}(\widetilde{y}, \widetilde{w})}{2 \pi|\widetilde{v}-\widetilde{w}| \sqrt{1+\omega_{c}^{-2}}} \mathrm{~d} \sigma(\widetilde{y}, \widetilde{w}) \\
& =\frac{1}{2 \pi\left|\omega_{c}\right|} \int_{\mathbb{R}^{2}} \frac{1}{|\widetilde{v}-\widetilde{w}|}\left(\int_{|\widetilde{x}-\widetilde{y}|=\frac{|\widetilde{v}-\widetilde{w}|}{\left|\omega_{c}\right|}} \tilde{f}(\widetilde{y}, \widetilde{w}) \mathrm{d} \sigma(\widetilde{y})\right) \mathrm{d} \widetilde{w} .
\end{aligned}
$$

Clearly the advection field $(\mathcal{V}[\tilde{f}], \mathcal{A}[\tilde{f}])$ is divergence free. Moreover, other divergence constraints hold true. They are direct consequences of Proposition 2.3 and are summarized below.

Corollary 2.1 The velocity and acceleration fields associated to any density $\tilde{f}=\tilde{f}(\widetilde{x}, \widetilde{v})$ satisfy

$$
\begin{gathered}
\operatorname{div}_{\tilde{x}} \mathcal{V}[\tilde{f}]=0, \quad \operatorname{div}_{\widetilde{v}} \mathcal{A}[\tilde{f}]=0 \\
\operatorname{div}_{\widetilde{x}}{ }^{\perp} \mathcal{V}[\tilde{f}]+\operatorname{div}_{\widetilde{v}}{ }^{\perp} \mathcal{A}[\tilde{f}]=0 \\
\operatorname{div}_{\widetilde{x}} \mathcal{A}[\tilde{f}]-\omega_{c}^{2} \operatorname{div}_{\widetilde{v}} \mathcal{V}[\tilde{f}]=0 \\
\operatorname{div}_{\widetilde{x}}{ }^{\perp} \mathcal{A}[\tilde{f}]+\omega_{c}^{2} \operatorname{div}_{\widetilde{v}}{ }^{\perp} \mathcal{V}[\tilde{f}]=0 .
\end{gathered}
$$

Proof. The equalities in (26) come immediately by the relations

$$
\mathcal{V}[\tilde{f}]=-\frac{{ }^{\perp} \nabla_{\tilde{x}} \tilde{\phi}[\tilde{f}]}{\omega_{c}}, \mathcal{A}[\tilde{f}]=\omega_{c}{ }^{\perp} \nabla_{\widetilde{v}} \tilde{\phi}[\tilde{f}] .
$$

The statement in (27) is a consequence of $(24),(25)$

$$
\operatorname{div}_{\widetilde{x}}{ }^{\perp} \mathcal{V}[\tilde{f}]+\operatorname{div}_{\widetilde{v}}{ }^{\perp} \mathcal{A}[\tilde{f}]=\operatorname{div}_{\widetilde{x}} \frac{\nabla_{\widetilde{x}} \tilde{\phi}[\tilde{f}]}{\omega_{c}}-\omega_{c} \operatorname{div}_{\widetilde{v}} \nabla_{\widetilde{v}} \tilde{\phi}[\tilde{f}]=\frac{\Delta_{\widetilde{x}} \tilde{\phi}[\tilde{f}]}{\omega_{c}}-\omega_{c} \Delta_{\widetilde{v}} \tilde{\phi}[\tilde{f}]=0
$$

For the last two statements observe that

$$
\operatorname{div}_{\widetilde{x}} \mathcal{A}[\tilde{f}]=\omega_{c} \operatorname{div}_{\widetilde{x}}\left({ }^{\perp} \nabla_{\widetilde{v}} \tilde{\phi}[\tilde{f}]\right)=-\omega_{c} \operatorname{div}_{\widetilde{v}}\left({ }^{\perp} \nabla_{\widetilde{x}} \tilde{\phi}[\tilde{f}]\right)=\omega_{c}^{2} \operatorname{div}_{\widetilde{v}} \mathcal{V}[\tilde{f}]
$$

and

$$
\operatorname{div}_{\widetilde{x}}{ }^{\perp} \mathcal{A}[\tilde{f}]=-\omega_{c} \operatorname{div}_{\widetilde{x}}\left(\nabla_{\widetilde{v}} \tilde{\phi}[\tilde{f}]\right)=-\omega_{c} \operatorname{div}_{\widetilde{v}}\left(\nabla_{\widetilde{x}} \tilde{\phi}[\tilde{f}]\right)=-\omega_{c}^{2} \operatorname{div}_{\widetilde{v}}\left({ }^{\perp} \mathcal{V}[\tilde{f}]\right)
$$

We inquire now about the conservations of the limit model (12), (13). The velocity and acceleration fields being divergence free, the equation (12) writes also into conservative form

$$
\partial_{t} \tilde{f}+\operatorname{div}_{\tilde{x}}\{\tilde{f} \mathcal{V}[\tilde{f}]\}+\operatorname{div}_{\widetilde{v}}\{\tilde{f} \mathcal{A}[\tilde{f}]\}=0, \quad(t, \widetilde{x}, \widetilde{v}) \in \mathbb{R}_{+} \times \mathbb{R}^{2} \times \mathbb{R}^{2}
$$

which implies the mass conservation. We search for other moments of $\tilde{f}$ which are conserved in time. We establish first the following lemma. 
Lemma 2.1 Let $\tilde{f}=\tilde{f}(t, \widetilde{x}, \widetilde{v})$ be a solution of (12), (14), (15) and $\psi=\psi(\widetilde{x}, \widetilde{v})$ be a $C^{1}$ function. For any $t \in \mathbb{R}_{+}$we have

$$
\begin{aligned}
& 2 \frac{\mathrm{d}}{\mathrm{d} t} \int_{\mathbb{R}^{2}} \int_{\mathbb{R}^{2}} \psi(\widetilde{x}, \widetilde{v}) \tilde{f}(t, \widetilde{x}, \widetilde{v}) \mathrm{d} \tilde{v} \mathrm{~d} \tilde{x}=\int_{\mathbb{R}^{2}} \int_{\mathbb{R}^{2}} \int_{\mathbb{R}^{2}} \int_{\mathbb{R}^{2}} \tilde{f}(t, \widetilde{y}, \widetilde{w}) \tilde{f}(t, \widetilde{x}, \widetilde{v}) \\
& \times\left[\frac{1}{\omega_{c}}\left(\nabla_{\widetilde{y}} \psi(\widetilde{y}, \widetilde{w})-\nabla_{\widetilde{x}} \psi(\widetilde{x}, \widetilde{v})\right) \cdot{ }^{\perp} \nabla e(\widetilde{x}-\widetilde{y}) \mathbf{1}_{\left\{|\widetilde{x}-\widetilde{y}|>|\widetilde{v}-\widetilde{w}| /\left|\omega_{c}\right|\right\}}\right. \\
& +\left(\nabla_{\widetilde{v}} \psi(\widetilde{x}, \widetilde{v})-\nabla_{\widetilde{w}} \psi(\widetilde{y}, \widetilde{w})\right) \cdot{ }^{\perp} \nabla e\left(\frac{\widetilde{v}-\widetilde{w}}{\omega_{c}}\right) \mathbf{1}_{\left.\left\{|\widetilde{x}-\widetilde{y}|<|\widetilde{v}-\widetilde{w}| /\left|\omega_{c}\right|\right\}\right] \mathrm{d} \widetilde{w} \mathrm{~d} \widetilde{y} \mathrm{~d} \widetilde{v} \mathrm{~d} \widetilde{x}}
\end{aligned}
$$

where $e(z)=-\frac{1}{2 \pi} \ln |z|, z \in \mathbb{R}^{2} \backslash\{0\}$ is the fundamental solution of the Laplace operator in $\mathbb{R}^{2}$.

Proof. We combine (12) with the representation formulae (22), (23). We obtain

$$
\begin{aligned}
& \frac{\mathrm{d}}{\mathrm{d} t} \int_{\mathbb{R}^{2}} \int_{\mathbb{R}^{2}} \psi(\widetilde{x}, \widetilde{v}) \tilde{f}(t, \widetilde{x}, \widetilde{v}) \mathrm{d} \tilde{v} \mathrm{~d} \tilde{x}=\int_{\mathbb{R}^{2}} \int_{\mathbb{R}^{2}}\left[\nabla_{\widetilde{x}} \psi \cdot \mathcal{V}[\tilde{f}(t)]+\nabla_{\widetilde{v}} \psi \cdot \mathcal{A}[\tilde{f}(t)]\right] \tilde{f}(t, \widetilde{x}, \widetilde{v}) \mathrm{d} \tilde{v} \mathrm{~d} \tilde{x} \\
& \quad=-\frac{1}{\omega_{c}} \int_{\mathbb{R}^{2}} \int_{\mathbb{R}^{2}} \int_{\mathbb{R}^{2}} \int_{\mathbb{R}^{2}} \nabla_{\widetilde{x}} \psi(\widetilde{x}, \widetilde{v}) \cdot{ }^{\perp} \nabla e(\widetilde{x}-\widetilde{y}) \mathbf{1}_{\left\{|\widetilde{x}-\widetilde{y}|>|\widetilde{v}-\widetilde{w}| /\left|\omega_{c}\right|\right\} \tilde{f}(t, \widetilde{y}, \widetilde{w}) \tilde{f}(t, \widetilde{x}, \widetilde{v}) \mathrm{d} \widetilde{w} \mathrm{~d} \widetilde{y} \mathrm{~d} \widetilde{v} \mathrm{~d} \widetilde{x}} \\
& \quad+\int_{\mathbb{R}^{2}} \int_{\mathbb{R}^{2}} \int_{\mathbb{R}^{2}} \int_{\mathbb{R}^{2}} \nabla_{\widetilde{v}} \psi(\widetilde{x}, \widetilde{v}) \cdot{ }^{\perp} \nabla e\left(\frac{\widetilde{v}-\widetilde{w}}{\omega_{c}}\right) \mathbf{1}_{\left\{|\widetilde{x}-\widetilde{y}|<|\widetilde{v}-\widetilde{w}| /\left|\omega_{c}\right|\right\}} \tilde{f}(t, \widetilde{y}, \widetilde{w}) \tilde{f}(t, \widetilde{x}, \widetilde{v}) \mathrm{d} \widetilde{w} \mathrm{~d} \widetilde{y} \mathrm{~d} \widetilde{v} \mathrm{~d} \widetilde{x}
\end{aligned}
$$

The key point is to interchange $(\widetilde{x}, \widetilde{v})$ against $(\widetilde{y}, \widetilde{w})$ and to apply Fubini theorem

$$
\begin{aligned}
& \frac{\mathrm{d}}{\mathrm{d} t} \int_{\mathbb{R}^{2}} \int_{\mathbb{R}^{2}} \psi(\widetilde{x}, \widetilde{v}) \tilde{f}(t, \widetilde{x}, \widetilde{v}) \mathrm{d} \tilde{v} \mathrm{~d} \tilde{x}=\int_{\mathbb{R}^{2}} \int_{\mathbb{R}^{2}} \int_{\mathbb{R}^{2}} \int_{\mathbb{R}^{2}} \tilde{f}(t, \widetilde{x}, \widetilde{v}) \tilde{f}(t, \widetilde{y}, \widetilde{w}) \mathrm{d} \tilde{w} \mathrm{~d} \tilde{y} \mathrm{~d} \tilde{v} \mathrm{~d} \tilde{x} \\
& \times\left[\frac{1}{\omega_{c}} \nabla_{\widetilde{y}} \psi(\widetilde{y}, \widetilde{w}) \cdot{ }^{\perp} \nabla e(\widetilde{x}-\widetilde{y}) \mathbf{1}_{\left\{|\widetilde{x}-\widetilde{y}|>\frac{|\widetilde{v}-\widetilde{w}|}{\left|\omega_{c}\right|}\right.}-\nabla_{\widetilde{w}} \psi(\widetilde{y}, \widetilde{w}) \cdot{ }^{\perp} \nabla e\left(\frac{\widetilde{v}-\widetilde{w}}{\omega_{c}}\right) \mathbf{1}_{\left\{|\widetilde{x}-\widetilde{y}|<\frac{|\widetilde{v}-\widetilde{w}|}{\left|\omega_{c}\right|}\right] .} .\right.
\end{aligned}
$$

Our conclusion follows by taking the sum of (30), (31).

The conclusions in Proposition 1.1 come easily thanks to Lemma 2.1.

Proof. (of Proposition 1.1)

1. The conservations of the limit Vlasov-Poisson problem follow immediately, taking as test function $1, \widetilde{x}, \widetilde{v},|\widetilde{x}|^{2},|\widetilde{v}|^{2}$.

2. The electric energy writes

$\frac{1}{2} \int_{\mathbb{R}^{2}} \int_{\mathbb{R}^{2}} \tilde{\phi}[\tilde{f}(t)](\widetilde{x}, \widetilde{v}) \tilde{f}(t, \widetilde{x}, \widetilde{v}) \mathrm{d} \tilde{v} \mathrm{~d} \tilde{x}=\frac{1}{2} \int_{\mathbb{R}^{2}} \int_{\mathbb{R}^{2}} \int_{\mathbb{R}^{2}} \int_{\mathbb{R}^{2}} \mathcal{E}(\widetilde{x}-\widetilde{y}, \widetilde{v}-\widetilde{w}) \tilde{f}(t, \widetilde{x}, \widetilde{v}) \tilde{f}(t, \widetilde{y}, \widetilde{w}) \mathrm{d} \tilde{w} \mathrm{~d} \tilde{y} \mathrm{~d} \tilde{v} \mathrm{~d} \tilde{x}$.

This quantity is non negative, cf. Proposition 3.4. Taking into account that $\mathcal{E}$ is even with respect to both variables (actually $\mathcal{E}(\xi, \eta)$ depends only on $|\xi|,|\eta|$ ), we obtain by Fubini Theorem

$$
\begin{aligned}
\frac{\mathrm{d}}{\mathrm{d} t} \frac{1}{2} \int_{\mathbb{R}^{2}} \int_{\mathbb{R}^{2}} \tilde{\phi} & {[\tilde{f}(t)](\widetilde{x}, \widetilde{v}) \tilde{f}(t, \widetilde{x}, \widetilde{v}) \mathrm{d} \tilde{v} \mathrm{~d} \tilde{x}=\int_{\mathbb{R}^{2}} \int_{\mathbb{R}^{2}} \tilde{\phi}[\tilde{f}(t)](\widetilde{x}, \widetilde{v}) \partial_{t} \tilde{f} \mathrm{~d} \tilde{v} \mathrm{~d} \tilde{x} } \\
& =\int_{\mathbb{R}^{2}} \int_{\mathbb{R}^{2}}\left[\nabla_{\tilde{x}} \tilde{\phi}[\tilde{f}(t)] \cdot \mathcal{V}[\tilde{f}(t)]+\nabla_{\widetilde{v}} \tilde{\phi}[\tilde{f}(t)] \cdot \mathcal{A}[\tilde{f}(t)]\right] \tilde{f} \mathrm{~d} \tilde{v} \mathrm{~d} \tilde{x}=0
\end{aligned}
$$

and therefore the electric energy is conserved. 


\section{Convergence results}

This section is devoted to the rigorous justification of the asymptotic behavior as $\varepsilon \searrow 0$ for the family $\left(f^{\varepsilon}, \phi^{\varepsilon}\right)_{\varepsilon>0}$ formally derived in Section 2. We make the following hypotheses on the initial density $f^{\text {in }}(x, v)$

$\mathrm{H} 1 \quad \int_{\mathbb{R}^{2}} \int_{\mathbb{R}^{2}} f^{\text {in }}(x, v) \mathrm{d} v \mathrm{~d} x<+\infty$

$\mathrm{H} 2 \quad \int_{\mathbb{R}^{2}} \int_{\mathbb{R}^{2}} \frac{|v|^{2}}{2} f^{\mathrm{in}}(x, v) \mathrm{d} v \mathrm{~d} x<+\infty$

H3 there is a bounded, non increasing function $F^{\text {in }}=F^{\text {in }}(r) \in L^{\infty} \cap L^{1}\left(\mathbb{R}_{+} ; r \mathrm{~d} r\right)$, such that $f^{\text {in }}(x, v) \leq F^{\text {in }}(|v|), \quad(x, v) \in \mathbb{R}^{2} \times \mathbb{R}^{2}$.

Under the above assumptions, it is well known that for any $\varepsilon>0$, there is a weak solution for the Vlasov-Poisson problem (9), (10), (11) cf. [2, 14, 4]. Following the arguments in [4], leads to a $L^{\infty}$ estimate for the electric field, and therefore, if the initial density $f^{\text {in }}$ is smooth, with compact support, then so is the restriction of $f^{\varepsilon}$ on $[0, T] \times \mathbb{R}^{2} \times \mathbb{R}^{2}$ for any $T \in \mathbb{R}_{+}$, see [12]. We recall briefly the a priori estimates for the solutions $\left(f^{\varepsilon}, \phi^{\varepsilon}\right)_{\varepsilon>0}$. The continuity equation

$$
\partial_{t} \rho^{\varepsilon}+\frac{1}{\varepsilon} \operatorname{div}_{x} j^{\varepsilon}=0, \quad \rho^{\varepsilon}=\int_{\mathbb{R}^{2}} f^{\varepsilon} \mathrm{d} v, \quad j^{\varepsilon}=\int_{\mathbb{R}^{2}} v f^{\varepsilon} \mathrm{d} v
$$

implies the total mass conservation

$$
\int_{\mathbb{R}^{2}} \int_{\mathbb{R}^{2}} f^{\varepsilon}(t, x, v) \mathrm{d} v \mathrm{~d} x=\int_{\mathbb{R}^{2}} \int_{\mathbb{R}^{2}} f^{\mathrm{in}}(x, v) \mathrm{d} v \mathrm{~d} x, \quad t \in \mathbb{R}_{+} .
$$

By standard computations we obtain the conservation of the total energy

$$
\frac{\mathrm{d}}{\mathrm{d} t}\left(\int_{\mathbb{R}^{2}} \int_{\mathbb{R}^{2}} \frac{|v|^{2}}{2} f^{\varepsilon}(t, x, v) \mathrm{d} v \mathrm{~d} x+\frac{\varepsilon}{2} \int_{\mathbb{R}^{2}}\left|\nabla_{x} \phi^{\varepsilon}(t, x)\right|^{2} \mathrm{~d} x\right)=0, \quad t \in \mathbb{R}_{+} .
$$

Usual interpolation inequalities provide an estimate for the $L^{2}$ norm of the charge densities $\left(\rho^{\varepsilon}\right)_{\varepsilon>0}$

$$
\left\|\rho^{\varepsilon}(t)\right\|_{L^{2}\left(\mathbb{R}^{2}\right)} \leq 2 \sqrt{\pi}\left\|f^{\varepsilon}\right\|_{L^{\infty}}^{1 / 2}\left(\int_{\mathbb{R}^{2}} \int_{\mathbb{R}^{2}}|v|^{2} f^{\varepsilon}(x, v) \mathrm{d} v \mathrm{~d} x\right)^{1 / 2}
$$

In particular, thanks to $\mathrm{H} 2$, $\mathrm{H} 3$, we have

$$
\begin{aligned}
\left\|\rho^{\mathrm{in}}\right\|_{L^{2}\left(\mathbb{R}^{2}\right)} & \leq 2 \sqrt{\pi}\left\|f^{\mathrm{in}}\right\|_{L^{\infty}}^{1 / 2}\left(\int_{\mathbb{R}^{2}} \int_{\mathbb{R}^{2}}|v|^{2} f^{\mathrm{in}}(x, v) \mathrm{d} v \mathrm{~d} x\right)^{1 / 2} \\
& \leq 2 \sqrt{\pi}\left\|F^{\mathrm{in}}\right\|_{L^{\infty}}\left(\int_{\mathbb{R}^{2}} \int_{\mathbb{R}^{2}}|v|^{2} f^{\mathrm{in}}(x, v) \mathrm{d} v \mathrm{~d} x\right)^{1 / 2}<+\infty
\end{aligned}
$$


implying that $E^{\varepsilon}(0)=-\nabla_{x} \phi^{\varepsilon}(0)$ belongs to $H^{1}\left(\mathbb{R}^{2}\right)$. Therefore, by the energy conservation (32) we deduce

$$
\sup _{0<\varepsilon<1, t \in \mathbb{R}_{+}}\left(\int_{\mathbb{R}^{2}} \int_{\mathbb{R}^{2}} \frac{|v|^{2}}{2} f^{\varepsilon}(t, x, v) \mathrm{d} v \mathrm{~d} x+\int_{\mathbb{R}^{2}}\left(\rho^{\varepsilon}(t, x)\right)^{2} \mathrm{~d} x\right)<+\infty
$$

and

$$
\sup _{0<\varepsilon<1, t \in \mathbb{R}_{+}}\left\|\nabla_{x} \phi^{\varepsilon}(t)\right\|_{H^{1}\left(\mathbb{R}^{2}\right)}<+\infty .
$$

Moreover, uniform $L^{\infty}$ bounds are available for the charge densities $\rho^{\varepsilon}$ and the electric fields $E^{\varepsilon}:=-\nabla_{x} \phi^{\varepsilon}$. For any $(t, x) \in \mathbb{R}_{+} \times \mathbb{R}^{2}$ and $R>0$ we write

$$
\begin{aligned}
\left|\nabla_{x} \phi^{\varepsilon}(t, x)\right| & =\left|\int_{\mathbb{R}^{2}} \nabla e(x-y) \rho^{\varepsilon}(t, y) \mathrm{d} y\right| \leq \frac{1}{2 \pi} \int_{|x-y|<R} \frac{\rho^{\varepsilon}(t, y)}{|x-y|} \mathrm{d} y+\frac{1}{2 \pi} \int_{|x-y| \geq R} \frac{\rho^{\varepsilon}(t, y)}{|x-y|} \mathrm{d} y \\
& \leq R\left\|\rho^{\varepsilon}(t)\right\|_{L^{\infty}\left(\mathbb{R}^{2}\right)}+\frac{1}{2 \pi R}\left\|\rho^{\varepsilon}(t)\right\|_{L^{1}\left(\mathbb{R}^{2}\right)} .
\end{aligned}
$$

Therefore, minimizing with respect to $R>0$, we obtain the inequality

$$
\left\|\nabla_{x} \phi^{\varepsilon}(t)\right\|_{L^{\infty}\left(\mathbb{R}^{2}\right)} \leq \sqrt{\frac{2}{\pi}}\left\|\rho^{\varepsilon}(t)\right\|_{L^{\infty}\left(\mathbb{R}^{2}\right)}^{1 / 2}\left\|f^{\mathrm{in}}\right\|_{L^{1}\left(\mathbb{R}^{2} \times \mathbb{R}^{2}\right)}^{1 /}
$$

For estimating the charge density $\rho^{\varepsilon}$, observe that $f^{\varepsilon}(t, x, v)=f^{\text {in }}\left(X^{\varepsilon}(0 ; t, x, v), V^{\varepsilon}(0 ; t, x, v)\right)$ where $\left(X^{\varepsilon}, V^{\varepsilon}\right)$ solve the characteristic system

$$
\frac{\mathrm{d} X^{\varepsilon}}{\mathrm{d} t}=\frac{V^{\varepsilon}(t)}{\varepsilon}, \frac{\mathrm{d} V^{\varepsilon}}{\mathrm{d} t}=E^{\varepsilon}\left(t, X^{\varepsilon}(t)\right)+\frac{\omega_{c}}{\varepsilon}{ }^{\perp} V^{\varepsilon}(t)
$$

with the condition $\left(X^{\varepsilon}, V^{\varepsilon}\right)(t ; t, x, v)=(x, v)$. It is easily seen that

$$
\left|\frac{\mathrm{d}}{\mathrm{d} t}\right| V^{\varepsilon}(t)|| \leq\left|E^{\varepsilon}\left(t, X^{\varepsilon}(t)\right)\right| \leq\left\|E^{\varepsilon}(t)\right\|_{L^{\infty}\left(\mathbb{R}^{2}\right)}
$$

implying that

$$
\left|V^{\varepsilon}(0 ; \tau, x, v)\right| \geq|v|-R^{\varepsilon}(t), \quad \tau \in[0, t], \quad R^{\varepsilon}(t)=\int_{0}^{t}\left\|E^{\varepsilon}(\tau)\right\|_{L^{\infty}} \mathrm{d} \tau .
$$

The hypothesis H3 yields for any $t \in \mathbb{R}_{+}$

$$
f^{\varepsilon}(t, x, v)=f^{\text {in }}\left(X^{\varepsilon}(0 ; t, x, v), V^{\varepsilon}(0 ; t, x, v)\right) \leq F^{\text {in }}\left(\left|V^{\varepsilon}(0 ; t, x, v)\right|\right)
$$

and therefore

$$
\begin{aligned}
\rho^{\varepsilon}(t, x) & \leq \int_{\mathbb{R}^{2}} F^{\mathrm{in}}\left(\left|V^{\varepsilon}(0 ; t, x, v)\right|\right) \mathrm{d} v \leq \int_{|v|<2 R^{\varepsilon}(t)} F^{\mathrm{in}}(0) \mathrm{d} v+\int_{|v| \geq 2 R^{\varepsilon}(t)} F^{\mathrm{in}}\left(|v|-R^{\varepsilon}(t)\right) \mathrm{d} v \\
& \leq 4 \pi\left(R^{\varepsilon}(t)\right)^{2}\left\|F^{\mathrm{in}}\right\|_{L^{\infty}}+4 \pi\left\|F^{\mathrm{in}}\right\|_{L^{1}\left(\mathbb{R}_{+} ; r \mathrm{~d} r\right) .}
\end{aligned}
$$


Combining (33) and (34) leads to

$$
\begin{aligned}
\left\|E^{\varepsilon}(t)\right\|_{L^{\infty}} & \leq \sqrt{\frac{2}{\pi}}\left\|f^{\mathrm{in}}\right\|_{L^{1}\left(\mathbb{R}^{2} \times \mathbb{R}^{2}\right)}^{1 / 2} 2 \sqrt{\pi}\left(\left(R^{\varepsilon}(t)\right)^{2}\left\|F^{\mathrm{in}}\right\|_{L^{\infty}}+\left\|F^{\mathrm{in}}\right\|_{L^{1}\left(\mathbb{R}_{+} ; r \mathrm{~d} r\right)}\right)^{1 / 2} \\
& \leq 2 \sqrt{2}\left\|f^{\mathrm{in}}\right\|_{L^{1}\left(\mathbb{R}^{2} \times \mathbb{R}^{2}\right)}^{1 / 2}\left(\left\|F^{\mathrm{in}}\right\|_{L^{1}\left(\mathbb{R}_{+} ; r \mathrm{~d} r\right)}^{1 / 2}+\left\|F^{\mathrm{in}}\right\|_{L^{\infty}}^{1 / 2} \int_{0}^{t}\left\|E^{\varepsilon}(\tau)\right\| \mathrm{d} \tau\right)
\end{aligned}
$$

and by Gronwall lemma we deduce that

$$
\sup _{\varepsilon>0}\left\|E^{\varepsilon}\right\|_{L^{\infty}\left([0, t] \times \mathbb{R}^{2}\right)}+\sup _{\varepsilon>0}\left\|\rho^{\varepsilon}\right\|_{L^{\infty}\left([0, t] \times \mathbb{R}^{2}\right)}<+\infty, \quad t \in \mathbb{R}_{+} .
$$

These conclusions are summarized up in the following proposition.

Proposition 3.1 Assume that $f^{\text {in }}$ satisfies the hypotheses H1, H2, H3. Let $\left(f^{\varepsilon}, \phi^{\varepsilon}\right)_{\varepsilon>0}$ be the solutions for the Vlasov-Poisson problems (9), (10), (11). Then the densities $f^{\varepsilon}$ are non negative and we have

$$
\begin{gathered}
\int_{\mathbb{R}^{2}} \int_{\mathbb{R}^{2}} f^{\varepsilon}(t) \mathrm{d} v \mathrm{~d} x=\int_{\mathbb{R}^{2}} \int_{\mathbb{R}^{2}} f^{\text {in }} \mathrm{d} v \mathrm{~d} x, \quad \int_{\mathbb{R}^{2}} \int_{\mathbb{R}^{2}}\left(f^{\varepsilon}(t)\right)^{2} \mathrm{~d} v \mathrm{~d} x=\int_{\mathbb{R}^{2}} \int_{\mathbb{R}^{2}}\left(f^{\text {in }}\right)^{2} \mathrm{~d} v \mathrm{~d} x, \quad t \in \mathbb{R}_{+} \\
\sup _{0<\varepsilon<1, t \in \mathbb{R}_{+}}\left\{\int_{\mathbb{R}^{2}} \int_{\mathbb{R}^{2}} \frac{|v|^{2}}{2} f^{\varepsilon}(t, x, v) \mathrm{d} v \mathrm{~d} x+\int_{\mathbb{R}^{2}} \frac{\left|\nabla_{x} \phi^{\varepsilon}(t, x)\right|^{2}}{2} \mathrm{~d} x\right\}<+\infty \\
\sup _{\varepsilon>0}\left\|\rho^{\varepsilon}\right\|_{L^{\infty}\left([0, t] \times \mathbb{R}^{2}\right)}<+\infty, \sup _{0<\varepsilon<1}\left\|\nabla_{x} \phi^{\varepsilon}\right\|_{L^{\infty}\left([0, t] ; W^{1, p}\left(\mathbb{R}^{2}\right)\right)}<+\infty, \quad t \in \mathbb{R}_{+}, \quad 1 \leq p<+\infty .
\end{gathered}
$$

Remark 3.1 We consider the function $F: \mathbb{R}_{+} \times \mathbb{R}_{+} \rightarrow \mathbb{R}$ defined for any $(a, r) \in \mathbb{R}_{+} \times \mathbb{R}_{+}$ by

$$
F(a, r)= \begin{cases}F^{\text {in }}(0), & 0 \leq r<a \\ F^{\text {in }}(r-a), & r \geq a .\end{cases}
$$

The function $F$ belongs to $C\left(\mathbb{R}_{+} ; L^{1}\left(\mathbb{R}_{+} ; r \mathrm{~d} r\right)\right)$, is non increasing with respect to $r$, and non decreasing with respect to a. Thanks to the inequality

$$
\left|V^{\varepsilon}(0 ; t, x, v)\right| \geq|v|-\int_{0}^{t}\left\|E^{\varepsilon}(\tau)\right\|_{L^{\infty}\left(\mathbb{R}^{2}\right)} \mathrm{d} \tau
$$

it is easily seen that for any $0<\varepsilon<1$ and $(t, x, v) \in \mathbb{R}_{+} \times \mathbb{R}^{2} \times \mathbb{R}^{2}$ we have

$$
\begin{aligned}
f^{\varepsilon}(t, x, v) & =f^{\text {in }}\left(X^{\varepsilon}(0 ; t, x, v), V^{\varepsilon}(0 ; t, x, v)\right) \leq F^{\text {in }}\left(\left|V^{\varepsilon}(0 ; t, x, v)\right|\right) \\
& \leq F\left(\int_{0}^{t}\left\|E^{\varepsilon}(\tau)\right\|_{L^{\infty}\left(\mathbb{R}^{2}\right)} \mathrm{d} \tau,|v|\right) \leq F\left(t \sup _{0<\varepsilon^{\prime}<1}\left\|E^{\varepsilon^{\prime}}\right\|_{L^{\infty}\left([0, t] \times \mathbb{R}^{2}\right)},|v|\right) .
\end{aligned}
$$

In order to get stability, when $\varepsilon \searrow 0$, we need to filter out the fast time oscillations, due to the magnetic acceleration $\frac{\omega_{c}}{\varepsilon}{ }^{\perp} v$. At any time $t \in \mathbb{R}_{+}$we introduce the densities $\tilde{f}^{\varepsilon}(t)$ in the new coordinates

$$
\widetilde{x}=x+\frac{{ }^{\perp} v}{\omega_{c}}, \widetilde{v}=\mathcal{R}\left(\omega_{c} t / \varepsilon\right) v
$$


that is

$$
f^{\varepsilon}(t, x, v)=\tilde{f}^{\varepsilon}\left(t, x+\frac{{ }^{\perp} v}{\omega_{c}}, \mathcal{R}\left(\omega_{c} t / \varepsilon\right) v\right), \quad(t, x, v) \in \mathbb{R}_{+} \times \mathbb{R}^{2} \times \mathbb{R}^{2}, \quad \varepsilon>0 .
$$

A straightforward application of the chain rule and standard manipulations with smooth test functions allow us to transform the problem (9), (10), (11) into the problem

$$
\begin{gathered}
\partial_{t} \tilde{f}^{\varepsilon}-\frac{{ }^{\perp} \nabla_{x} \phi^{\varepsilon}}{\omega_{c}}(t, x) \cdot \nabla_{\widetilde{x}} \tilde{f}^{\varepsilon}-\mathcal{R}\left(\omega_{c} t / \varepsilon\right) \nabla_{x} \phi^{\varepsilon}(t, x) \cdot \nabla_{\widetilde{v}} \tilde{f}^{\varepsilon}=0, \quad x=\widetilde{x}-\omega_{c}^{-1} \mathcal{R}\left(-\omega_{c} t / \varepsilon\right){ }^{\perp} \widetilde{v} \\
-\Delta_{x} \phi^{\varepsilon}=\int_{\mathbb{R}^{2}} \tilde{f}^{\varepsilon}\left(t, x+\frac{{ }^{\perp} v}{\omega_{c}}, \mathcal{R}\left(\omega_{c} t / \varepsilon\right) v\right) \mathrm{d} v, \quad(t, x) \in \mathbb{R}_{+} \times \mathbb{R}^{2} \\
\tilde{f}^{\varepsilon}(0, \widetilde{x}, \widetilde{v})=f^{\text {in }}\left(\widetilde{x}-\frac{{ }^{\perp} \widetilde{v}}{\omega_{c}}, \widetilde{v}\right), \quad(\widetilde{x}, \widetilde{v}) \in \mathbb{R}^{2} \times \mathbb{R}^{2}
\end{gathered}
$$

Observe that for any $s \in \mathbb{R}$, the transformation $(x, v) \rightarrow\left(\widetilde{x}=x+\frac{\perp_{v}}{\omega_{c}}, \widetilde{v}=\mathcal{R}\left(\omega_{c} s\right) v\right)$ has Jacobian determinant equal to 1 and thus it preserves the Lebesgue measure $\mathrm{d} \widetilde{x} \mathrm{~d} \widetilde{v}=\mathrm{d} x \mathrm{~d} v$. Therefore the $L^{p}$ norms of the new densities $\tilde{f}^{\varepsilon}(t)$ are still conserved in time, for example

$$
\begin{gathered}
\int_{\mathbb{R}^{2}} \int_{\mathbb{R}^{2}} \tilde{f}^{\varepsilon}(t, \widetilde{x}, \widetilde{v}) \mathrm{d} \tilde{v} \mathrm{~d} \tilde{x}=\int_{\mathbb{R}^{2}} \int_{\mathbb{R}^{2}} f^{\varepsilon}(t, x, v) \mathrm{d} v \mathrm{~d} x=\int_{\mathbb{R}^{2}} \int_{\mathbb{R}^{2}} f^{\text {in }}(x, v) \mathrm{d} v \mathrm{~d} x, \quad t \in \mathbb{R}_{+} \\
\int_{\mathbb{R}^{2}} \int_{\mathbb{R}^{2}}\left(\tilde{f}^{\varepsilon}(t, \widetilde{x}, \widetilde{v})\right)^{2} \mathrm{~d} \tilde{v} \mathrm{~d} \tilde{x}=\int_{\mathbb{R}^{2}} \int_{\mathbb{R}^{2}}\left(f^{\varepsilon}(t, x, v)\right)^{2} \mathrm{~d} v \mathrm{~d} x=\int_{\mathbb{R}^{2}} \int_{\mathbb{R}^{2}}\left(f^{\text {in }}(x, v)\right)^{2} \mathrm{~d} v \mathrm{~d} x, \quad t \in \mathbb{R}_{+} .
\end{gathered}
$$

It is easily seen that $\tilde{f}^{\varepsilon}(t), f^{\varepsilon}(t)$ have the same kinetic energy and satisfy the same $L^{\infty}$ bound

$$
\begin{gathered}
\int_{\mathbb{R}^{2}} \int_{\mathbb{R}^{2}} \tilde{f}^{\varepsilon}(t, \widetilde{x}, \widetilde{v}) \frac{|\widetilde{v}|^{2}}{2} \mathrm{~d} \tilde{v} \mathrm{~d} \tilde{x}=\int_{\mathbb{R}^{2}} \int_{\mathbb{R}^{2}} f^{\varepsilon}(t, x, v) \frac{|v|^{2}}{2} \mathrm{~d} v \mathrm{~d} x, \quad t \in \mathbb{R}_{+} \\
\left\|\tilde{f}^{\varepsilon}\right\|_{L^{\infty}\left(\mathbb{R}_{+} \times \mathbb{R}^{2} \times \mathbb{R}^{2}\right)}=\left\|f^{\varepsilon}\right\|_{L^{\infty}\left(\mathbb{R}_{+} \times \mathbb{R}^{2} \times \mathbb{R}^{2}\right)} \leq\left\|F^{\mathrm{in}}\right\|_{L^{\infty}\left(\mathbb{R}_{+}\right)} .
\end{gathered}
$$

Remark 3.2 With the notations of Remark 3.1, we have for any $0<\varepsilon<1$

$$
\begin{aligned}
\tilde{f}^{\varepsilon}(t, \widetilde{x}, \widetilde{v}) & =f^{\varepsilon}\left(t, \widetilde{x}-\omega_{c}^{-1} \mathcal{R}\left(-\omega_{c} t / \varepsilon\right){ }^{\perp} \widetilde{v}, \mathcal{R}\left(-\omega_{c} t / \varepsilon\right) \widetilde{v}\right) \\
& \leq F\left(t \sup _{0<\varepsilon^{\prime}<1}\left\|E^{\varepsilon^{\prime}}\right\|_{L^{\infty}\left([0, t] \times \mathbb{R}^{2}\right)},|\widetilde{v}|\right), \quad(t, \widetilde{x}, \widetilde{v}) \in \mathbb{R}_{+} \times \mathbb{R}^{2} \times \mathbb{R}^{2}
\end{aligned}
$$

and thus any weak $\star$ limit density $f$ in $L^{\infty}$ will satisfy

$$
\tilde{f}(t, \widetilde{x}, \widetilde{v}) \leq F\left(T \sup _{0<\varepsilon^{\prime}<1}\left\|E^{\varepsilon^{\prime}}\right\|_{L^{\infty}\left([0, T] \times \mathbb{R}^{2}\right)},|\widetilde{v}|\right), \quad(t, \widetilde{x}, \widetilde{v}) \in[0, T] \times \mathbb{R}^{2} \times \mathbb{R}^{2} .
$$

Actually the equation (36) may be written in conservative form. Indeed, let us consider the functions

$$
\widetilde{\phi}^{\varepsilon}(t, \widetilde{x}, \widetilde{v})=\phi^{\varepsilon}\left(t, \widetilde{x}-\omega_{c}^{-1} \mathcal{R}\left(-\omega_{c} t / \varepsilon\right)^{\perp} \widetilde{v}\right)
$$

and notice that

$$
\frac{{ }^{\perp} \nabla_{\widetilde{x}} \widetilde{\phi}^{\varepsilon}(t, \widetilde{x}, \widetilde{v})}{\omega_{c}}=\frac{{ }^{\perp} \nabla_{x} \phi^{\varepsilon}(t, x)}{\omega_{c}}, \omega_{c}{ }^{\perp} \nabla_{\widetilde{v}} \widetilde{\phi}^{\varepsilon}=-\mathcal{R}\left(\omega_{c} t / \varepsilon\right) \nabla_{x} \phi^{\varepsilon}(t, x) .
$$


Therefore the equation (36) writes

$$
\partial_{t} \tilde{f}^{\varepsilon}-\frac{{ }^{\perp} \nabla_{\widetilde{x}} \widetilde{\phi}^{\varepsilon}}{\omega_{c}} \cdot \nabla_{\widetilde{x}} \tilde{f}^{\varepsilon}+\omega_{c}{ }^{\perp} \nabla_{\widetilde{v}} \widetilde{\phi}^{\varepsilon} \cdot \nabla_{\widetilde{v}} \tilde{f}^{\varepsilon}=0, \quad(t, \widetilde{x}, \widetilde{v}) \in \mathbb{R}_{+} \times \mathbb{R}^{2} \times \mathbb{R}^{2}
$$

or equivalently

$$
\partial_{t} \tilde{f}^{\varepsilon}-\operatorname{div}_{\widetilde{x}}\left(\tilde{f}^{\varepsilon} \omega_{c}^{-1}{ }^{\perp} \nabla_{\widetilde{x}} \widetilde{\phi}^{\varepsilon}\right)+\operatorname{div}_{\widetilde{v}}\left(\tilde{f}^{\varepsilon} \omega_{c}{ }^{\perp} \nabla_{\widetilde{v}} \widetilde{\phi}^{\varepsilon}\right)=0, \quad(t, \widetilde{x}, \widetilde{v}) \in \mathbb{R}_{+} \times \mathbb{R}^{2} \times \mathbb{R}^{2} .
$$

Remark 3.3 The solutions $\left(\tilde{f}^{\varepsilon}\right)_{0<\varepsilon<1}$ propagate with finite speed on any interval $[0, T]$, uniformly with respect to the parameter $\varepsilon \in] 0,1\left[\right.$. This is a consequence of the $L^{\infty}$ bound (35) for the electric fields. For any $T \in \mathbb{R}_{+}, t \in[0, T], 0<\varepsilon<1$ we prove that

$$
\int_{\mathbb{R}^{2}} \int_{\mathbb{R}^{2}} \tilde{f}^{\varepsilon}(t, \widetilde{x}, \widetilde{v}) \mathbf{1}_{\left\{\sqrt{|\widetilde{x}|^{2}+|\widetilde{v}|^{2}}>R\right\}} \mathrm{d} \tilde{v} \mathrm{~d} \tilde{x} \leq \int_{\mathbb{R}^{2}} \int_{\mathbb{R}^{2}} f^{\text {in }}\left(\widetilde{x}-\frac{\perp \widetilde{v}}{\omega_{c}}, \widetilde{v}\right) \mathbf{1}_{\left\{\sqrt{|\widetilde{x}|^{2}+|\widetilde{v}|^{2}}>R-t E_{T}\right\}} \mathrm{d} \tilde{v} \mathrm{~d} \tilde{x}
$$

and

$\int_{\mathbb{R}^{2}} \int_{\mathbb{R}^{2}}\left(\tilde{f}^{\varepsilon}(t, \widetilde{x}, \widetilde{v})\right)^{2} \mathbf{1}_{\left\{\sqrt{|\widetilde{x}|^{2}+|\widetilde{v}|^{2}}>R\right\}} \mathrm{d} \tilde{v} \mathrm{~d} \tilde{x} \leq \int_{\mathbb{R}^{2}} \int_{\mathbb{R}^{2}}\left(f^{\text {in }}\left(\widetilde{x}-\frac{\perp \widetilde{v}}{\omega_{c}}, \widetilde{v}\right)\right)^{2} \mathbf{1}_{\left\{\sqrt{|\widetilde{x}|^{2}+|\widetilde{v}|^{2}}>R-t E_{T}\right\}} \mathrm{d} \tilde{v} \mathrm{~d} \tilde{x}$ with

$$
E_{T}=\sqrt{1+\omega_{c}^{-2}} \sup _{0<\varepsilon<1}\left\|E^{\varepsilon}\right\|_{L^{\infty}\left([0, T] \times \mathbb{R}^{2}\right)}<+\infty .
$$

As the density $(\widetilde{x}, \widetilde{v}) \rightarrow f^{\text {in }}\left(\widetilde{x}-\frac{\perp \widetilde{v}}{\omega_{c}}, \widetilde{v}\right)$ belongs to $L^{1}\left(\mathbb{R}^{2} \times \mathbb{R}^{2}\right) \cap L^{2}\left(\mathbb{R}^{2} \times \mathbb{R}^{2}\right)$, we deduce that

$$
\lim _{R \rightarrow+\infty} \sup _{0<\varepsilon<1, t \in[0, T]}\left\{\left\|\tilde{f}^{\varepsilon}(t)\right\|_{L^{1}\left(\mathcal{C} B_{R}\right)}+\left\|\tilde{f}^{\varepsilon}(t)\right\|_{L^{2}\left(\complement B_{R}\right)}\right\}=0, \quad T \in \mathbb{R}_{+}
$$

where $\complement B_{R}=\left\{(\widetilde{x}, \widetilde{v}):|\widetilde{x}|^{2}+|\widetilde{v}|^{2} \geq R^{2}\right\}$.

Remark 3.4 Notice that if the initial density $f^{\text {in }}=f^{\text {in }}(x, v)$ is compactly supported in $\mathbb{R}^{2} \times \mathbb{R}^{2}$, then the density $(\widetilde{x}, \widetilde{v}) \rightarrow f^{\mathrm{in}}\left(\widetilde{x}-\perp \widetilde{v} / \omega_{c}, \widetilde{v}\right)$ remains compactly supported in $\mathbb{R}^{2} \times \mathbb{R}^{2}$. By the finite speed propagation property (cf. Remark 3.3), we deduce that for any $T \in \mathbb{R}_{+}$, the densities $\left.\tilde{f}^{\varepsilon}\right|_{[0, T] \times \mathbb{R}^{2} \times \mathbb{R}^{2}}$, and therefore $\left.f^{\varepsilon}\right|_{[0, T] \times \mathbb{R}^{2} \times \mathbb{R}^{2}}$, remain compactly supported, uniformly with respect to $\varepsilon \in] 0,1\left[\right.$. In particular the charge densities $\left.\rho^{\varepsilon}\right|_{[0, T] \times \mathbb{R}^{2}}$ remain compactly supported, uniformly with respect to $\varepsilon \in] 0,1\left[\right.$, and therefore the electric potentials $\phi^{\varepsilon}(t)=e \star \rho^{\varepsilon}(t)$ remain locally bounded on $[0, T] \times \mathbb{R}^{2}$, uniformly with respect to $\left.\varepsilon \in\right] 0,1[$.

For any test function $\psi \in C_{c}^{1}(] 0, T\left[\times \mathbb{R}^{2} \times \mathbb{R}^{2}\right)$, we can write thanks to the uniform estimates of $\left(\tilde{f}^{\varepsilon}\right)_{\varepsilon>0}$ in $L^{\infty}\left(\mathbb{R}_{+} ; L^{2}\left(\mathbb{R}^{2} \times \mathbb{R}^{2}\right)\right)$ and of $\left(E^{\varepsilon}\right)_{\varepsilon>0}$ in $L^{\infty}\left([0, T] \times \mathbb{R}^{2}\right)$

$$
\begin{aligned}
& \int_{0}^{T} \int_{\mathbb{R}^{2}} \int_{\mathbb{R}^{2}} \tilde{f}^{\varepsilon} \partial_{t} \psi \mathrm{d} \tilde{v} \mathrm{~d} \tilde{x} \mathrm{~d} t=\int_{0}^{T} \int_{\mathbb{R}^{2}} \int_{\mathbb{R}^{2}} \tilde{f}^{\varepsilon}\left[\frac{{ }^{\perp} \nabla_{x} \phi^{\varepsilon}}{\omega_{c}}(t, x) \cdot \nabla_{\widetilde{x}} \psi+\mathcal{R}\left(\omega_{c} t / \varepsilon\right) \nabla_{x} \phi^{\varepsilon}(t, x) \cdot \nabla_{\widetilde{v}} \psi\right] \mathrm{d} \tilde{v} \mathrm{~d} \tilde{x} \mathrm{~d} t \\
& \leq \int_{0}^{T}\left\|\tilde{f}^{\varepsilon}(t)\right\|_{L^{2}\left(\mathbb{R}^{2} \times \mathbb{R}^{2}\right)}\left[\frac{\left\|\nabla_{x} \phi^{\varepsilon}(t)\right\|_{L^{\infty}}}{\left|\omega_{c}\right|}\left\|\nabla_{\widetilde{x}} \psi(t)\right\|_{L^{2}}+\left\|\nabla_{x} \phi^{\varepsilon}(t)\right\|_{L^{\infty}}\left\|\nabla_{\widetilde{v}} \psi(t)\right\|_{L^{2}}\right] \mathrm{d} t \\
& \leq C_{T}\|\psi\|_{L^{1}\left([0, T] ; H^{1}\left(\mathbb{R}^{2} \times \mathbb{R}^{2}\right)\right)} .
\end{aligned}
$$


We deduce that $\left\{\partial_{t} \tilde{f}^{\varepsilon}: 0<\varepsilon<1\right\}$ is bounded in $L^{\infty}\left([0, T] ; H^{-1}\left(B_{R}\right)\right)$ for any ball $B_{R}=$ $\left\{(\widetilde{x}, \widetilde{v}):|\widetilde{x}|^{2}+|\widetilde{v}|^{2}<R^{2}\right\}$. As the set $\left\{\tilde{f}^{\varepsilon}, 0<\varepsilon<1\right\}$ is bounded in $L^{\infty}\left([0, T] ; L^{2}\left(B_{R}\right)\right)$, we obtain by standard compactness results $[3,16]$ that $\left\{\tilde{f}^{\varepsilon}, 0<\varepsilon<1\right\}$ is relatively compact in $L^{\infty}\left([0, T] ; H^{-1}\left(B_{R}\right)\right)$ for any ball $B_{R} \subset \mathbb{R}^{2} \times \mathbb{R}^{2}$ and any $T \in \mathbb{R}_{+}$. Based on the above compactness property of the family $\left(\tilde{f}^{\varepsilon}\right)_{0<\varepsilon<1}$ we can pass to the limit, when $\varepsilon \searrow 0$, in the problems $(36),(37),(38)$. Let $\left.\left(\varepsilon_{k}\right)_{k \in \mathbb{N}} \subset\right] 0,1[$ be a sequence converging to 0 and $\tilde{f} \in$ $L^{\infty}\left(\mathbb{R}_{+} ; L^{2}\left(\mathbb{R}^{2} \times \mathbb{R}^{2}\right)\right)$ such that $\left(\tilde{f}^{\varepsilon_{k}}\right)_{k}$ converges to $\tilde{f}$ weakly $\star$ in $L^{\infty}\left(\mathbb{R}_{+} ; L^{2}\left(\mathbb{R}^{2} \times \mathbb{R}^{2}\right)\right)$ and $L^{\infty}\left(\mathbb{R}_{+} \times \mathbb{R}^{2} \times \mathbb{R}^{2}\right)$, and $\left(\tilde{f}^{\varepsilon}\right)_{k}$ converges to $\tilde{f}$ strongly in $L^{\infty}\left([0, T] ; H^{-1}\left(B_{R}\right)\right)$ for any ball $B_{R} \subset \mathbb{R}^{2} \times \mathbb{R}^{2}$ and any $T \in \mathbb{R}_{+}$. By weak $\star$ convergence we obtain immediately that $\tilde{f} \geq 0$ and

$$
\begin{aligned}
& \|\tilde{f}\|_{L^{\infty}} \leq \liminf _{k \rightarrow+\infty}\left\|\tilde{f}^{\varepsilon_{k}}\right\|_{L^{\infty}}=\liminf _{k \rightarrow+\infty}\left\|f^{\varepsilon_{k}}\right\|_{L^{\infty}} \leq\left\|F^{\mathrm{in}}\right\|_{L^{\infty}} \\
& \|\tilde{f}\|_{L^{\infty}\left(\mathbb{R}_{+} ; L^{1}\left(\mathbb{R}^{2} \times \mathbb{R}^{2}\right)\right)} \leq\left\|f^{\mathrm{in}}\right\|_{L^{1}\left(\mathbb{R}^{2} \times \mathbb{R}^{2}\right)}, \quad\|\tilde{f}\|_{L^{\infty}\left(\mathbb{R}_{+} ; L^{2}\left(\mathbb{R}^{2} \times \mathbb{R}^{2}\right)\right)} \leq\left\|f^{\mathrm{in}}\right\|_{L^{2}\left(\mathbb{R}^{2} \times \mathbb{R}^{2}\right)} \\
& \left\|\int_{\mathbb{R}^{2}} \int_{\mathbb{R}^{2}} \tilde{f}(\cdot, \widetilde{x}, \widetilde{v}) \frac{|\widetilde{v}|^{2}}{2} \mathrm{~d} \tilde{v} \mathrm{~d} \tilde{x}\right\|_{L^{\infty}\left(\mathbb{R}_{+}\right)} \leq \liminf _{k \rightarrow+\infty}\left\|\int_{\mathbb{R}^{2}} \int_{\mathbb{R}^{2}} \tilde{f}^{\varepsilon_{k}}(\cdot, \widetilde{x}, \widetilde{v}) \frac{|\widetilde{v}|^{2}}{2} \mathrm{~d} \tilde{v} \mathrm{~d} \tilde{x}\right\|_{L^{\infty}\left(\mathbb{R}_{+}\right)} \\
& =\liminf _{k \rightarrow+\infty}\left\|\int_{\mathbb{R}^{2}} \int_{\mathbb{R}^{2}} f^{\varepsilon_{k}}(\cdot, x, v) \frac{|v|^{2}}{2} \mathrm{~d} v \mathrm{~d} x\right\|_{L^{\infty}\left(\mathbb{R}_{+}\right)}<+\infty .
\end{aligned}
$$

Moreover, thanks to the finite speed propagation property, we check that

$$
\int_{\mathbb{R}^{2}} \int_{\mathbb{R}^{2}} \tilde{f}(t, \widetilde{x}, \widetilde{v}) \mathrm{d} \tilde{v} \mathrm{~d} \tilde{x}=\int_{\mathbb{R}^{2}} \int_{\mathbb{R}^{2}} f^{\text {in }}(x, v) \mathrm{d} v \mathrm{~d} x, \quad t \in \mathbb{R}_{+} .
$$

The weak formulation of (36), (37) written for smooth test functions $\psi \in C_{c}^{2}\left(\mathbb{R}_{+} \times \mathbb{R}^{2} \times \mathbb{R}^{2}\right)$ yields

$$
\begin{gathered}
-\int_{\mathbb{R}_{+}} \int_{\mathbb{R}^{2}} \int_{\mathbb{R}^{2}} \tilde{f}^{\varepsilon_{k}} \partial_{t} \psi \mathrm{d} \tilde{v} \mathrm{~d} \tilde{x} \mathrm{~d} t-\int_{\mathbb{R}^{2}} \int_{\mathbb{R}^{2}} \tilde{f}(0, \widetilde{x}, \widetilde{v}) \psi(0, \widetilde{x}, \widetilde{v}) \mathrm{d} \tilde{v} \mathrm{~d} \tilde{x}+\int_{\mathbb{R}_{+}} \int_{\mathbb{R}^{2}} \int_{\mathbb{R}^{2}} \tilde{f}^{\varepsilon_{k}}(t, \tilde{x}, \widetilde{v}) \\
\times\left[\frac{{ }^{\perp} \nabla_{x} \phi^{\varepsilon_{k}}}{\omega_{c}}(t, x) \cdot \nabla_{\widetilde{x}} \psi+\mathcal{R}\left(\omega_{c} t / \varepsilon_{k}\right) \nabla_{x} \phi^{\varepsilon_{k}}(t, x) \cdot \nabla_{\widetilde{v}} \psi\right] \mathrm{d} \tilde{v} \mathrm{~d} \tilde{x} \mathrm{~d} t=0
\end{gathered}
$$

Clearly, the weak $\star$ convergence of $\left(\tilde{f}^{\varepsilon_{k}}\right)_{k}$ in $L^{\infty}\left(\mathbb{R}_{+} ; L^{2}\left(\mathbb{R}^{2} \times \mathbb{R}^{2}\right)\right)$ implies

$$
\lim _{k \rightarrow+\infty} \int_{\mathbb{R}_{+}} \int_{\mathbb{R}^{2}} \int_{\mathbb{R}^{2}} \tilde{f}^{\varepsilon_{k}} \partial_{t} \psi \mathrm{d} \tilde{v} \mathrm{~d} \tilde{x} \mathrm{~d} t=\int_{\mathbb{R}_{+}} \int_{\mathbb{R}^{2}} \int_{\mathbb{R}^{2}} \tilde{f} \partial_{t} \psi \mathrm{d} \tilde{v} \mathrm{~d} \tilde{x} \mathrm{~d} t
$$

We are done if we handle the bilinear terms entering the last integral in (39). We claim that $\left({ }^{\perp} \nabla_{x} \phi^{\varepsilon_{k}}(t, x) \cdot \nabla_{\widetilde{x}} \psi\right)_{k}$ and $\left(\mathcal{R}\left(\omega_{c} t / \varepsilon_{k}\right) \nabla_{x} \phi^{\varepsilon_{k}}(t, x) \cdot \nabla_{\widetilde{v}} \psi\right)_{k}$ are bounded in $L^{1}\left(\mathbb{R}_{+} ; H^{1}\left(\mathbb{R}^{2} \times\right.\right.$ $\left.\mathbb{R}^{2}\right)$ ). Indeed, taking $T, R>0$ such that supp $\psi \subset\left[0, T\left[\times B_{R}\right.\right.$, we obtain, thanks to the 
uniform estimates in Proposition 3.1

$$
\begin{aligned}
\sup _{k} & \left\|^{\perp} \nabla_{x} \phi^{\varepsilon_{k}} \cdot \nabla_{\widetilde{x}} \psi\right\|_{L^{1}\left(\mathbb{R}_{+} ; L^{2}\left(\mathbb{R}^{2} \times \mathbb{R}^{2}\right)\right)} \\
& =\sup _{k} \int_{\mathbb{R}_{+}}\left(\int_{\mathbb{R}^{2}} \int_{\mathbb{R}^{2}}\left({ }^{\perp} \nabla_{x} \phi^{\varepsilon_{k}}\left(t, \widetilde{x}-\omega_{c}^{-1} \mathcal{R}\left(-\omega_{c} t / \varepsilon_{k}\right){ }^{\perp} \widetilde{v}\right) \cdot \nabla_{\widetilde{x}} \psi\right)^{2} \mathrm{~d} \tilde{v} \mathrm{~d} \tilde{x}\right)^{1 / 2} \mathrm{~d} t \\
& \leq \sup _{k} \int_{0}^{T}\left\|\nabla_{\widetilde{x}} \psi\right\|_{L^{\infty}}\left(\int_{B_{R}}\left|\nabla_{x} \phi^{\varepsilon_{k}}\left(t, \widetilde{x}-\omega_{c}^{-1} \mathcal{R}\left(-\omega_{c} t / \varepsilon_{k}\right){ }^{\perp} \widetilde{v}\right)\right|^{2} \mathrm{~d} \widetilde{v} \mathrm{~d} \widetilde{x}\right)^{1 / 2} \mathrm{~d} t \\
& \leq\left\|\nabla_{\widetilde{x}} \psi\right\|_{L^{\infty}} \sup _{k} \int_{0}^{T}\left(\int_{|\widetilde{v}| \leq R} \int_{|\widetilde{x}| \leq R}\left|\nabla_{x} \phi^{\varepsilon_{k}}\left(t, \widetilde{x}-\omega_{c}^{-1} \mathcal{R}\left(-\omega_{c} t / \varepsilon_{k}\right){ }^{\perp} \widetilde{v}\right)\right|^{2} \mathrm{~d} \widetilde{x} \mathrm{~d} \widetilde{v}\right)^{1 / 2} \mathrm{~d} t \\
& \leq T\left\|\nabla_{\widetilde{x}} \psi\right\|_{L^{\infty}} \sqrt{\pi R^{2}} \sup _{t \in \mathbb{R}_{+}, k}\left\|\nabla_{x} \phi^{\varepsilon_{k}}(t)\right\|_{L^{2}\left(\mathbb{R}^{2}\right)}<+\infty .
\end{aligned}
$$

Similarly one gets

$$
\sup _{k}\left\|\mathcal{R}\left(\omega_{c} t / \varepsilon_{k}\right) \nabla_{x} \phi^{\varepsilon_{k}} \cdot \nabla_{\widetilde{v}} \psi\right\|_{L^{1}\left(\mathbb{R}_{+} ; L^{2}\left(\mathbb{R}^{2} \times \mathbb{R}^{2}\right)\right)} \leq T\left\|\nabla_{\widetilde{v}} \psi\right\|_{L^{\infty}} \sqrt{\pi R^{2}} \sup _{t \in \mathbb{R}_{+}, k}\left\|\nabla_{x} \phi^{\varepsilon_{k}}(t)\right\|_{L^{2}\left(\mathbb{R}^{2}\right)}
$$

saying that the sequence $\left(\xi_{k}\right)_{k}$ given by

$\xi_{k}(t, \widetilde{x}, \widetilde{v})=\frac{{ }^{\perp} \nabla_{x} \phi^{\varepsilon_{k}}}{\omega_{c}} \cdot \nabla_{\widetilde{x}} \psi+\mathcal{R}\left(\omega_{c} t / \varepsilon_{k}\right) \nabla_{x} \phi^{\varepsilon_{k}} \cdot \nabla_{\widetilde{v}} \psi=\left(\frac{{ }^{\perp} \nabla_{\widetilde{x}} \widetilde{\phi}^{\varepsilon_{k}}}{\omega_{c}},-\omega_{c}{ }^{\perp} \nabla_{\widetilde{v}} \widetilde{\phi}^{\varepsilon_{k}}\right) \cdot \nabla_{\widetilde{x}, \widetilde{v}} \psi, \quad k \in \mathbb{N}$ remains in a bounded set of $L^{1}\left(\mathbb{R}_{+} ; L^{2}\left(\mathbb{R}^{2} \times \mathbb{R}^{2}\right)\right)$. We claim that $\left(\nabla_{\widetilde{x}, \widetilde{v}} \xi_{k}\right)_{k}$ is also bounded in $L^{1}\left(\mathbb{R}_{+} ; L^{2}\left(\mathbb{R}^{2} \times \mathbb{R}^{2}\right)\right)$. For that it is enough to check that all second derivatives of $\phi^{\varepsilon_{k}}$ are uniformly bounded in $L^{\infty}\left([0, T] ; L^{2}\left(B_{R}\right)\right)$

$$
\sup _{t \in \mathbb{R}_{+}, k}\left\|\partial_{\widetilde{x}, \widetilde{v}} \nabla_{\widetilde{x}, \widetilde{v}} \widetilde{\phi}^{\varepsilon k}(t)\right\|_{L^{2}\left(B_{R}\right)}<+\infty .
$$

It is easily seen that

$$
\begin{gathered}
\partial_{\widetilde{x}} \nabla_{\widetilde{x}} \widetilde{\phi}^{\varepsilon_{k}}=\partial_{x} \nabla_{x} \phi^{\varepsilon_{k}}, \quad \partial_{\widetilde{v}} \nabla_{\widetilde{v}} \widetilde{\phi}^{\varepsilon_{k}}=\omega_{c}^{-2} \mathcal{R}\left(\omega_{c} t / \varepsilon_{k}+\pi / 2\right) \partial_{x} \nabla_{x} \phi^{\varepsilon_{k}} \mathcal{R}\left(-\omega_{c} t / \varepsilon_{k}-\pi / 2\right) \\
\partial_{\widetilde{v}} \nabla_{\widetilde{x}} \widetilde{\phi}^{\varepsilon_{k}}=-\omega_{c}^{-1} \partial_{x} \nabla_{x} \phi^{\varepsilon_{k}} \mathcal{R}\left(-\omega_{c} t / \varepsilon_{k}-\pi / 2\right), \quad \partial_{\widetilde{x}} \nabla_{\widetilde{v}} \widetilde{\phi}^{\varepsilon_{k}}=-\omega_{c}^{-1} \mathcal{R}\left(\omega_{c} t / \varepsilon_{k}+\pi / 2\right) \partial_{x} \nabla_{x} \phi^{\varepsilon_{k}}
\end{gathered}
$$

and therefore, by Proposition 3.1, we obtain as before

$$
\sup _{t \in \mathbb{R}_{+}, k}\left\|\partial_{x} \nabla_{x} \phi^{\varepsilon_{k}}\left(t, \widetilde{x}-\omega_{c}^{-1} \mathcal{R}\left(-\omega_{c} t / \varepsilon_{k}\right){ }^{\perp} \widetilde{v}\right)\right\|_{L^{2}\left(B_{R}\right)} \leq \sup _{t \in \mathbb{R}_{+}, k} \sqrt{\pi R^{2}}\left\|\partial_{x} \nabla_{x} \phi^{\varepsilon_{k}}(t)\right\|_{L^{2}\left(\mathbb{R}^{2}\right)}
$$

We split the last integral of (39) in two terms

$$
\int_{\mathbb{R}_{+}} \int_{\mathbb{R}^{2}} \int_{\mathbb{R}^{2}} \tilde{f}^{\varepsilon_{k}} \xi_{k} \mathrm{~d} \tilde{v} \mathrm{~d} \tilde{x} \mathrm{~d} t=\int_{\mathbb{R}_{+}} \int_{\mathbb{R}^{2}} \int_{\mathbb{R}^{2}}\left(\tilde{f}^{\varepsilon_{k}}-\tilde{f}\right) \xi_{k} \mathrm{~d} \tilde{v} \mathrm{~d} \tilde{x} \mathrm{~d} t+\int_{\mathbb{R}_{+}} \int_{\mathbb{R}^{2}} \int_{\mathbb{R}^{2}} \tilde{f} \xi_{k} \mathrm{~d} \tilde{v} \mathrm{~d} \tilde{x} \mathrm{~d} t=T_{k}^{1}+T_{k}^{2} .
$$

The compactness of $\left(\tilde{f}^{\varepsilon_{k}}\right)_{k}$ in $L^{\infty}\left([0, T] ; H^{-1}\left(B_{R}\right)\right)$ and the uniform bound of the sequence $\left(\xi_{k}\right)_{k}$ in $L^{1}\left(\mathbb{R}_{+} ; H^{1}\left(\mathbb{R}^{2} \times \mathbb{R}^{2}\right)\right)$ allow us to get rid of $T_{k}^{1}$, as $k \rightarrow+\infty$

$$
\begin{aligned}
\left|T_{k}^{1}\right| & =\left|\int_{0}^{T}\left\langle\tilde{f}^{\varepsilon_{k}}(t)-\tilde{f}(t), \xi_{k}(t)\right\rangle_{H^{-1}\left(B_{R}\right), H_{0}^{1}\left(B_{R}\right)}\right| \mathrm{d} t \\
& \leq\left\|\tilde{f}^{\varepsilon_{k}}-\tilde{f}\right\|_{L^{\infty}\left([0, T] ; H^{-1}\left(B_{R}\right)\right)}\left\|\xi_{k}\right\|_{L^{1}\left([0, T] ; H_{0}^{1}\left(B_{R}\right)\right)} \rightarrow 0, \text { as } k \rightarrow+\infty .
\end{aligned}
$$


We investigate now the convergence of the sequence $\left(T_{k}^{2}\right)_{k}$. Using the fundamental solution (17) of the Laplace operator, we represent the sequence $\left(\xi_{k}\right)_{k}$ as

$$
\begin{aligned}
\xi_{k}(t, \widetilde{x}, \widetilde{v}) & =\nabla_{\widetilde{x}} \int_{\mathbb{R}^{2}} \int_{\mathbb{R}^{2}} e\left(\widetilde{x}-\omega_{c}^{-1} \mathcal{R}\left(-\omega_{c} t / \varepsilon_{k}\right){ }^{\perp} \widetilde{v}-y\right) f^{\varepsilon_{k}}(t, y, w) \mathrm{d} w \mathrm{~d} y \\
& \cdot\left(-\omega_{c}^{-1}{ }^{\perp} \nabla_{\widetilde{x}} \psi+\mathcal{R}\left(-\omega_{c} t / \varepsilon_{k}\right) \nabla_{\widetilde{v}} \psi\right) \\
& =\int_{\mathbb{R}^{2}} \int_{\mathbb{R}^{2}} \nabla e\left(\widetilde{x}-\widetilde{y}-\omega_{c}^{-1} \mathcal{R}\left(-\omega_{c} t / \varepsilon_{k}\right){ }^{\perp}(\widetilde{v}-\widetilde{w})\right) \tilde{f}^{\varepsilon_{k}}(t, \widetilde{y}, \widetilde{w}) \mathrm{d} \tilde{w} \mathrm{~d} \tilde{y} \\
& \cdot\left(-\omega_{c}^{-1}{ }^{\perp} \nabla_{\widetilde{x}} \psi+\mathcal{R}\left(-\omega_{c} t / \varepsilon_{k}\right) \nabla_{\widetilde{v}} \psi\right) .
\end{aligned}
$$

Plugging the above formula for $\xi_{k}$ in the expression of $T_{k}^{2}$, yields

$$
T_{k}^{2}=\int_{\mathbb{R}_{+}} \int_{\mathbb{R}^{2}} \int_{\mathbb{R}^{2}} \tilde{f}^{\varepsilon_{k}}(t, \widetilde{y}, \widetilde{w}) \chi_{k}(t, \widetilde{y}, \widetilde{w}) \mathrm{d} \tilde{w} \mathrm{~d} \tilde{y} \mathrm{~d} t
$$

with

$$
\begin{aligned}
\chi_{k}(t, \widetilde{y}, \widetilde{w}) & =\int_{\mathbb{R}^{2}} \int_{\mathbb{R}^{2}} \nabla e\left(\widetilde{x}-\widetilde{y}-\omega_{c}^{-1} \mathcal{R}\left(-\omega_{c} t / \varepsilon_{k}\right){ }^{\perp}(\widetilde{v}-\widetilde{w})\right) \cdot\left(-\omega_{c}^{-1}{ }^{\perp} \nabla_{\widetilde{x}} \psi+\mathcal{R}\left(-\omega_{c} t / \varepsilon_{k}\right) \nabla_{\widetilde{v}} \psi\right) \\
& \times \tilde{f}(t, \widetilde{x}, \widetilde{v}) \mathrm{d} \tilde{v} \mathrm{~d} \tilde{x} .
\end{aligned}
$$

Having in mind the compactness of $\left(\tilde{f}^{\varepsilon_{k}}\right)_{k}$ in $L^{\infty}\left([0, T] ; H^{-1}\left(B_{R}\right)\right)$ we write

$$
T_{k}^{2}=\int_{\mathbb{R}_{+}} \int_{\mathbb{R}^{2}} \int_{\mathbb{R}^{2}}\left(\tilde{f}^{\varepsilon_{k}}-\tilde{f}\right) \chi_{k} \mathrm{~d} \tilde{w} \mathrm{~d} \tilde{y} \mathrm{~d} t+\int_{\mathbb{R}_{+}} \int_{\mathbb{R}^{2}} \int_{\mathbb{R}^{2}} \tilde{f} \chi_{k} \mathrm{~d} \tilde{w} \mathrm{~d} \tilde{y} \mathrm{~d} t=T_{k}^{3}+T_{k}^{4}
$$

In order to pass to the limit, when $k \rightarrow+\infty$, in $T_{k}^{3}$, we need to estimate $\left(\chi_{k}\right)_{k}$ uniformly in $L^{1}\left([0, T] ; H_{0}^{1}\left(B_{R}\right)\right)$. Taking into account that $\nabla e(-z)=-\nabla e(z)$, we obtain

$$
\begin{aligned}
\chi_{k}(t, \widetilde{y}, \widetilde{w}) & =\int_{\mathbb{R}^{2}} \int_{\mathbb{R}^{2}} \nabla e\left(\widetilde{y}-\widetilde{x}-\omega_{c}^{-1} \mathcal{R}\left(-\omega_{c} t / \varepsilon_{k}\right){ }^{\perp}(\widetilde{w}-\widetilde{v})\right) \cdot\left(\omega_{c}^{-1}{ }^{\perp} \nabla_{\widetilde{x}} \psi-\mathcal{R}\left(-\omega_{c} t / \varepsilon_{k}\right) \nabla_{\widetilde{v}} \psi\right) \\
& \times \tilde{f}(t, \widetilde{x}, \widetilde{v}) \mathrm{d} \tilde{v} \mathrm{~d} \tilde{x} \\
& =\left(\operatorname{div}_{y} \pi_{k}\right)\left(t, \widetilde{y}-\omega_{c}^{-1} \mathcal{R}\left(-\omega_{c} t / \varepsilon_{k}\right){ }^{\perp} \widetilde{w}\right)
\end{aligned}
$$

where

$$
\begin{gathered}
\pi_{k}(t, y)=\int_{\mathbb{R}^{2}} e(y-x) r_{k}(t, x) \mathrm{d} x, \quad r_{k}(t, x)=\int_{\mathbb{R}^{2}} g_{k}(t, x, v) \mathrm{d} v \\
g_{k}(t, x, v)=\tilde{f}\left(t, x+\frac{{ }^{\perp} v}{\omega_{c}}, \mathcal{R}\left(\omega_{c} t / \varepsilon_{k}\right) v\right) \\
\times\left(\omega_{c}^{-1}{ }^{\perp} \nabla_{\widetilde{x}} \psi(t)-\mathcal{R}\left(-\omega_{c} t / \varepsilon_{k}\right) \nabla_{\widetilde{v}} \psi(t)\right)\left(x+\frac{{ }^{\perp} v}{\omega_{c}}, \mathcal{R}\left(\omega_{c} t / \varepsilon_{k}\right) v\right) .
\end{gathered}
$$

Clearly, the functions

$$
\alpha_{k}(t, x, v)=\left(\omega_{c}^{-1}{ }^{\perp} \nabla_{\widetilde{x}} \psi(t)-\mathcal{R}\left(-\omega_{c} t / \varepsilon_{k}\right) \nabla_{\widetilde{v}} \psi(t)\right)\left(x+\frac{{ }^{\perp} v}{\omega_{c}}, \mathcal{R}\left(\omega_{c} t / \varepsilon_{k}\right) v\right)
$$


are uniformly bounded and supported in $t \in\left[0, T\left[,|v| \leq R,|x| \leq\left(1+\left|\omega_{c}\right|^{-1}\right) R\right.\right.$. The densities $F^{k}(t, x, v):=\tilde{f}\left(t, x+\frac{\perp_{v}}{\omega_{c}}, \mathcal{R}\left(\omega_{c} t / \varepsilon_{k}\right) v\right)$ satisfy

$$
\left\|F^{k}\right\|_{L^{\infty}}=\|\tilde{f}\|_{L^{\infty}}, \quad \int_{\mathbb{R}^{2}} \int_{\mathbb{R}^{2}} F^{k}(t, x, v) \mathrm{d} v \mathrm{~d} x=\int_{\mathbb{R}^{2}} \int_{\mathbb{R}^{2}} \tilde{f}(t, \tilde{x}, \widetilde{v}) \mathrm{d} \tilde{v} \mathrm{~d} \tilde{x}=\int_{\mathbb{R}^{2}} \int_{\mathbb{R}^{2}} f^{\text {in }}(x, v) \mathrm{d} v \mathrm{~d} x .
$$

The sequence $\left(r_{k}\right)_{k}$ remains in a bounded set of $L^{\infty}\left(\mathbb{R}_{+} \times \mathbb{R}^{2}\right)$ and $L^{\infty}\left(\mathbb{R}_{+} ; L^{1}\left(\mathbb{R}^{2}\right)\right)$, and therefore in a bounded set of $L^{\infty}\left(\mathbb{R}_{+} ; L^{p}\left(\mathbb{R}^{2}\right)\right)$ for any $1<p<+\infty$. Thanks to (40), we deduce that

$$
\sup _{k \in \mathbb{N}, t \in \mathbb{R}_{+}}\left\|\operatorname{div}_{y} \pi_{k}(t)\right\|_{W^{1, p}\left(\mathbb{R}^{2}\right)}<+\infty, \quad 1<p<+\infty .
$$

In particular the sequence $\left(\operatorname{div}_{y} \pi_{k}\right)_{k}$ is bounded in $L^{\infty}\left(\mathbb{R}_{+} \times \mathbb{R}^{2}\right)$ and in $L^{\infty}\left(\mathbb{R}_{+} ; H^{1}\left(\mathbb{R}^{2}\right)\right)$. We claim that $\lim _{k \rightarrow+\infty} T_{k}^{3}=0$. Appealing to the finite speed propagation property for $\left(\tilde{f}^{\varepsilon_{k}}\right)_{k}$ and to the uniform $L^{\infty}$ bound for $\left(\chi_{k}\right)_{k}$, it is enough to prove that

$$
\lim _{k \rightarrow+\infty} \int_{\mathbb{R}_{+}} \int_{\mathbb{R}^{2}} \int_{\mathbb{R}^{2}}\left(\tilde{f}^{\varepsilon_{k}}-\tilde{f}\right)(t, \widetilde{y}, \widetilde{w}) \theta(\widetilde{y}, \widetilde{w}) \chi_{k}(t, \widetilde{y}, \widetilde{w}) \mathrm{d} \tilde{w} \mathrm{~d} \tilde{y} \mathrm{~d} t=0
$$

for any $\theta \in C_{c}^{1}\left(\mathbb{R}^{2} \times \mathbb{R}^{2}\right)$. Let us consider a $C^{1}$ function $\theta$, such that $\operatorname{supp} \theta \subset B_{S}, 0 \leq \theta \leq 1$. We are done if we check that the sequence $\left(\theta \chi_{k}\right)_{k}$ is bounded in $L^{1}\left([0, T] ; H_{0}^{1}\left(B_{S}\right)\right)$. This comes immediately by the estimate of $\left(\operatorname{div}_{y} \pi_{k}\right)_{k}$ in $L^{\infty}\left(\mathbb{R}_{+} ; H^{1}\left(\mathbb{R}^{2}\right)\right)$

$\sup _{k \in \mathbb{N}, t \in \mathbb{R}_{+}}\left\|\theta \chi_{k}(t)\right\|_{L^{2}\left(\mathbb{R}^{2} \times \mathbb{R}^{2}\right)} \leq \sup _{k \in \mathbb{N}, t \in \mathbb{R}_{+}}\left\|\chi_{k}(t)\right\|_{L^{2}\left(B_{S}\right)} \leq \sup _{k \in \mathbb{N}, t \in \mathbb{R}_{+}} \sqrt{\pi S^{2}}\left\|\operatorname{div}_{y} \pi_{k}(t)\right\|_{L^{2}\left(\mathbb{R}^{2}\right)}<+\infty$ and similarly

$$
\begin{gathered}
\sup _{k \in \mathbb{N}, t \in \mathbb{R}_{+}}\left\|\nabla_{\widetilde{y}, \widetilde{w}}\left(\theta \chi_{k}(t)\right)\right\|_{L^{2}\left(\mathbb{R}^{2} \times \mathbb{R}^{2}\right)} \leq \sup _{k \in \mathbb{N}, t \in \mathbb{R}_{+}}\left\{\left\|\nabla_{\widetilde{y}, \widetilde{w}} \theta\right\|_{L^{\infty}}\left\|\chi_{k}(t)\right\|_{L^{2}\left(B_{S}\right)}+\|\theta\|_{L^{\infty}}\left\|\nabla_{\widetilde{y}, \widetilde{w} \chi_{k}}(t)\right\|_{L^{2}\left(B_{S}\right)}\right\} \\
\leq C(\theta) \sqrt{\pi S^{2}} \sup _{k \in \mathbb{N}, t \in \mathbb{R}_{+}}\left\{\left\|\operatorname{div}_{y} \pi_{k}(t)\right\|_{L^{2}\left(\mathbb{R}^{2}\right)}+\left\|\nabla_{y} \operatorname{div}_{y} \pi_{k}(t)\right\|_{L^{2}\left(\mathbb{R}^{2}\right)}\right\}<+\infty
\end{gathered}
$$

It remains to determine the limit of the sequence $\left(T_{k}^{4}\right)_{k}$, given by

$$
T_{k}^{4}=\int_{\mathbb{R}_{+}} \int_{\mathbb{R}^{2}} \int_{\mathbb{R}^{2}} \tilde{f} \chi_{k} \mathrm{~d} \tilde{w} \mathrm{~d} \tilde{y} \mathrm{~d} t=\int_{\mathbb{R}_{+}} \int_{\mathbb{R}^{2}} \int_{\mathbb{R}^{2}} \int_{\mathbb{R}^{2}} \int_{\mathbb{R}^{2}} \Psi\left(\tilde{x}, \tilde{v}, \tilde{y}, \tilde{w}, t, t / \varepsilon_{k}\right) \mathrm{d} \tilde{v} \mathrm{~d} \tilde{x} \mathrm{~d} \tilde{w} \mathrm{~d} \tilde{y} \mathrm{~d} t
$$

where

$$
\begin{aligned}
\Psi(\widetilde{x}, \widetilde{v}, \widetilde{y}, \widetilde{w}, t, s) & =\tilde{f}(t, \widetilde{x}, \widetilde{v}) \tilde{f}(t, \widetilde{y}, \widetilde{w}) \nabla e\left(\widetilde{x}-\widetilde{y}-\omega_{c}^{-1} \mathcal{R}\left(-\omega_{c} s\right)^{\perp}(\widetilde{v}-\widetilde{w})\right) \\
& \times\left(-\omega_{c}^{-1}{ }^{\perp} \nabla_{\widetilde{x}} \psi+\mathcal{R}\left(-\omega_{c} s\right) \nabla_{\widetilde{v}} \psi\right)
\end{aligned}
$$

Clearly, the function $\Psi$ varies at two time scales. It depends on a slow time variable $t \in \mathbb{R}$ and also on a fast time variable $s=t / \varepsilon \in \mathbb{R}_{s}$. In order to handle the convergence of the sequence $\left(T_{k}^{4}\right)_{k}$, we appeal to a standard result in homogenization theory. 
Proposition 3.2 Let $U=U(z, t, s): \mathcal{O} \times \mathbb{R}_{+} \times \mathbb{R}_{s} \rightarrow \mathbb{R}$ be a function in $L^{1}\left(\mathcal{O} \times \mathbb{R}_{+} ; C_{\#}\left(\mathbb{R}_{s}\right)\right)$, where $\mathcal{O}$ is an open set of $\mathbb{R}^{N}$ and $C_{\#}\left(\mathbb{R}_{s}\right)$ stands for the set of continuous periodic functions of (fixed) period $L>0$. Then we have the convergence

$$
\lim _{\varepsilon \searrow 0} \int_{\mathcal{O}} \int_{\mathbb{R}_{+}}|U(z, t, t / \varepsilon)| \mathrm{d} t \mathrm{~d} z=\frac{1}{L} \int_{\mathcal{O}} \int_{\mathbb{R}_{+}} \int_{0}^{L}|U(z, t, s)| \mathrm{d} s \mathrm{~d} t \mathrm{~d} z .
$$

Proof. We know that the function $(z, t) \rightarrow\|U(z, t, \cdot)\|_{C_{\#}\left(\mathbb{R}_{s}\right)}$ belongs to $L^{1}\left(\mathcal{O} \times \mathbb{R}_{+}\right)$and thus, for almost all $z \in \mathcal{O}$ the function $t \rightarrow\|U(z, t, \cdot)\|_{C_{\#}\left(\mathbb{R}_{s}\right)}$ belongs to $L^{1}\left(\mathbb{R}_{+}\right)$. Therefore, for almost all $z \in \mathcal{O}$ we have $U(z, \cdot, \cdot) \in L^{1}\left(\mathbb{R}_{+} ; C_{\#}\left(\mathbb{R}_{s}\right)\right)$, and by a classical result (see $\left.[1,15]\right)$ we have

$$
\lim _{\varepsilon \searrow 0} \int_{\mathbb{R}_{+}}|U(z, t, t / \varepsilon)| \mathrm{d} t=\frac{1}{L} \int_{\mathbb{R}_{+}} \int_{0}^{L}|U(z, t, s)| \mathrm{d} s \mathrm{~d} t, \text { for a.a. } z \in \mathcal{O} .
$$

Observe that the family $\left(\int_{\mathbb{R}_{+}}|U(\cdot, t, t / \varepsilon)| \mathrm{d} t\right)_{\varepsilon}$ is dominated by a $L^{1}(\mathcal{O})$ function, that is

$$
\int_{\mathbb{R}_{+}}|U(z, t, t / \varepsilon)| \mathrm{d} t \leq \int_{\mathbb{R}_{+}}\|U(z, t, \cdot)\|_{C_{\#}\left(\mathbb{R}_{s}\right)} \mathrm{d} t, \text { for a.a. } z \in \mathcal{O} .
$$

Therefore, by the dominated convergence theorem, we deduce that

$$
\lim _{\varepsilon \searrow 0} \int_{\mathcal{O}} \int_{\mathbb{R}_{+}}|U(z, t, t / \varepsilon)| \mathrm{d} t \mathrm{~d} z=\frac{1}{L} \int_{\mathcal{O}} \int_{\mathbb{R}_{+}} \int_{0}^{L}|U(z, t, s)| \mathrm{d} s \mathrm{~d} t \mathrm{~d} z .
$$

We intend to apply Proposition 3.2 with the function $\Psi=\Psi(z, t, s)$ where $z=(\widetilde{x}, \widetilde{v}, \widetilde{y}, \widetilde{w}) \in$ $\mathcal{O}=\mathbb{R}^{8}$. Notice that $\Psi$ is $T_{c}$-periodic with respect to the fast time variable $s$, where $T_{c}=$ $2 \pi / \omega_{c}$ is the rescaled cyclotronic period. Since the fundamental solution of the Laplace operator has a singularity at $z=0$, we proceed by approximation. Let us consider

$$
e_{\delta}(z)=-\frac{1}{2 \pi} \ln \sqrt{|z|^{2}+\delta^{2}}, \quad z \in \mathbb{R}^{2} \backslash\{0\}, \quad \delta>0
$$

and

$$
\begin{aligned}
& \Psi_{\delta}(\widetilde{x}, \widetilde{v}, \widetilde{y}, \widetilde{w}, t, s)=\tilde{f}(t, \widetilde{x}, \widetilde{v}) \tilde{f}(t, \widetilde{y}, \widetilde{w}) \nabla e_{\delta}\left(\widetilde{x}-\widetilde{y}-\omega_{c}^{-1} \mathcal{R}\left(-\omega_{c} s\right)^{\perp}(\widetilde{v}-\widetilde{w})\right) \\
& \cdot\left(-\omega_{c}^{-1}{ }^{\perp} \nabla_{\widetilde{x}} \psi+\mathcal{R}\left(-\omega_{c} s\right) \nabla_{\widetilde{v}} \psi\right)
\end{aligned}
$$

Proposition 3.3 For any function $\psi \in C_{c}^{1}\left(\mathbb{R}_{+} \times \mathbb{R}^{2} \times \mathbb{R}^{2}\right)$ and any $\delta>0$ we have

$$
\begin{aligned}
& \lim _{k \rightarrow+\infty} \int_{\mathbb{R}_{+}} \int_{\mathbb{R}^{2}} \int_{\mathbb{R}^{2}} \int_{\mathbb{R}^{2}} \int_{\mathbb{R}^{2}} \Psi_{\delta}\left(\widetilde{x}, \widetilde{v}, \widetilde{y}, \widetilde{w}, t, t / \varepsilon_{k}\right) \mathrm{d} \tilde{v} \mathrm{~d} \tilde{x} \mathrm{~d} \tilde{w} \mathrm{~d} \tilde{y} \mathrm{~d} t \\
& \quad=\frac{1}{T_{c}} \int_{\mathbb{R}_{+}} \int_{\mathbb{R}^{2}} \int_{\mathbb{R}^{2}} \int_{\mathbb{R}^{2}} \int_{\mathbb{R}^{2}} \int_{0}^{T_{c}} \Psi_{\delta}(\widetilde{x}, \widetilde{v}, \widetilde{y}, \widetilde{w}, t, s) \mathrm{d} s \mathrm{~d} \tilde{v} \mathrm{~d} \tilde{x} \mathrm{~d} \tilde{w} \mathrm{~d} \tilde{y} \mathrm{~d} t .
\end{aligned}
$$


Proof. Apply Proposition 3.2 with the function $\Psi_{\delta}(z, t, s)$, where $z=(\widetilde{x}, \widetilde{v}, \widetilde{y}, \widetilde{w}) \in \mathcal{O}=\mathbb{R}^{8}$. For any $(z, t) \in \mathcal{O} \times \mathbb{R}_{+}$, the function $s \rightarrow \Psi_{\delta}(z, t, s)$ belongs to $C_{\#}\left(\mathbb{R}_{s}\right)$ and if $T$ is such that supp $\psi \subset\left[0, T\left[\times \mathbb{R}^{2} \times \mathbb{R}^{2}\right.\right.$, one gets

$$
\begin{aligned}
\int_{\mathcal{O}} \int_{\mathbb{R}_{+}}\left\|\Psi_{\delta}(z, t)\right\|_{C_{\#}\left(\mathbb{R}_{s}\right)} \mathrm{d} t \mathrm{~d} z & \leq \frac{1}{4 \pi \delta}\left\|\nabla_{\widetilde{x}, \widetilde{v}} \psi\right\|_{L^{\infty}} \sqrt{1+\omega_{c}^{-2}} \int_{0}^{T} \int_{\mathcal{O}} \tilde{f}(t, \widetilde{x}, \widetilde{v}) \tilde{f}(t, \widetilde{y}, \widetilde{w}) \mathrm{d} z \mathrm{~d} t \\
& \leq \frac{T}{4 \pi \delta}\left\|\nabla_{\widetilde{x}, \widetilde{v}} \psi\right\|_{L^{\infty}} \sqrt{1+\omega_{c}^{-2}}\left\|f^{\mathrm{in}}\right\|_{L^{1}\left(\mathbb{R}^{2} \times \mathbb{R}^{2}\right)}^{2}<+\infty .
\end{aligned}
$$

The conclusion follows by Proposition 3.2.

We need to pass to the limit, when $\delta \searrow 0$, in (41). We introduce the notations

$$
T_{k, \delta}^{4}=\int_{\mathcal{O}} \int_{\mathbb{R}_{+}} \Psi_{\delta}\left(z, t, t / \varepsilon_{k}\right) \mathrm{d} t \mathrm{~d} z, \quad T_{\delta}^{4}=\frac{1}{T_{c}} \int_{\mathcal{O}} \int_{\mathbb{R}_{+}} \int_{0}^{T_{c}} \Psi_{\delta}(z, t, s) \mathrm{d} s \mathrm{~d} t \mathrm{~d} z
$$

and

$$
T^{4}=\frac{1}{T_{c}} \int_{\mathcal{O}} \int_{\mathbb{R}_{+}} \int_{0}^{T_{c}} \Psi(z, t, s) \mathrm{d} s \mathrm{~d} t \mathrm{~d} z
$$

We already know that

$$
\lim _{k \rightarrow+\infty} T_{k, \delta}^{4}=T_{\delta}^{4}, \text { for any } \delta>0
$$

and we claim that

$$
\lim _{k \rightarrow+\infty} T_{k}^{4}=T^{4} .
$$

We are done if we prove that $\lim _{\delta \searrow 0} T_{\delta}^{4}=T^{4}$ and

$$
\lim _{\delta \searrow 0} \sup _{k \in \mathbb{N}}\left|T_{k, \delta}^{4}-T_{k}^{4}\right|=0 .
$$

Observe that $\left|\Psi_{\delta}(z, t, s)\right| \leq|\Psi(z, t, s)|$ and $\lim _{\delta \searrow 0} \Psi_{\delta}(z, t, s)=\Psi(z, t, s),(z, t, s) \in \mathcal{O} \times \mathbb{R}_{+} \times$ $\mathbb{R}_{s}, \delta>0$. Therefore the convergence of $\left(T_{\delta}^{4}\right)_{\delta}$ toward $T^{4}$ comes by the dominated convergence theorem, provided that $\Psi \in L^{1}\left(\mathcal{O} \times \mathbb{R}_{+} \times\left[0, T_{c}\right]\right)$. Indeed, after change of variable one gets

$$
\begin{aligned}
\frac{1}{T_{c}} \int_{\mathcal{O}} \int_{\mathbb{R}_{+}} \int_{0}^{T_{c}}|\Psi(z, t, s)| \mathrm{d} s \mathrm{~d} t \mathrm{~d} z & \leq \frac{1}{T_{c}} \int_{0}^{T_{c}} \int_{0}^{T} \int_{\mathcal{O}} \tilde{f}\left(t, x+\frac{{ }^{\perp} v}{\omega_{c}}, \mathcal{R}\left(\omega_{c} s\right) v\right) \tilde{f}\left(t, y+\frac{{ }^{\perp} w}{\omega_{c}}, \mathcal{R}\left(\omega_{c} s\right) w\right) \\
& \times \frac{\|\nabla \psi\|_{L^{\infty}}}{2 \pi|x-y|} \sqrt{1+\omega_{c}^{-2}} \mathrm{~d} z \mathrm{~d} t \mathrm{~d} s .
\end{aligned}
$$

We are done if we show that the application

$$
(x, v, t, s) \rightarrow \int_{\mathbb{R}^{2}} \int_{\mathbb{R}^{2}} \frac{1}{|x-y|} \tilde{f}\left(t, y+\frac{{ }^{\perp} w}{\omega_{c}}, \mathcal{R}\left(\omega_{c} s\right) w\right) \mathrm{d} w \mathrm{~d} y
$$

is bounded on $\mathbb{R}^{2} \times \mathbb{R}^{2} \times[0, T] \times\left[0, T_{c}\right]$. By Remark 3.2 we know that for any $(y, w, t, s) \in$ $\mathbb{R}^{2} \times \mathbb{R}^{2} \times[0, T] \times\left[0, T_{c}\right]$ we have

$$
\tilde{f}\left(t, y+\frac{{ }^{\perp} w}{\omega_{c}}, \mathcal{R}\left(\omega_{c} s\right) w\right) \leq F\left(C_{T},|w|\right), \quad C_{T}=T \sup _{k}\left\|E^{\varepsilon_{k}}\right\|_{L^{\infty}\left([0, T] \times \mathbb{R}^{2}\right)}
$$


and therefore

$$
\begin{aligned}
\int_{\mathbb{R}^{2}} \int_{\mathbb{R}^{2}} \frac{1}{|x-y|} \tilde{f} & \left(t, y+\frac{{ }^{\perp} w}{\omega_{c}}, \mathcal{R}\left(\omega_{c} s\right) w\right) \mathrm{d} w \mathrm{~d} y \leq \int_{|x-y| \leq 1} \frac{1}{|x-y|} \int_{\mathbb{R}^{2}} F\left(C_{T},|w|\right) \mathrm{d} w \mathrm{~d} y \\
& +\int_{|x-y|>1} \frac{1}{|x-y|} \int_{\mathbb{R}^{2}} \tilde{f}\left(t, y+\frac{{ }^{\perp} w}{\omega_{c}}, \mathcal{R}\left(\omega_{c} s\right) w\right) \mathrm{d} w \mathrm{~d} y \\
& \leq 4 \pi^{2} \int_{0}^{+\infty} F\left(C_{T}, r\right) r \mathrm{~d} r+\int_{\mathbb{R}^{2}} \int_{\mathbb{R}^{2}} f^{\mathrm{in}}(y, w) \mathrm{d} w \mathrm{~d} y<+\infty .
\end{aligned}
$$

We concentrate now to the uniform convergence (43). Assume that $\operatorname{supp} \psi \subset\left[0, T\left[\times B_{R}\right.\right.$. Observe that

$$
\left|\nabla e_{\delta}-\nabla e\right|=\frac{\delta^{2}}{2 \pi|z|\left(|z|^{2}+\delta^{2}\right)} \leq \frac{1}{2 \pi|z|}, \quad z \in \mathbb{R}^{2} \backslash\{0\}, \quad \delta>0
$$

which implies (here $\left.R^{\prime}=R\left(1+1 /\left|\omega_{c}\right|\right)\right)$

$$
\begin{aligned}
\left|T_{k, \delta}^{4}-T_{k}^{4}\right| & \leq \int_{0}^{T} \int_{|x| \leq R^{\prime}} \int_{|v| \leq R} \int_{\mathbb{R}^{2}} \int_{\mathbb{R}^{2}} \tilde{f}\left(t, x+\frac{{ }^{\perp} v}{\omega_{c}}, \mathcal{R}\left(\omega_{c} t / \varepsilon_{k}\right) v\right) \tilde{f}\left(t, y+\frac{{ }^{\perp} w}{\omega_{c}}, \mathcal{R}\left(\omega_{c} t / \varepsilon_{k}\right) w\right) \\
& \times \frac{\|\nabla \psi\|_{L^{\infty}}}{2 \pi|x-y|} \frac{\delta^{2}}{|x-y|^{2}+\delta^{2}} \sqrt{1+\omega_{c}^{-2}} \mathrm{~d} w \mathrm{~d} y \mathrm{~d} v \mathrm{~d} x \mathrm{~d} t \\
& \leq \int_{0}^{T} \int_{|x| \leq R^{\prime}} \int_{|v| \leq R} \int_{\mathbb{R}^{2}} \int_{\mathbb{R}^{2}} F\left(C_{T},|v|\right) F\left(C_{T},|w|\right) \\
& \times \frac{\|\nabla \psi\|_{L^{\infty}}}{2 \pi|x-y|} \frac{\delta^{2}}{|x-y|^{2}+\delta^{2}} \sqrt{1+\omega_{c}^{-2}} \mathrm{~d} w \mathrm{~d} y \mathrm{~d} v \mathrm{~d} x \mathrm{~d} t .
\end{aligned}
$$

It is enough to check that $I_{\delta}:=\int_{|x| \leq R^{\prime}} \int_{\mathbb{R}^{2}} \frac{1}{2 \pi|x-y|} \frac{\delta^{2}}{|x-y|^{2}+\delta^{2}} \mathrm{~d} y \mathrm{~d} x \rightarrow 0$ as $\delta \searrow 0$. Indeed we have

$$
I_{\delta}=\int_{|x| \leq R^{\prime}} \int_{\mathbb{R}^{2}} \frac{1}{2 \pi|z|} \frac{\delta^{2}}{|z|^{2}+\delta^{2}} \mathrm{~d} z \mathrm{~d} x=\frac{\pi^{2}}{2}\left(R^{\prime}\right)^{2} \delta \rightarrow 0, \quad \text { as } \delta \searrow 0
$$


and thus the convergence in (42) holds true. Finally we determined the limit of the non linear term in the weak formulation (39)

$$
\begin{aligned}
& \lim _{k \rightarrow+\infty} \int_{\mathbb{R}_{+}} \int_{\mathbb{R}^{2}} \int_{\mathbb{R}^{2}} \tilde{f}^{\varepsilon_{k}}(t, \widetilde{x}, \widetilde{v})\left[\omega_{c}^{-1}{ }^{\perp} \nabla_{x} \phi^{\varepsilon_{k}}(t, x) \cdot \nabla_{\widetilde{x}} \psi+\mathcal{R}\left(\omega_{c} t / \varepsilon_{k}\right) \nabla_{x} \phi^{\varepsilon_{k}}(t, x) \cdot \nabla_{\widetilde{v}} \psi\right] \mathrm{d} \tilde{v} \mathrm{~d} \tilde{x} \mathrm{~d} t \\
& =\lim _{k \rightarrow+\infty}\left(T_{k}^{1}+T_{k}^{2}\right)=\lim _{k \rightarrow+\infty}\left(T_{k}^{1}+T_{k}^{3}+T_{k}^{4}\right)=T^{4} \\
& =\int_{\mathbb{R}_{+}} \int_{\mathbb{R}^{2}} \int_{\mathbb{R}^{2}} \tilde{f}(t, \widetilde{x}, \widetilde{v}) \omega_{c}^{-1} \nabla_{\widetilde{x}} \psi \cdot{ }^{\perp} \nabla_{\widetilde{x}} \int_{\mathbb{R}^{2}} \int_{\mathbb{R}^{2}} \tilde{f}(t, \widetilde{y}, \widetilde{w}) \\
& \times \frac{1}{T_{c}} \int_{0}^{T_{c}} e\left(\widetilde{x}-\widetilde{y}-\omega_{c}^{-1} \mathcal{R}\left(-\omega_{c} s\right)^{\perp}(\widetilde{v}-\widetilde{w})\right) \mathrm{d} s \mathrm{~d} \tilde{w} \mathrm{~d} \tilde{y} \mathrm{~d} \tilde{v} \mathrm{~d} \tilde{x} \mathrm{~d} t \\
& -\int_{\mathbb{R}_{+}} \int_{\mathbb{R}^{2}} \int_{\mathbb{R}^{2}} \tilde{f}(t, \widetilde{x}, \widetilde{v}) \omega_{c} \nabla_{\widetilde{v}} \psi \cdot{ }^{\perp} \nabla_{\widetilde{v}} \int_{\mathbb{R}^{2}} \int_{\mathbb{R}^{2}} \tilde{f}(t, \tilde{y}, \widetilde{w}) \\
& \times \frac{1}{T_{c}} \int_{0}^{T_{c}} e\left(\widetilde{x}-\widetilde{y}-\omega_{c}^{-1} \mathcal{R}\left(-\omega_{c} s\right)^{\perp}(\widetilde{v}-\widetilde{w})\right) \mathrm{d} s \mathrm{~d} \tilde{w} \mathrm{~d} \tilde{y} \mathrm{~d} \tilde{v} \mathrm{~d} \tilde{x} \mathrm{~d} t \\
& =\int_{\mathbb{R}_{+}} \int_{\mathbb{R}^{2}} \int_{\mathbb{R}^{2}} \tilde{f}(t, \widetilde{x}, \widetilde{v}) \omega_{c}^{-1} \nabla_{\widetilde{x}} \psi \cdot{ }^{\perp} \nabla_{\widetilde{x}} \int_{\mathbb{R}^{2}} \int_{\mathbb{R}^{2}} \tilde{f}(t, \widetilde{y}, \widetilde{w}) \mathcal{E}(\widetilde{x}-\widetilde{y}, \widetilde{v}-\widetilde{w}) \mathrm{d} \tilde{w} \mathrm{~d} \tilde{y} \mathrm{~d} \tilde{v} \mathrm{~d} \tilde{x} \mathrm{~d} t \\
& -\int_{\mathbb{R}_{+}} \int_{\mathbb{R}^{2}} \int_{\mathbb{R}^{2}} \tilde{f}(t, \widetilde{x}, \widetilde{v}) \omega_{c} \nabla_{\widetilde{v}} \psi \cdot{ }^{\perp} \nabla_{\widetilde{v}} \int_{\mathbb{R}^{2}} \int_{\mathbb{R}^{2}} \tilde{f}(t, \widetilde{y}, \widetilde{w}) \mathcal{E}(\widetilde{x}-\widetilde{y}, \widetilde{v}-\widetilde{w}) \mathrm{d} \tilde{w} \mathrm{~d} \tilde{y} \mathrm{~d} \tilde{v} \mathrm{~d} \tilde{x} \mathrm{~d} t \\
& =-\int_{\mathbb{R}_{+}} \int_{\mathbb{R}^{2}} \int_{\mathbb{R}^{2}} \tilde{f}(t, \widetilde{x}, \widetilde{v})\left[\mathcal{V}[\tilde{f}(t)](\widetilde{x}, \widetilde{v}) \cdot \nabla_{\widetilde{x}} \psi+\mathcal{A}[\tilde{f}(t)](\widetilde{x}, \widetilde{v}) \cdot \nabla_{\widetilde{v}} \psi\right] \mathrm{d} \tilde{v} \mathrm{~d} \tilde{x} \mathrm{~d} t .
\end{aligned}
$$

The limit formulation (39), as $k \rightarrow+\infty$, becomes

$$
\begin{aligned}
& -\int_{\mathbb{R}_{+}} \int_{\mathbb{R}^{2}} \int_{\mathbb{R}^{2}} \tilde{f} \partial_{t} \psi \mathrm{d} \tilde{v} \mathrm{~d} \tilde{x} \mathrm{~d} t-\int_{\mathbb{R}^{2}} \int_{\mathbb{R}^{2}} f^{\text {in }}\left(\tilde{x}-\frac{\perp}{\omega_{c}}, \widetilde{v}\right) \psi(0, \widetilde{x}, \widetilde{v}) \mathrm{d} \tilde{v} \mathrm{~d} \tilde{x} \\
& =\int_{\mathbb{R}_{+}} \int_{\mathbb{R}^{2}} \int_{\mathbb{R}^{2}} \tilde{f}\left[\mathcal{V}[\tilde{f}(t)](\widetilde{x}, \widetilde{v}) \cdot \nabla_{\widetilde{x}} \psi+\mathcal{A}[\tilde{f}(t)](\widetilde{x}, \widetilde{v}) \cdot \nabla_{\widetilde{v}} \psi\right] \mathrm{d} \tilde{v} \mathrm{~d} \tilde{x} \mathrm{~d} t, \quad \psi \in C_{c}^{2}\left(\mathbb{R}_{+} \times \mathbb{R}^{2} \times \mathbb{R}^{2}\right) .
\end{aligned}
$$

Actually, the velocity and acceleration fields $\mathcal{V}[\tilde{f}], \mathcal{A}[\tilde{f}]$ belong to $L^{\infty}\left([0, T] \times \mathbb{R}^{2} \times \mathbb{R}^{2}\right)$, for any $T \in \mathbb{R}_{+}$(cf. (46), (47)) and we deduce, by density arguments, that the above formulation holds true for any $\psi \in C_{c}^{1}\left(\mathbb{R}_{+} \times \mathbb{R}^{2} \times \mathbb{R}^{2}\right)$, saying that the density $\tilde{f}$ is a weak solution for the problem

$$
\begin{gathered}
\partial_{t} \tilde{f}+\mathcal{V}[\tilde{f}(t)] \cdot \nabla_{\widetilde{x}} \tilde{f}+\mathcal{A}[\tilde{f}(t)] \cdot \nabla_{\widetilde{v}} \tilde{f}=0, \quad(t, \widetilde{x}, \widetilde{v}) \in \mathbb{R}_{+} \times \mathbb{R}^{2} \times \mathbb{R}^{2} \\
\tilde{f}(0, \widetilde{x}, \widetilde{v})=f^{\text {in }}\left(\widetilde{x}-\frac{{ }^{\perp} \widetilde{v}}{\omega_{c}}, \widetilde{v}\right), \quad(\widetilde{x}, \widetilde{v}) \in \mathbb{R}^{2} \times \mathbb{R}^{2}
\end{gathered}
$$

where

$$
\mathcal{V}[\tilde{f}(t)]=-\omega_{c}^{-1}{ }^{\perp} \nabla_{\widetilde{x}} \tilde{\phi}[\tilde{f}(t)], \quad \mathcal{A}[\tilde{f}(t)]=\omega_{c}{ }^{\perp} \nabla_{\widetilde{v}} \tilde{\phi}[\tilde{f}(t)]
$$

and

$$
\tilde{\phi}[\tilde{f}(t)](\widetilde{x}, \widetilde{v})=\int_{\mathbb{R}^{2}} \int_{\mathbb{R}^{2}} \mathcal{E}(\widetilde{x}-\widetilde{y}, \widetilde{v}-\widetilde{w}) \tilde{f}(t, \widetilde{y}, \widetilde{w}) \mathrm{d} \tilde{w} \mathrm{~d} \tilde{y} .
$$

It remains to establish the strong convergence of $\left(\tilde{f}^{\varepsilon_{k}}\right)_{k}$ in $L^{2}\left([0, T] ; L^{2}\left(\mathbb{R}^{2} \times \mathbb{R}^{2}\right)\right)$ for any $T \in \mathbb{R}_{+}$. We already know that $\left(\tilde{f}^{\varepsilon_{k}}\right)_{k}$ converges weakly $\star$ in $L^{\infty}\left(\mathbb{R}_{+} ; L^{2}\left(\mathbb{R}^{2} \times \mathbb{R}^{2}\right)\right)$ toward $\tilde{f}$ 
and therefore $\left(\left.\tilde{f}^{\varepsilon_{k}}\right|_{[0, T]}\right)_{k}$ converges weakly in $L^{2}\left([0, T] ; L^{2}\left(\mathbb{R}^{2} \times \mathbb{R}^{2}\right)\right)$ toward $\left.\tilde{f}\right|_{[0, T]}$. We are done if we prove that

$$
\int_{\mathbb{R}^{2}} \int_{\mathbb{R}^{2}}(\tilde{f}(t, \tilde{x}, \widetilde{v}))^{2} \mathrm{~d} \tilde{v} \mathrm{~d} \tilde{x}=\int_{\mathbb{R}^{2}} \int_{\mathbb{R}^{2}}(\tilde{f}(0, \tilde{x}, \tilde{v}))^{2} \mathrm{~d} \tilde{v} \mathrm{~d} \tilde{x}, \quad t \in[0, T]
$$

since in that case we have

$$
\int_{0}^{T} \int_{\mathbb{R}^{2}} \int_{\mathbb{R}^{2}}(\tilde{f})^{2} \mathrm{~d} \tilde{v} \mathrm{~d} \tilde{x} \mathrm{~d} t=T \int_{\mathbb{R}^{2}} \int_{\mathbb{R}^{2}}\left(f^{\text {in }}\left(\widetilde{x}-\frac{{ }^{\perp}}{\omega_{c}}, \tilde{v}\right)\right)^{2} \mathrm{~d} \tilde{v} \mathrm{~d} \tilde{x}=\int_{0}^{T} \int_{\mathbb{R}^{2}} \int_{\mathbb{R}^{2}}\left(\tilde{f}^{\varepsilon_{k}}\right)^{2} \mathrm{~d} \tilde{v} \mathrm{~d} \tilde{x} \mathrm{~d} t
$$

saying that the weak convergence in $L^{2}\left([0, T] ; L^{2}\left(\mathbb{R}^{2} \times \mathbb{R}^{2}\right)\right)$ becomes strong. The conservation of the $L^{2}$ norm of $(\tilde{f}(t))_{t \in \mathbb{R}_{+}}$is a consequence of the equality

$$
\partial_{t} \frac{(\tilde{f})^{2}}{2}+\mathcal{V}[\tilde{f}(t)] \cdot \nabla_{\widetilde{x}} \frac{(\tilde{f})^{2}}{2}+\mathcal{A}[\tilde{f}(t)] \cdot \nabla_{\tilde{v}} \frac{(\tilde{f})^{2}}{2}=0 \text { in } \mathcal{D}^{\prime}\left(\mathbb{R}_{+} \times \mathbb{R}^{2} \times \mathbb{R}^{2}\right)
$$

which holds true provided that $\mathcal{V}[\tilde{f}], \mathcal{A}[\tilde{f}]$ are smooth, for example if $\mathcal{V}[\tilde{f}], \mathcal{A}[\tilde{f}]$ belongs to $L^{\infty}\left([0, T] ; H^{1}\left(B_{R}\right)\right), T, R \in \mathbb{R}_{+}$cf. [9]. Indeed, we have by Proposition 2.2

$\nabla_{\widetilde{x}} \tilde{\phi}[\tilde{f}(t)](\widetilde{x}, \widetilde{v})=\nabla_{\widetilde{x}}(\mathcal{E} \star \tilde{f}(t))=\nabla_{\xi} \mathcal{E} \star \tilde{f}(t)=-\int_{\mathbb{R}^{2}} \int_{\mathbb{R}^{2}} \frac{\widetilde{x}-\widetilde{y}}{2 \pi|\widetilde{x}-\widetilde{y}|^{2}} \tilde{f}(t, \widetilde{y}, \widetilde{w}) \mathbf{1}_{\left\{|\widetilde{x}-\widetilde{y}|>\frac{|\widetilde{v}-\widetilde{w}|}{\left|\omega_{c}\right|}\right\}} \mathrm{d} \tilde{w} \mathrm{~d} \tilde{y}$ and

$\nabla_{\widetilde{v}} \tilde{\phi}[\tilde{f}(t)](\widetilde{x}, \widetilde{v})=\nabla_{\widetilde{v}}(\mathcal{E} \star \tilde{f}(t))=\nabla_{\eta} \mathcal{E} \star \tilde{f}(t)=-\int_{\mathbb{R}^{2}} \int_{\mathbb{R}^{2}} \frac{\tilde{v}-\widetilde{w}}{2 \pi|\widetilde{v}-\widetilde{w}|^{2}} \tilde{f}(t, \widetilde{y}, \widetilde{w}) \mathbf{1}_{\left\{|\widetilde{x}-\widetilde{y}| \leq \frac{|\widetilde{v}-\widetilde{w}|}{|\omega c|}\right\}} \mathrm{d} \tilde{w} \mathrm{~d} \tilde{y}$.

Thanks to the inequality $\tilde{f}(t, \widetilde{y}, \widetilde{w}) \leq F\left(C_{T},|\widetilde{w}|\right),(t, \widetilde{y}, \widetilde{w}) \in[0, T] \times \mathbb{R}^{2} \times \mathbb{R}^{2}$, we obtain

$$
\begin{aligned}
\left|\omega_{c} \mathcal{V}([\tilde{f}(t)])\right| & =\left|\nabla_{\widetilde{x}} \tilde{\phi}[\tilde{f}(t)]\right| \leq \int_{\mathbb{R}^{2}} \int_{\mathbb{R}^{2}} \frac{\tilde{f}(t, \widetilde{y}, \widetilde{w})}{2 \pi|\widetilde{x}-\widetilde{y}|} \mathrm{d} \tilde{w} \mathrm{~d} \tilde{y} \\
& \leq \int_{\mathbb{R}^{2}} \int_{\mathbb{R}^{2}} \frac{\mathbf{1}_{\{|\widetilde{x}-\widetilde{y}| \leq 1\}}}{2 \pi|\widetilde{x}-\widetilde{y}|} \tilde{f}(t, \widetilde{y}, \widetilde{w}) \mathrm{d} \tilde{w} \mathrm{~d} \tilde{y}+\int_{\mathbb{R}^{2}} \int_{\mathbb{R}^{2}} \frac{\mathbf{1}_{\{|\widetilde{x}-\widetilde{y}|>1\}} \tilde{f}(t, \widetilde{y}, \widetilde{w}) \mathrm{d} \tilde{w} \mathrm{~d} \tilde{y}}{2 \pi|\widetilde{x}-\widetilde{y}|} \\
& \leq \int_{\mathbb{R}^{2}} \int_{\mathbb{R}^{2}} \frac{\mathbf{1}_{\{|\widetilde{x}-\widetilde{y}| \leq 1\}}}{2 \pi|\widetilde{x}-\widetilde{y}|} F\left(C_{T},|\widetilde{w}|\right) \mathrm{d} \tilde{w} \mathrm{~d} \tilde{y}+\int_{\mathbb{R}^{2}} \int_{\mathbb{R}^{2}} \frac{\tilde{f}(t, \widetilde{y}, \widetilde{w})}{2 \pi} \mathrm{d} \tilde{w} \mathrm{~d} \tilde{y} \\
& =\left\|F\left(C_{T},|\cdot|\right)\right\|_{L^{1}\left(\mathbb{R}^{2}\right)}+\frac{1}{2 \pi}\left\|f^{\mathrm{in}}\right\|_{L^{1}\left(\mathbb{R}^{2} \times \mathbb{R}^{2}\right)}<+\infty, \quad(t, \widetilde{x}, \widetilde{v}) \in[0, T] \times \mathbb{R}^{2} \times \mathbb{R}^{2}
\end{aligned}
$$

and similarly

$$
\begin{aligned}
\left|\omega_{c}^{-1} \mathcal{A}([\tilde{f}(t)])\right| & =\left|\nabla_{\widetilde{v}} \tilde{\phi}[\tilde{f}(t)]\right| \leq \int_{\mathbb{R}^{2}} \int_{\mathbb{R}^{2}} \frac{\mathbf{1}_{\left\{|\widetilde{x}-\widetilde{y}| \leq|\widetilde{v}-\widetilde{w}| /\left|\omega_{c}\right|\right\}} \tilde{f}(t, \widetilde{y}, \widetilde{w}) \mathrm{d} \tilde{w} \mathrm{~d} \tilde{y}}{} \\
& =\int_{|\widetilde{v}-\widetilde{w}| \leq 1} \frac{1}{2 \pi|\widetilde{v}-\widetilde{w}|} \int_{|\widetilde{x}-\widetilde{y}| \leq \frac{|\widetilde{v}-\widetilde{w}|}{\left|\omega_{c}\right|} \tilde{f}(t, \widetilde{y}, \widetilde{w}) \mathrm{d} \widetilde{y} \mathrm{~d} \widetilde{w}} \\
& +\int_{|\widetilde{v}-\widetilde{w}|>1} \frac{1}{2 \pi|\widetilde{v}-\widetilde{w}|} \int_{|\widetilde{x}-\widetilde{y}| \leq \frac{|\widetilde{v}-\widetilde{w}|}{\left|\omega_{c}\right|}} \tilde{f}(t, \widetilde{y}, \widetilde{w}) \mathrm{d} \widetilde{y} \mathrm{~d} \widetilde{w} \\
& \leq \int_{|\widetilde{v}-\widetilde{w}| \leq 1} \frac{1}{2 \pi|\widetilde{v}-\widetilde{w}|} \pi\left\|F^{\mathrm{in}}\right\|_{L^{\infty}} \frac{|\widetilde{v}-\widetilde{w}|^{2}}{\omega_{c}^{2}} \mathrm{~d} \widetilde{w}+\frac{1}{2 \pi}\left\|f^{\mathrm{in}}\right\|_{L^{1}\left(\mathbb{R}^{2} \times \mathbb{R}^{2}\right)} \\
& =\frac{\pi\left\|F^{\mathrm{in}}\right\|_{L^{\infty}}}{3 \omega_{c}^{2}}+\frac{1}{2 \pi}\left\|f^{\mathrm{in}}\right\|_{L^{1}\left(\mathbb{R}^{2} \times \mathbb{R}^{2}\right)}<+\infty, \quad(t, \widetilde{x}, \widetilde{v}) \in[0, T] \times \mathbb{R}^{2} \times \mathbb{R}^{2} .
\end{aligned}
$$


The previous computations say that the velocity and acceleration fields $\mathcal{V}[\tilde{f}], \mathcal{A}[\tilde{f}]$ in (45) belong to $L^{\infty}\left([0, T] \times \mathbb{R}^{2} \times \mathbb{R}^{2}\right)$, for any $T \in \mathbb{R}_{+}$, and thus $\frac{(\tilde{f})^{2}}{2}$ will propagate with finite speed (once that we have established (45)). Let us estimate now the velocity and acceleration fields in $L^{\infty}\left([0, T] ; H^{1}\left(B_{R}\right)\right)$. Clearly $\mathcal{V}[\tilde{f}], \mathcal{A}[\tilde{f}] \in L^{\infty}\left([0, T] ; L^{2}\left(B_{R}\right)\right)$ and we need to estimate the second derivatives of $\tilde{\phi}[\tilde{f}]$ in $L^{\infty}\left([0, T] ; L^{2}\left(B_{R}\right)\right)$. Thanks to Proposition 2.2 we obtain

$$
\begin{aligned}
\partial_{\widetilde{x}}^{2} \tilde{\phi}[\tilde{f}(t)] & =\partial_{\widetilde{x}}^{2}(\mathcal{E} \star \tilde{f}(t))=\partial_{\xi}^{2} \mathcal{E} \star \tilde{f}(t) \\
& =-\int_{\mathbb{R}^{2}} \int_{\mathbb{R}^{2}}\left(I_{2}-2 \frac{(\widetilde{x}-\widetilde{y}) \otimes(\widetilde{x}-\widetilde{y})}{|\widetilde{x}-\widetilde{y}|^{2}}\right) \frac{\tilde{f}(t, \widetilde{y}, \widetilde{w}) \mathbf{1}_{\left\{|\widetilde{x}-\widetilde{y}|>|\widetilde{v}-\widetilde{w}| /\left|\omega_{c}\right|\right\}}}{2 \pi|\widetilde{x}-\widetilde{y}|^{2}} \mathrm{~d} \tilde{w} \mathrm{~d} \tilde{y} \\
& -\int_{|\widetilde{x}-\widetilde{y}|=\frac{|\widetilde{v}-\widetilde{w}|}{\left|\omega_{c}\right|}} \frac{(\widetilde{x}-\widetilde{y}) \otimes(\widetilde{x}-\widetilde{y})}{|\widetilde{x}-\widetilde{y}|^{2}} \frac{\tilde{f}(t, \widetilde{y}, \widetilde{w})}{2 \pi|\widetilde{x}-\widetilde{y}| \sqrt{1+\omega_{c}^{-2}}} \mathrm{~d} \sigma(\widetilde{y}, \widetilde{w}) .
\end{aligned}
$$

We are done if we show that

$$
I_{1}(t, \widetilde{x}, \widetilde{v}):=\int_{\mathbb{R}^{2}} \int_{|\widetilde{x}-\widetilde{y}|>\frac{|\widetilde{v}-\widetilde{w}|}{\left|\omega_{c}\right|}} \frac{\tilde{f}(t, \widetilde{y}, \widetilde{w})}{2 \pi|\widetilde{x}-\widetilde{y}|^{2}} \mathrm{~d} \widetilde{y} \mathrm{~d} \widetilde{w}
$$

and

$$
I_{2}(t, \widetilde{x}, \widetilde{v}):=\int_{\mathbb{R}^{2}} \int_{|\widetilde{x}-\widetilde{y}|=\frac{|\widetilde{v}-\widetilde{w}|}{\left|\omega_{c}\right|}} \frac{\tilde{f}(t, \widetilde{y}, \widetilde{w})}{2 \pi|\widetilde{x}-\widetilde{y}|} \mathrm{d} \sigma(\widetilde{y}) \mathrm{d} \widetilde{w}
$$

belong to $L^{\infty}\left([0, T] ; L^{2}\left(B_{R}\right)\right)$. For the first integral $I_{1}$ we write

$$
\begin{aligned}
I_{1}(t, \widetilde{x}, \widetilde{v}) & =\int_{\mathbb{R}^{2}} \int_{|\widetilde{x}-\widetilde{y}|>\max \left\{\frac{|\widetilde{v}-\widetilde{w}|}{\left|\omega_{c}\right|}, 1\right\}} \frac{\tilde{f}(t, \widetilde{y}, \widetilde{w})}{2 \pi|\widetilde{x}-\widetilde{y}|^{2}} \mathrm{~d} \widetilde{y} \mathrm{~d} \widetilde{w}+\int_{\mathbb{R}^{2}} \int_{1 \geq|\widetilde{x}-\widetilde{y}|>\frac{|\widetilde{v}-\widetilde{w}|}{\left|\omega_{c}\right|}} \frac{\tilde{f}(t, \widetilde{y}, \widetilde{w})}{2 \pi|\widetilde{x}-\widetilde{y}|^{2}} \mathrm{~d} \widetilde{y} \mathrm{~d} \widetilde{w} \\
& \leq \frac{1}{2 \pi}\left\|f^{\mathrm{in}}\right\|_{L^{1}\left(\mathbb{R}^{2} \times \mathbb{R}^{2}\right)}+\int_{|\widetilde{v}-\widetilde{w}|<\left|\omega_{c}\right|} F\left(C_{T},|\widetilde{w}|\right) \int_{1 \geq|\widetilde{x}-\widetilde{y}|>\frac{|\widetilde{v}-\widetilde{w}|}{\left|\omega_{c}\right|}} \frac{1}{2 \pi|\widetilde{x}-\widetilde{y}|^{2}} \mathrm{~d} \widetilde{y} \mathrm{~d} \widetilde{w} \\
& =\frac{1}{2 \pi}\left\|f^{\mathrm{in}}\right\|_{L^{1}\left(\mathbb{R}^{2} \times \mathbb{R}^{2}\right)}+\int_{|\widetilde{v}-\widetilde{w}|<\left|\omega_{c}\right|} F\left(C_{T},|\widetilde{w}|\right) \ln \frac{\left|\omega_{c}\right|}{|\widetilde{v}-\widetilde{w}|} \mathrm{d} \widetilde{w}=I_{1}^{\prime}+I_{2}^{\prime}
\end{aligned}
$$

and we observe that

$$
\begin{aligned}
\int_{B_{R}}\left(I_{2}^{\prime}\right)^{2} \mathrm{~d} \widetilde{v} \mathrm{~d} \widetilde{x} & \leq \int_{|\widetilde{x}| \leq R} \int_{|\widetilde{v}| \leq R}\left(\int_{|\widetilde{v}-\widetilde{w}|<\left|\omega_{c}\right|} F\left(C_{T},|\widetilde{w}|\right) \ln \frac{\left|\omega_{c}\right|}{|\widetilde{v}-\widetilde{w}|} \mathrm{d} \widetilde{w}\right)^{2} \mathrm{~d} \widetilde{v} \mathrm{~d} \widetilde{x} \\
& \leq \pi R^{2} \int_{|\widetilde{v}| \leq R}\left(\int_{|\widetilde{v}-\widetilde{w}|<\left|\omega_{c}\right|} F\left(C_{T},|\widetilde{w}|\right) \mathrm{d} \widetilde{w}\right)\left(\int_{|\widetilde{v}-\widetilde{w}|<\left|\omega_{c}\right|} F\left(C_{T},|\widetilde{w}|\right) \ln ^{2} \frac{\left|\omega_{c}\right|}{|\widetilde{v}-\widetilde{w}|} \mathrm{d} \widetilde{w}\right) \mathrm{d} \widetilde{v} \\
& \leq \pi R^{2}\left\|F\left(C_{T},|\cdot|\right)\right\|_{L^{1}\left(\mathbb{R}^{2}\right)} \int_{\mathbb{R}^{2}} F\left(C_{T},|\widetilde{w}|\right) \int_{|\widetilde{v}-\widetilde{w}|<\left|\omega_{c}\right|} \ln ^{2} \frac{\left|\omega_{c}\right|}{|\widetilde{v}-\widetilde{w}|} \mathrm{d} \widetilde{v} \mathrm{~d} \widetilde{w} \\
& =\pi R^{2}\left\|F\left(C_{T},|\cdot|\right)\right\|_{L^{1}\left(\mathbb{R}^{2}\right)}^{2} \omega_{c}^{2} \int_{|z| \leq 1} \ln ^{2}|z| \mathrm{d} z<+\infty .
\end{aligned}
$$

The second integral $I_{2}$ belongs to $L^{\infty}\left([0, T] \times \mathbb{R}^{2} \times \mathbb{R}^{2}\right)$, and thus to $L^{\infty}\left([0, T] ; L^{2}\left(B_{R}\right)\right)$

$$
I_{2}(t, \widetilde{x}, \widetilde{v}) \leq \int_{\mathbb{R}^{2}} F\left(C_{T},|\widetilde{w}|\right) \int_{|\widetilde{x}-\widetilde{y}|=\frac{|\widetilde{v}-\widetilde{w}|}{\left|\omega_{c}\right|}} \frac{\mathrm{d} \sigma(\widetilde{y})}{2 \pi|\widetilde{x}-\widetilde{y}|} \mathrm{d} \tilde{w}=\int_{\mathbb{R}^{2}} F\left(C_{T},|\widetilde{w}|\right) \mathrm{d} \tilde{w}<+\infty .
$$


Similarly, the second derivatives with respect to $\widetilde{v}$ are given by

$$
\begin{aligned}
\partial_{\widetilde{v}}^{2} \tilde{\phi}[\tilde{f}(t)] & =\partial_{\widetilde{v}}^{2}(\mathcal{E} \star \tilde{f}(t))=\partial_{\eta}^{2} \mathcal{E} \star \tilde{f}(t) \\
& =-\int_{\mathbb{R}^{2}} \int_{\mathbb{R}^{2}}\left(I_{2}-2 \frac{(\widetilde{v}-\widetilde{w}) \otimes(\widetilde{v}-\widetilde{w})}{|\widetilde{v}-\widetilde{w}|^{2}}\right) \frac{\tilde{f}(t, \widetilde{y}, \widetilde{w}) \mathbf{1}_{\left\{|\widetilde{x}-\widetilde{y}| \leq|\widetilde{v}-\widetilde{w}| /\left|\omega_{c}\right|\right\}}}{2 \pi|\widetilde{v}-\widetilde{w}|^{2}} \mathrm{~d} \tilde{w} \mathrm{~d} \tilde{y} \\
& -\int_{|\widetilde{x}-\widetilde{y}|=\frac{|\widetilde{v}-\widetilde{w}|}{\left|\omega_{c}\right|}} \frac{(\widetilde{v}-\widetilde{w}) \otimes(\widetilde{v}-\widetilde{w})}{|\widetilde{v}-\widetilde{w}|^{2}} \frac{\tilde{f}(t, \widetilde{y}, \widetilde{w})}{2 \pi|\widetilde{v}-\widetilde{w}| \sqrt{1+\omega_{c}^{2}}} \mathrm{~d} \sigma(\widetilde{y}, \widetilde{w})
\end{aligned}
$$

and we observe that

$$
\begin{aligned}
I_{3}(t, \widetilde{x}, \widetilde{v}) & :=\int_{\mathbb{R}^{2}} \int_{|\widetilde{x}-\widetilde{y}| \leq \frac{|\widetilde{v}-\widetilde{w}|}{\left|\omega_{c}\right|}} \frac{\tilde{f}(t, \widetilde{y}, \widetilde{w})}{2 \pi|\widetilde{v}-\widetilde{w}|^{2}} \mathrm{~d} \widetilde{y} \mathrm{~d} \widetilde{w} \\
& \leq \frac{1}{2 \omega_{c}^{2}} \int_{\mathbb{R}^{2}} F\left(C_{T},|\widetilde{w}|\right) \mathrm{d} \tilde{w}<+\infty, \quad(t, \widetilde{x}, \widetilde{v}) \in[0, T] \times \mathbb{R}^{2} \times \mathbb{R}^{2}
\end{aligned}
$$

and

$$
\begin{aligned}
I_{4}(t, \widetilde{x}, \widetilde{v}) & :=\int_{\mathbb{R}^{2}} \int_{|\widetilde{x}-\widetilde{y}|=\frac{|\widetilde{v}-\widetilde{w}|}{\left|\omega_{c}\right|}} \frac{\tilde{f}(t, \widetilde{y}, \widetilde{w})}{2 \pi|\widetilde{v}-\widetilde{w}|} \mathrm{d} \sigma(\widetilde{y}) \mathrm{d} \widetilde{w} \\
& \leq \frac{1}{\left|\omega_{c}\right|} \int_{\mathbb{R}^{2}} F\left(C_{T},|\widetilde{w}|\right) \mathrm{d} \tilde{w}<+\infty, \quad(t, \widetilde{x}, \widetilde{v}) \in[0, T] \times \mathbb{R}^{2} \times \mathbb{R}^{2} .
\end{aligned}
$$

Finally the second derivatives $\left(\nabla_{\widetilde{x}} \otimes \nabla_{\widetilde{v}}\right) \tilde{\phi}[\tilde{f}]$ are bounded as well. Indeed we have

$$
\begin{aligned}
\left(\nabla_{\widetilde{x}} \otimes \nabla_{\widetilde{v}}\right) \tilde{\phi}[\tilde{f}(t)] & =\left(\nabla_{\widetilde{x}} \otimes \nabla_{\widetilde{v}}\right)(\mathcal{E} \star \tilde{f}(t))=\left(\nabla_{\xi} \otimes \nabla_{\eta} \mathcal{E}\right) \star \tilde{f}(t) \\
& =\int_{|\widetilde{x}-\widetilde{y}|=\frac{|\widetilde{v}-\widetilde{w}|}{\left|\omega_{c}\right|}} \frac{(\widetilde{x}-\widetilde{y}) \otimes(\widetilde{v}-\widetilde{w})}{|\widetilde{x}-\widetilde{y}||\widetilde{v}-\widetilde{w}|} \frac{\tilde{f}(t, \widetilde{y}, \widetilde{w})}{2 \pi \omega_{c}|\widetilde{v}-\widetilde{w}|} \frac{\mathrm{d} \sigma(\widetilde{y}, \widetilde{w})}{\sqrt{1+\omega_{c}^{-2}}}
\end{aligned}
$$

and

$$
\begin{aligned}
I_{5}(t, \widetilde{x}, \widetilde{v}) & :=\int_{\mathbb{R}^{2}} \int_{|\widetilde{x}-\widetilde{y}|=\frac{|\widetilde{v}-\widetilde{w}|}{\left|\omega_{c}\right|}} \frac{\tilde{f}(t, \widetilde{y}, \widetilde{w})}{2 \pi|\widetilde{v}-\widetilde{w}|} \mathrm{d} \sigma(\widetilde{y}) \mathrm{d} \widetilde{w} \\
& \leq \frac{1}{\left|\omega_{c}\right|} \int_{\mathbb{R}^{2}} F\left(C_{T},|\widetilde{w}|\right) \mathrm{d} \tilde{w}<+\infty, \quad(t, \widetilde{x}, \widetilde{v}) \in[0, T] \times \mathbb{R}^{2} \times \mathbb{R}^{2} .
\end{aligned}
$$

The previous computations show that $\mathcal{V}[\tilde{f}], \mathcal{A}[\tilde{f}] \in L^{\infty}\left([0, T] \times \mathbb{R}^{2} \times \mathbb{R}^{2}\right) \cap L^{\infty}\left([0, T] ; H^{1}\left(B_{R}\right)\right)$ for any $T, R \in \mathbb{R}_{+}$. Therefore (45) holds true and the finite speed propagation property allows us to establish the conservation of the $L^{2}$ norm of $(\tilde{f}(t))_{t \in \mathbb{R}_{+}}$. We deduce that $\left(\tilde{f}^{\varepsilon_{k}}\right)_{k}$ converges strongly in $L^{2}\left([0, T] ; L^{2}\left(\mathbb{R}^{2} \times \mathbb{R}^{2}\right)\right)$ toward $\tilde{f}$, for any $T \in \mathbb{R}_{+}$.

Remark 3.5 Since $\mathcal{V}[\tilde{f}], \mathcal{A}[\tilde{f}]$ belong to $L^{\infty}\left([0, T] \times \mathbb{R}^{2} \times \mathbb{R}^{2}\right)$ for any $T \in \mathbb{R}_{+}$, the equation (44) propagates all moments in $(\widetilde{x}, \widetilde{v})$. Indeed, thanks to the inequalities

$$
\begin{aligned}
& \frac{\mathrm{d}}{\mathrm{d} t} \int_{\mathbb{R}^{2}} \int_{\mathbb{R}^{2}} \tilde{f}(t, \tilde{x}, \widetilde{v})|\widetilde{x}|^{m} \mathrm{~d} \tilde{v} \mathrm{~d} \tilde{x} \leq m \int_{\mathbb{R}^{2}} \int_{\mathbb{R}^{2}}|\mathcal{V}[\tilde{f}(t)]| \tilde{f}(t, \tilde{x}, \widetilde{v})|\widetilde{x}|^{m-1} \mathrm{~d} \tilde{v} \mathrm{~d} \tilde{x}, \quad m \in \mathbb{N}^{\star} \\
& \frac{\mathrm{d}}{\mathrm{d} t} \int_{\mathbb{R}^{2}} \int_{\mathbb{R}^{2}} \tilde{f}(t, \widetilde{x}, \widetilde{v})|\widetilde{v}|^{n} \mathrm{~d} \tilde{v} \mathrm{~d} \tilde{x} \leq n \int_{\mathbb{R}^{2}} \int_{\mathbb{R}^{2}}|\mathcal{A}[\tilde{f}(t)]| \tilde{f}(t, \widetilde{x}, \widetilde{v})|\widetilde{v}|^{n-1} \mathrm{~d} \tilde{v} \mathrm{~d} \tilde{x}, \quad n \in \mathbb{N}^{\star}
\end{aligned}
$$


it is easily seen, by recurrence, that

$$
\int_{\mathbb{R}^{2}} \int_{\mathbb{R}^{2}} \tilde{f}(\cdot, \widetilde{x}, \widetilde{v})|\widetilde{x}|^{m} \mathrm{~d} \tilde{v} \mathrm{~d} \tilde{x} \in L^{\infty}([0, T]), \quad \int_{\mathbb{R}^{2}} \int_{\mathbb{R}^{2}} \tilde{f}(\cdot, \widetilde{x}, \widetilde{v})|\widetilde{v}|^{n} \mathrm{~d} \tilde{v} \mathrm{~d} \tilde{x} \in L^{\infty}([0, T])
$$

provided that

$$
\int_{\mathbb{R}^{2}} \int_{\mathbb{R}^{2}} f^{\text {in }}\left(\widetilde{x}-\frac{\perp \widetilde{v}}{\omega_{c}}, \widetilde{v}\right)|\widetilde{x}|^{m} \mathrm{~d} \tilde{v} \mathrm{~d} \tilde{x}<+\infty, \int_{\mathbb{R}^{2}} \int_{\mathbb{R}^{2}} f^{\text {in }}\left(\widetilde{x}-\frac{\perp \widetilde{v}}{\omega_{c}}, \widetilde{v}\right)|\widetilde{v}|^{n} \mathrm{~d} \tilde{v} \mathrm{~d} \tilde{x}<+\infty .
$$

Remark 3.6 For any $s \in \mathbb{R}$ we consider the function $\rho_{s}: \mathbb{R}_{+} \times \mathbb{R}^{2} \times \mathbb{R}^{2} \rightarrow \mathbb{R}_{+}$given by

$$
\widetilde{\rho}_{s}(t, \widetilde{x}, \widetilde{v})=\int_{\mathbb{R}^{2}} \tilde{f}\left(t, \widetilde{x}-\omega_{c}^{-1} \mathcal{R}\left(-\omega_{c} s\right)^{\perp}(\widetilde{v}-\widetilde{w}), \widetilde{w}\right) \mathrm{d} \tilde{w}, \quad(t, \widetilde{x}, \widetilde{v}) \in \mathbb{R}_{+} \times \mathbb{R}^{2} \times \mathbb{R}^{2} .
$$

Observe that for any $(t, \widetilde{x}, \widetilde{v}) \in[0, T] \times \mathbb{R}^{2} \times \mathbb{R}^{2}$ we have

$$
0 \leq \widetilde{\rho}_{s}(t, \widetilde{x}, \widetilde{v}) \leq \int_{\mathbb{R}^{2}} F\left(C_{T},|\widetilde{w}|\right) \mathrm{d} \tilde{w}<+\infty
$$

saying that $\widetilde{\rho}_{s} \in L^{\infty}\left([0, T] \times \mathbb{R}^{2} \times \mathbb{R}^{2}\right)$, uniformly with respect to $s \in \mathbb{R}$. Notice also that for any $(t, \widetilde{v}) \in \mathbb{R}_{+} \times \mathbb{R}^{2}$ we have

$$
\int_{\mathbb{R}^{2}} \widetilde{\rho}_{s}(t, \widetilde{x}, \widetilde{v}) \mathrm{d} \tilde{x}=\int_{\mathbb{R}^{2}} \int_{\mathbb{R}^{2}} \tilde{f}\left(t, \widetilde{x}-\omega_{c}^{-1} \mathcal{R}\left(-\omega_{c} s\right)^{\perp}(\widetilde{v}-\widetilde{w}), \widetilde{w}\right) \mathrm{d} \widetilde{x} \mathrm{~d} \widetilde{w}=\left\|f^{\mathrm{in}}\right\|_{L^{1}\left(\mathbb{R}^{2} \times \mathbb{R}^{2}\right)} .
$$

Therefore the family $\left(\widetilde{\rho}_{s}\right)_{s}$ remains into a bounded set of $L^{\infty}\left([0, T] \times \mathbb{R}_{\tilde{v}}^{2} ; L^{1} \cap L^{\infty}\left(\mathbb{R}_{\widetilde{x}}^{2}\right)\right)$, for any $T \in \mathbb{R}_{+}$. We introduce the average charge density

$$
\widetilde{\rho}(t, \widetilde{x}, \widetilde{v})=\frac{1}{T_{c}} \int_{0}^{T_{c}} \widetilde{\rho}_{s}(t, \widetilde{x}, \widetilde{v}) \mathrm{d} s, \quad(t, \widetilde{x}, \widetilde{v}) \in \mathbb{R}_{+} \times \mathbb{R}^{2} \times \mathbb{R}^{2}
$$

which verifies

$$
0 \leq \widetilde{\rho}(t, \widetilde{x}, \widetilde{v}) \leq\left\|F\left(C_{T},|\cdot|\right)\right\|_{L^{1}\left(\mathbb{R}^{2}\right)}, \quad(t, \widetilde{x}, \widetilde{v}) \in[0, T] \times \mathbb{R}^{2} \times \mathbb{R}^{2}
$$

and

$$
\int_{\mathbb{R}^{2}} \widetilde{\rho}(t, \widetilde{x}, \widetilde{v}) \mathrm{d} \tilde{x}=\left\|f^{\mathrm{in}}\right\|_{L^{1}\left(\mathbb{R}^{2} \times \mathbb{R}^{2}\right)}, \quad(t, \widetilde{v}) \in \mathbb{R}_{+} \times \mathbb{R}^{2} .
$$

The definition of the function $\mathcal{E}$ allows us to write for any $(t, \widetilde{x}, \widetilde{v}) \in \mathbb{R}_{+} \times \mathbb{R}^{2} \times \mathbb{R}^{2}$

$$
\begin{aligned}
\tilde{\phi}[\tilde{f}(t)](\widetilde{x}, \widetilde{v}) & =\int_{\mathbb{R}^{2}} \int_{\mathbb{R}^{2}} \mathcal{E}(\widetilde{x}-\widetilde{y}, \widetilde{v}-\widetilde{w}) \tilde{f}(t, \widetilde{y}, \widetilde{w}) \mathrm{d} \tilde{w} \mathrm{~d} \tilde{y} \\
& =\frac{1}{T_{c}} \int_{0}^{T_{c}} \int_{\mathbb{R}^{2}} \int_{\mathbb{R}^{2}} e\left(\widetilde{x}-\widetilde{y}-\omega_{c}^{-1} \mathcal{R}\left(-\omega_{c} s\right)^{\perp}(\widetilde{v}-\widetilde{w})\right) \tilde{f}(t, \widetilde{y}, \widetilde{w}) \mathrm{d} \tilde{w} \mathrm{~d} \tilde{y} \mathrm{~d} s \\
& =\frac{1}{T_{c}} \int_{0}^{T_{c}} \int_{\mathbb{R}^{2}} \int_{\mathbb{R}^{2}} e(\widetilde{x}-\tilde{z}) \tilde{f}\left(t, \tilde{z}-\omega_{c}^{-1} \mathcal{R}\left(-\omega_{c} s\right)^{\perp}(\widetilde{v}-\widetilde{w}), \widetilde{w}\right) \mathrm{d} \widetilde{w} \mathrm{~d} \tilde{z} \mathrm{~d} s \\
& =\frac{1}{T_{c}} \int_{0}^{T_{c}} \int_{\mathbb{R}^{2}} e(\widetilde{x}-\tilde{z}) \widetilde{\rho}_{s}(t, \tilde{z}, \widetilde{v}) \mathrm{d} \tilde{z} \mathrm{~d} s \\
& =\int_{\mathbb{R}^{2}} e(\widetilde{x}-\tilde{z}) \widetilde{\rho}(t, \tilde{z}, \widetilde{v}) \mathrm{d} \tilde{z} \\
& =(e \star \widetilde{\rho}(t, \cdot, \widetilde{v}))(\widetilde{x}) .
\end{aligned}
$$


Therefore the function $\tilde{\phi}[\tilde{f}(t)]$ satisfies the Poisson equation (see also (24))

$$
-\Delta_{\widetilde{x}} \tilde{\phi}[\tilde{f}(t)]=\tilde{\rho}(t, \widetilde{x}, \widetilde{v})=\int_{\mathbb{R}^{2}} \frac{1}{T_{c}} \int_{0}^{T_{c}} \tilde{f}\left(t, \widetilde{x}-\omega_{c}^{-1} \mathcal{R}\left(-\omega_{c} s\right)^{\perp}(\widetilde{v}-\widetilde{w}), \widetilde{w}\right) \mathrm{d} s \mathrm{~d} \tilde{w} .
$$

If the initial density $f^{\text {in }}=f^{\text {in }}(x, v)$ is compactly supported in $\mathbb{R}^{2} \times \mathbb{R}^{2}$, we know by Remark 3.4 that for any $T \in \mathbb{R}_{+}$, the densities $\left.\tilde{f}^{\varepsilon_{k}}\right|_{[0, T] \times \mathbb{R}^{2} \times \mathbb{R}^{2}}$ remain compactly supported, uniformly with respect to $k \in \mathbb{N}$. By weak $\star$ convergence we obtain that $\left.\tilde{f}\right|_{[0, T] \times \mathbb{R}^{2} \times \mathbb{R}^{2}}$ is compactly supported and therefore $\widetilde{x} \rightarrow \widetilde{\rho}_{s}(t, \widetilde{x}, \widetilde{v})$ remain compactly supported, uniformly with respect to $s \in \mathbb{R}$ and $(t, \widetilde{v}) \in[0, T] \times\left\{\widetilde{v}^{\prime},\left|\widetilde{v}^{\prime}\right| \leq R\right\}$. We deduce that $\widetilde{x} \rightarrow \widetilde{\rho}(t, \widetilde{x}, \widetilde{v})$ remains compactly supported, uniformly with respect to $(t, \widetilde{v}) \in[0, T] \times\left\{\widetilde{v}^{\prime},\left|\widetilde{v}^{\prime}\right| \leq R\right\}$. As $\widetilde{\rho}$ is bounded on $[0, T] \times \mathbb{R}^{2} \times \mathbb{R}^{2}\left(\right.$ cf. (48)) we establish that $\tilde{\phi}[\tilde{f}(t)](\widetilde{x}, \widetilde{v})=\int_{\mathbb{R}^{2}} e(\widetilde{x}-\widetilde{z}) \widetilde{\rho}(t, \tilde{z}, \widetilde{v}) \mathrm{d} \tilde{z}$ is locally bounded on $[0, T] \times \mathbb{R}^{2} \times \mathbb{R}^{2}$.

We inquire now about the stability of the electric potentials

$$
\widetilde{\phi}^{\varepsilon_{k}}(t, \widetilde{x}, \widetilde{v})=\phi^{\varepsilon_{k}}\left(t, \widetilde{x}-\omega_{c}^{-1} \mathcal{R}\left(-\omega_{c} t / \varepsilon_{k}\right)^{\perp} \widetilde{v}\right)
$$

Proposition 3.4 Assume that the hypotheses of Theorem 1.1 hold true. Moreover suppose that the initial density $f^{\text {in }}$ has compact support in $\mathbb{R}^{2} \times \mathbb{R}^{2}$. Then we have the following convergences, as $k \rightarrow+\infty$, for any $\psi \in C_{c}^{0}\left(\mathbb{R}_{+} \times \mathbb{R}^{2} \times \mathbb{R}^{2}\right)$

$$
\begin{gathered}
\lim _{k \rightarrow+\infty} \int_{\mathbb{R}_{+}} \int_{\mathbb{R}^{2}} \int_{\mathbb{R}^{2}} \tilde{\phi}^{\varepsilon_{k}} \psi \mathrm{d} \tilde{v} \mathrm{~d} \tilde{x} \mathrm{~d} t=\int_{\mathbb{R}_{+}} \int_{\mathbb{R}^{2}} \int_{\mathbb{R}^{2}} \tilde{\phi} \psi \mathrm{d} \tilde{v} \mathrm{~d} \tilde{x} \mathrm{~d} t \\
\lim _{k \rightarrow+\infty}-\int_{\mathbb{R}_{+}} \int_{\mathbb{R}^{2}} \int_{\mathbb{R}^{2}} \omega_{c}^{-1}{ }^{\perp} \nabla_{\widetilde{x}} \widetilde{\phi}^{\varepsilon} \psi \mathrm{d} \tilde{v} \mathrm{~d} \tilde{x} \mathrm{~d} t=\int_{\mathbb{R}_{+}} \int_{\mathbb{R}^{2}} \int_{\mathbb{R}^{2}} \mathcal{V}[\tilde{f}(t)](\tilde{x}, \widetilde{v}) \psi \mathrm{d} \tilde{v} \mathrm{~d} \tilde{x} \mathrm{~d} t \\
\lim _{k \rightarrow+\infty} \int_{\mathbb{R}_{+}} \int_{\mathbb{R}^{2}} \int_{\mathbb{R}^{2}} \omega_{c}{ }^{\perp} \nabla_{\widetilde{v}} \widetilde{\phi}^{\varepsilon_{k}} \psi \mathrm{d} \tilde{v} \mathrm{~d} \tilde{x} \mathrm{~d} t=\int_{\mathbb{R}_{+}} \int_{\mathbb{R}^{2}} \int_{\mathbb{R}^{2}} \mathcal{A}[\tilde{f}(t)](\tilde{x}, \widetilde{v}) \psi \mathrm{d} \tilde{v} \mathrm{~d} \tilde{x} \mathrm{~d} t .
\end{gathered}
$$

Moreover, we have for any $\eta \in C_{c}^{0}\left(\mathbb{R}_{+}\right)$

$$
\lim _{k \rightarrow+\infty} \int_{\mathbb{R}_{+}} \eta(t) \int_{\mathbb{R}^{2}}\left|\nabla_{x} \phi^{\varepsilon_{k}}(t, x)\right|^{2} \mathrm{~d} x \mathrm{~d} t=\int_{\mathbb{R}_{+}} \eta(t) \int_{\mathbb{R}^{2}} \int_{\mathbb{R}^{2}} \tilde{f}(t, \widetilde{x}, \widetilde{v}) \tilde{\phi}[\tilde{f}(t)](\widetilde{x}, \widetilde{v}) \mathrm{d} \tilde{v} \mathrm{~d} \tilde{x} \mathrm{~d} t .
$$

Proof. As $\left(\widetilde{\phi}^{\varepsilon_{k}}\right)_{k}, \tilde{\phi}$ are uniformly locally bounded on $[0, T] \times \mathbb{R}^{2} \times \mathbb{R}^{2}$ cf. Remarks 3.4, 3.6, we may assume, without loss of generality, that $\psi \in C_{c}^{1}\left(\mathbb{R}_{+} \times \mathbb{R}^{2} \times \mathbb{R}^{2}\right)$. Pick a $C^{1}$ test function $\psi$ with support contained in $[0, T] \times B_{R}$ and observe that

$$
\begin{aligned}
\int_{\mathbb{R}_{+}} \int_{\mathbb{R}^{2}} \int_{\mathbb{R}^{2}} \phi^{\varepsilon_{k}} \psi \mathrm{d} \tilde{v} \mathrm{~d} \tilde{x} \mathrm{~d} t & =\int_{\mathbb{R}_{+}} \int_{\mathbb{R}^{2}} \int_{\mathbb{R}^{2}} \phi^{\varepsilon_{k}}\left(t, \widetilde{x}-\omega_{c}^{-1} \mathcal{R}\left(-\omega_{c} t / \varepsilon_{k}\right){ }^{\perp} \widetilde{v}\right) \psi \mathrm{d} \tilde{v} \mathrm{~d} \tilde{x} \mathrm{~d} t \\
& =\int_{\mathbb{R}_{+}} \int_{\mathbb{R}^{2}} \int_{\mathbb{R}^{2}} \tilde{f}^{\varepsilon_{k}}(t, \widetilde{y}, \widetilde{w}) \beta_{k}(t, \widetilde{y}, \widetilde{w}) \mathrm{d} \tilde{w} \mathrm{~d} \tilde{y} \mathrm{~d} t
\end{aligned}
$$


where for any $(t, \widetilde{y}, \widetilde{w}) \in \mathbb{R}_{+} \times \mathbb{R}^{2} \times \mathbb{R}^{2}$

$$
\begin{aligned}
\beta_{k}(t, \widetilde{y}, \widetilde{w}) & =\int_{\mathbb{R}^{2}} \int_{\mathbb{R}^{2}} \psi(t, \widetilde{x}, \widetilde{v}) e\left(\widetilde{x}-\widetilde{y}-\omega_{c}^{-1} \mathcal{R}\left(-\omega_{c} t / \varepsilon_{k}\right)^{\perp}(\widetilde{v}-\widetilde{w})\right) \mathrm{d} \tilde{v} \mathrm{~d} \tilde{x} \\
& =\int_{\mathbb{R}^{2}} e(\widetilde{z}) \int_{\mathbb{R}^{2}} \psi\left(t, \widetilde{y}+\widetilde{z}+\omega_{c}^{-1} \mathcal{R}\left(-\omega_{c} t / \varepsilon_{k}\right)^{\perp}(\widetilde{v}-\widetilde{w}), \widetilde{v}\right) \mathrm{d} \tilde{v} \mathrm{~d} \widetilde{z}
\end{aligned}
$$

Since $e=e(z)$ is locally integrable on $\mathbb{R}^{2}$, it is easily seen that $\left(\beta_{k}\right)_{k}$ remains into a bounded set of $L^{\infty}\left([0, T] ; H_{0}^{1}\left(B_{R}\right)\right)$, for any $T, R \in \mathbb{R}_{+}$, and thus thanks to the strong convergence of $\left(\tilde{f}^{\varepsilon_{k}}\right)_{k}$ in $L^{\infty}\left([0, T] ; H^{-1}\left(B_{R}\right)\right)$, combined to Proposition 3.2 , we obtain as in the proof of Theorem 1.1

$$
\begin{aligned}
\lim _{k \rightarrow+\infty} & \int_{\mathbb{R}_{+}} \int_{\mathbb{R}^{2}} \int_{\mathbb{R}^{2}} \phi^{\varepsilon k} \psi \mathrm{d} \tilde{v} \mathrm{~d} \tilde{x} \mathrm{~d} t=\int_{\mathbb{R}_{+}} \int_{\mathbb{R}^{2}} \int_{\mathbb{R}^{2}} \tilde{f}(t, \widetilde{y}, \widetilde{w}) \int_{\mathbb{R}^{2}} \int_{\mathbb{R}^{2}} \psi(t, \widetilde{x}, \widetilde{v}) \\
& \times \frac{1}{T_{c}} \int_{0}^{T_{c}} e\left(\widetilde{x}-\widetilde{y}-\omega_{c}^{-1} \mathcal{R}\left(-\omega_{c} s\right)^{\perp}(\widetilde{v}-\widetilde{w})\right) \mathrm{d} s \mathrm{~d} \tilde{v} \mathrm{~d} \tilde{x} \mathrm{~d} \tilde{w} \mathrm{~d} \tilde{y} \mathrm{~d} t \\
& =\int_{\mathbb{R}_{+}} \int_{\mathbb{R}^{2}} \int_{\mathbb{R}^{2}} \tilde{\phi}[\tilde{f}(t)](\widetilde{x}, \widetilde{v}) \psi(t, \widetilde{x}, \widetilde{v}) \mathrm{d} \tilde{v} \mathrm{~d} \tilde{x} \mathrm{~d} t .
\end{aligned}
$$

We have to check that the function

$$
I_{6}(\widetilde{z}, \widetilde{v}, \widetilde{y}, \widetilde{w}, t, s):=\tilde{f}(t, \widetilde{y}, \widetilde{w}) e(\widetilde{z}) \psi\left(t, \widetilde{y}+\widetilde{z}+\omega_{c}^{-1} \mathcal{R}\left(-\omega_{c} s\right)^{\perp}(\widetilde{v}-\widetilde{w}), \widetilde{v}\right)
$$

belongs to $L^{1}\left(\mathbb{R}^{8} \times \mathbb{R}_{+} ; C_{\#}\left(\mathbb{R}_{s}\right)\right)$. For doing that, observe that

$$
\begin{aligned}
\left|\psi\left(t, \widetilde{y}+\widetilde{z}+\omega_{c}^{-1} \mathcal{R}\left(-\omega_{c} s\right)^{\perp}(\widetilde{v}-\widetilde{w}), \widetilde{v}\right)\right| & \leq\|\psi\|_{L^{\infty}} \mathbf{1}_{\{0 \leq t \leq T\}} \mathbf{1}_{\{|\widetilde{v}| \leq R\}} \mathbf{1}_{\left\{\left|\widetilde{y}+\widetilde{z}+\omega_{c}^{-1} \mathcal{R}\left(-\omega_{c} s\right) \perp(\widetilde{v}-\widetilde{w})\right| \leq R\right\}} \\
& \leq\|\psi\|_{L^{\infty}} \mathbf{1}_{\{0 \leq t \leq T\}} \mathbf{1}_{\{|\widetilde{v}| \leq R\}} \mathbf{1}_{\left\{|\widetilde{z}| \leq R\left(1+\left|\omega_{c}\right|^{-1}\right)+|\widetilde{y}|+\left|\omega_{c}\right|^{-1}|\widetilde{w}|\right\}}
\end{aligned}
$$

and therefore we can write

$$
\begin{aligned}
\int_{\mathbb{R}^{8}} \int_{\mathbb{R}_{+}} & \sup _{s}\left|I_{6}(\widetilde{z}, \widetilde{v}, \widetilde{y}, \widetilde{w}, t, s)\right| \mathrm{d} \widetilde{z} \mathrm{~d} \widetilde{v} \mathrm{~d} \widetilde{y} \mathrm{~d} \widetilde{w} \mathrm{~d} t \leq \int_{0}^{T} \int_{\mathbb{R}^{2}} \int_{\mathbb{R}^{2}} \tilde{f}(t, \widetilde{y}, \widetilde{w})\|\psi\|_{L^{\infty}} \\
& \times \int_{|\widetilde{v}| \leq R} \int_{\mathbb{R}^{2}}|e(\widetilde{z})| \mathbf{1}_{\left\{|\tilde{z}| \leq R\left(1+\left|\omega_{c}\right|^{-1}\right)+|\widetilde{y}|+\left|\omega_{c}\right|^{-1}|\widetilde{w}|\right\}} \mathrm{d} \widetilde{z} \mathrm{~d} \widetilde{v} \mathrm{~d} \tilde{w} \mathrm{~d} \tilde{y} \mathrm{~d} t \\
& \leq C\|\psi\|_{L^{\infty}} \int_{0}^{T} \int_{\mathbb{R}^{2}} \int_{\mathbb{R}^{2}} \tilde{f}(t, \widetilde{y}, \widetilde{w})\left(1+|\widetilde{y}|^{3}+|\widetilde{w}|^{3}\right) \mathrm{d} \tilde{w} \mathrm{~d} \tilde{y} \mathrm{~d} t<+\infty
\end{aligned}
$$

The second and third convergences are consequences of the first one (establish them for test functions $\psi \in C_{c}^{2}\left(\mathbb{R}_{+} \times \mathbb{R}^{2} \times \mathbb{R}^{2}\right)$ and then proceed by density arguments, using the uniform bounds (46), (47), (35)).

For the last convergence, we combine one more time the strong convergence of $\left(\tilde{f}^{\varepsilon_{k}}\right)_{k}$ in 
$L^{\infty}\left([0, T] ; H^{-1}\left(B_{R}\right)\right)$, for any $T, R \in \mathbb{R}_{+}$and Proposition 3.2. For any $\eta \in C_{c}^{0}\left(\mathbb{R}_{+}\right)$we obtain

$$
\begin{aligned}
\int_{\mathbb{R}_{+}} \eta(t) & \int_{\mathbb{R}^{2}}\left|\nabla_{x} \phi^{\varepsilon_{k}}(t, x)\right|^{2} \mathrm{~d} x \mathrm{~d} t=\int_{\mathbb{R}_{+}} \eta(t) \int_{\mathbb{R}^{2}} \int_{\mathbb{R}^{2}} f^{\varepsilon_{k}}(t, x, v) \phi^{\varepsilon_{k}}(t, x) \mathrm{d} v \mathrm{~d} x \mathrm{~d} t \\
& =\int_{\mathbb{R}_{+}} \eta(t) \int_{\mathbb{R}^{2}} \int_{\mathbb{R}^{2}} f^{\varepsilon_{k}}(t, x, v) \int_{\mathbb{R}^{2}} \int_{\mathbb{R}^{2}} f^{\varepsilon_{k}}(t, y, w) e(x-y) \mathrm{d} w \mathrm{~d} y \mathrm{~d} v \mathrm{~d} x \mathrm{~d} t \\
& =\int_{\mathbb{R}_{+}} \int_{\mathbb{R}^{2}} \int_{\mathbb{R}^{2}} \int_{\mathbb{R}^{2}} \int_{\mathbb{R}^{2}} \eta(t) \tilde{f}^{\varepsilon_{k}}(t, \widetilde{x}, \widetilde{v}) \tilde{f}^{\varepsilon_{k}}(t, \widetilde{y}, \widetilde{w}) \\
& \times e\left(\widetilde{x}-\widetilde{y}-\omega_{c}^{-1} \mathcal{R}\left(-\omega_{c} t / \varepsilon_{k}\right)^{\perp}(\widetilde{v}-\widetilde{w})\right) \mathrm{d} \tilde{w} \mathrm{~d} \tilde{y} \mathrm{~d} \tilde{v} \mathrm{~d} \tilde{x} \mathrm{~d} t \\
& \underset{k \rightarrow+\infty}{\longrightarrow} \int_{\mathbb{R}_{+}} \int_{\mathbb{R}^{2}} \int_{\mathbb{R}^{2}} \int_{\mathbb{R}^{2}} \int_{\mathbb{R}^{2}} \eta(t) \tilde{f}(t, \widetilde{x}, \widetilde{v}) \tilde{f}(t, \widetilde{y}, \widetilde{w}) \\
& \times \frac{1}{T_{c}} \int_{0}^{T_{c}} e\left(\widetilde{x}-\widetilde{y}-\omega_{c}^{-1} \mathcal{R}\left(-\omega_{c} s\right)^{\perp}(\widetilde{v}-\widetilde{w})\right) \mathrm{d} \tilde{w} \mathrm{~d} \tilde{y} \mathrm{~d} \tilde{v} \mathrm{~d} \tilde{x} \mathrm{~d} t \\
& =\int_{\mathbb{R}_{+}} \int_{\mathbb{R}^{2}} \int_{\mathbb{R}^{2}} \eta(t) \tilde{f}(t, \widetilde{x}, \widetilde{v}) \tilde{\phi}[\tilde{f}(t)](\widetilde{x}, \widetilde{v}) \mathrm{d} \tilde{v} \mathrm{~d} \tilde{x} \mathrm{~d} t .
\end{aligned}
$$

In particular we deduce that $t \rightarrow \int_{\mathbb{R}^{2}} \int_{\mathbb{R}^{2}} \tilde{f}(t, \tilde{x}, \widetilde{v}) \tilde{\phi}[\tilde{f}(t)](\widetilde{x}, \widetilde{v}) \mathrm{d} \tilde{v} \mathrm{~d} \tilde{x}$ is non negative on $\mathbb{R}_{+}$.

Remark 3.7 Clearly, the hypothesis on the support compactness for the initial density $f^{\text {in }}$ can be relaxed. For example, the first convergence in Proposition 3.4 holds true provided that the initial density has finite moments of order three, that is

$$
\int_{\mathbb{R}^{2}} \int_{\mathbb{R}^{2}} f^{\text {in }}(x, v)\left(|x|^{3}+|v|^{3}\right) \mathrm{d} \tilde{v} \mathrm{~d} \tilde{x}<+\infty .
$$

\section{The three dimensional setting}

We concentrate now on the three dimensional finite Larmor radius regime (2), (3), (4). We perform a formal analysis, by indicating the expected results. The fast dynamics appears only in the orthogonal directions, and we will see that the homogenization procedure can be reduced essentially to that of the two dimensional case. Filtering out the fast cyclotronic motion leads to the new densities $\tilde{f}^{\varepsilon}(t, \widetilde{x}, \widetilde{v})=f^{\varepsilon}(t, x, v)$ where

$$
\widetilde{x}=\left(\bar{x}+\frac{\perp \bar{v}}{\omega_{c}}, x_{3}\right), \widetilde{v}=\left(\mathcal{R}\left(\omega_{c} t / \varepsilon\right) \bar{v}, v_{3}\right) .
$$

Written in the new phase space coordinates, the Vlasov problem becomes

$$
\partial_{t} \tilde{f}^{\varepsilon}-\omega_{c}^{-1}{ }^{\perp} \nabla_{\bar{x}} \phi^{\varepsilon}(t, x) \cdot \nabla_{\overline{\widetilde{x}}} \tilde{f}^{\varepsilon}+\widetilde{v}_{3} \partial_{\widetilde{x}_{3}} \tilde{f}^{\varepsilon}-\mathcal{R}\left(\omega_{c} t / \varepsilon\right) \nabla_{\bar{x}} \phi^{\varepsilon}(t, x) \cdot \nabla_{\overline{\widetilde{v}}} \tilde{f}^{\varepsilon}-\varepsilon \partial_{x_{3}} \phi^{\varepsilon} \partial_{\widetilde{v}_{3}} \tilde{f}^{\varepsilon}=0
$$

with $x=\left(\overline{\widetilde{x}}-\omega_{c}^{-1} \mathcal{R}\left(-\omega_{c} t / \varepsilon\right) \perp \overline{\widetilde{v}}, x_{3}\right)$, together with the initial condition

$$
\tilde{f}^{\varepsilon}(0, \widetilde{x}, \widetilde{v})=f^{\text {in }}\left(\overline{\widetilde{x}}-\frac{\perp \overline{\widetilde{v}}}{\omega_{c}}, \widetilde{x}_{3}, \widetilde{v}\right) .
$$


Solving the Poisson equation (3), one gets

$$
\phi^{\varepsilon}(t, x)=\frac{1}{4 \pi \varepsilon} \int_{\mathbb{R}^{3}} \frac{\rho^{\varepsilon}(t, y)}{\sqrt{|\bar{x}-\bar{y}|^{2}+\varepsilon^{-2}\left(x_{3}-y_{3}\right)^{2}}} \mathrm{~d} y=\frac{1}{4 \pi} \int_{\mathbb{R}^{3}} \frac{\rho^{\varepsilon}\left(t, \bar{y}, x_{3}+\varepsilon u\right)}{\sqrt{|\bar{x}-\bar{y}|^{2}+u^{2}}} \mathrm{~d} \bar{y} \mathrm{~d} u
$$

implying that

$$
\nabla_{\bar{x}} \phi^{\varepsilon}(t, x)=-\frac{1}{4 \pi} \int_{\mathbb{R}^{3}} \frac{\rho^{\varepsilon}\left(t, \bar{y}, x_{3}+\varepsilon u\right)}{\left(|\bar{x}-\bar{y}|^{2}+u^{2}\right)^{3 / 2}}(\bar{x}-\bar{y}) \mathrm{d} \bar{y} \mathrm{~d} u .
$$

Neglecting the variations of the charge density $\rho^{\varepsilon}$ along the $x_{3}$ axis, we obtain when $\varepsilon$ becomes small

$$
\begin{aligned}
\nabla_{\bar{x}} \phi^{\varepsilon}(t, x) & =-\frac{1}{4 \pi} \int_{\mathbb{R}^{2}} \rho^{\varepsilon}\left(t, \bar{y}, x_{3}\right)(\bar{x}-\bar{y}) \int_{\mathbb{R}} \frac{\mathrm{d} u}{\left(|\bar{x}-\bar{y}|^{2}+u^{2}\right)^{3 / 2}} \mathrm{~d} \bar{y}+o(1) \\
& =-\frac{1}{2 \pi} \int_{\mathbb{R}^{2}} \rho^{\varepsilon}\left(t, \bar{y}, x_{3}\right) \frac{\bar{x}-\bar{y}}{|\bar{x}-\bar{y}|^{2}} \mathrm{~d} \bar{y}+o(1) \\
& =\int_{\mathbb{R}^{2}} \nabla e(\bar{x}-\bar{y}) \rho^{\varepsilon}\left(t, \bar{y}, x_{3}\right) \mathrm{d} \bar{y}+o(1)
\end{aligned}
$$

since, by direct computation we check that $\int_{\mathbb{R}} \frac{\mathrm{d} u}{\left(a^{2}+u^{2}\right)^{3 / 2}}=\frac{2}{a^{2}}, a>0$. Notice that, at least formally, we have

$$
\begin{aligned}
\varepsilon \phi^{\varepsilon}(t, x) & =\frac{\varepsilon}{4 \pi} \int_{\mathbb{R}^{3}} \rho^{\varepsilon}(t, y) \frac{\partial}{\partial y_{3}} \ln \left[\frac{y_{3}-x_{3}}{\varepsilon}+\sqrt{|\bar{x}-\bar{y}|^{2}+\varepsilon^{-2}\left(x_{3}-y_{3}\right)^{2}}\right] \mathrm{d} y \\
& =-\frac{\varepsilon}{4 \pi} \int_{\mathbb{R}^{3}} \int_{\mathbb{R}^{3}} \partial_{y_{3}} f^{\varepsilon}(t, y, w) \ln \left[\frac{y_{3}-x_{3}}{\varepsilon}+\sqrt{|\bar{x}-\bar{y}|^{2}+\varepsilon^{-2}\left(x_{3}-y_{3}\right)^{2}}\right] \mathrm{d} w \mathrm{~d} y \\
& =-\frac{\varepsilon}{4 \pi} \int_{\mathbb{R}^{3}} \int_{\mathbb{R}^{3}} \partial_{\widetilde{y}_{3}} \tilde{f}^{\varepsilon}(t, \widetilde{y}, \widetilde{w}) \\
& \times \ln \left[\frac{\widetilde{y}_{3}-\widetilde{x}_{3}}{\varepsilon}+\sqrt{\left|\overline{\widetilde{x}}-\overline{\widetilde{y}}-\omega_{c}^{-1} \mathcal{R}\left(-\omega_{c} t / \varepsilon\right) \perp(\overline{\widetilde{v}}-\overline{\widetilde{w}})\right|^{2}+\varepsilon^{-2}\left(\widetilde{x}_{3}-\widetilde{y}_{3}\right)^{2}}\right] \mathrm{d} \widetilde{w} \mathrm{~d} \widetilde{y} \\
& \underset{\varepsilon \searrow 0}{\longrightarrow}
\end{aligned}
$$

as suggested by the pointwise convergence

$$
\lim _{\varepsilon \searrow 0} \varepsilon \ln \left[\frac{\widetilde{y}_{3}-\widetilde{x}_{3}}{\varepsilon}+\sqrt{\left|\overline{\widetilde{x}}-\overline{\widetilde{y}}-\omega_{c}^{-1} \mathcal{R}\left(-\omega_{c} t / \varepsilon\right)^{\perp}(\overline{\widetilde{v}}-\overline{\widetilde{w}})\right|^{2}+\varepsilon^{-2}\left(\widetilde{x}_{3}-\widetilde{y}_{3}\right)^{2}}\right]=0
$$

at any point such that $|\overline{\widetilde{x}}-\overline{\widetilde{y}}| \neq|\overline{\widetilde{v}}-\overline{\widetilde{w}}| /\left|\omega_{c}\right|$. Neglecting also the parallel electric field we obtain the problem

$$
\partial_{t} \tilde{f}^{\varepsilon}-\omega_{c}^{-1}{ }^{\perp} \nabla_{\bar{x}} \phi^{\varepsilon}(t, x) \cdot \nabla_{\bar{x}} \tilde{f}^{\varepsilon}+\widetilde{v}_{3} \partial_{\widetilde{x}_{3}} \tilde{f}^{\varepsilon}-\mathcal{R}\left(\omega_{c} t / \varepsilon\right) \nabla_{\bar{x}} \phi^{\varepsilon}(t, x) \cdot \nabla_{\widetilde{v}} \tilde{f}^{\varepsilon}=o(1)
$$

with

$$
\begin{aligned}
\nabla_{\bar{x}} \phi^{\varepsilon}(t, x) & =\int_{\mathbb{R}^{2}} \nabla e(\bar{x}-\bar{y}) \rho^{\varepsilon}\left(t, \bar{y}, x_{3}\right) \mathrm{d} \bar{y} \\
& =\nabla_{\overline{\widetilde{x}}} \int_{\mathbb{R}^{2}} \int_{\mathbb{R}^{3}} e\left(\overline{\widetilde{x}}-\overline{\widetilde{y}}-\omega_{c}^{-1} \mathcal{R}\left(-\omega_{c} t / \varepsilon\right)^{\perp}(\overline{\widetilde{v}}-\overline{\widetilde{w}})\right) \tilde{f}^{\varepsilon}\left(t, \overline{\widetilde{y}}, x_{3}, \widetilde{w}\right) \mathrm{d} \widetilde{w} \mathrm{~d} \overline{\widetilde{y}} .
\end{aligned}
$$

Based on the two dimensional analysis, we expect that the limit density $\tilde{f}=\lim _{\varepsilon} \searrow_{0} \tilde{f}^{\varepsilon}$ solves the problem announced in Theorem 1.2. 


\section{A Average fundamental solution of the Laplace operator}

Proof. (of Proposition 2.2)

1. If $\xi=0$ and $\eta \neq 0$ we are done since we have

$$
\mathcal{E}(0, \eta)=\frac{1}{2 \pi} \int_{0}^{2 \pi} e\left(-\frac{\mathcal{R}(\theta)}{\omega_{c}} \eta\right) \mathrm{d} \theta=\frac{1}{2 \pi} \int_{0}^{2 \pi} e\left(\frac{\eta}{\omega_{c}}\right) \mathrm{d} \theta=e\left(\frac{\eta}{\omega_{c}}\right)
$$

Assume now that $\xi \neq 0$ and let us observe that $\mathcal{E}(\xi, \eta)=F\left(\xi,|\eta| /\left|\omega_{c}\right|\right)$, where

$$
\mathcal{F}(\xi, r)= \begin{cases}\frac{1}{2 \pi r} \int_{|z-\xi|=r} e(z) \mathrm{d} \sigma(z), & \text { if } r>0 \\ e(\xi), & \text { if } r=0 .\end{cases}
$$

For any $r \in] 0,|\xi|[$ we have

$$
\begin{aligned}
\frac{\mathrm{d}}{\mathrm{d} r} \mathcal{F}(\xi, r) & =\frac{\mathrm{d}}{\mathrm{d} r} \frac{1}{2 \pi} \int_{|y|=1} e(\xi+r y) \mathrm{d} \sigma(y) \\
& =\frac{1}{2 \pi} \int_{|y|=1} \nabla e(\xi+r y) \cdot y \mathrm{~d} \sigma(y) \\
& =\frac{1}{2 \pi r} \int_{|z-\xi|=r} \nabla e(z) \cdot \frac{z-\xi}{r} \mathrm{~d} \sigma(z) \\
& =\frac{1}{2 \pi r} \int_{|z-\xi|<r} \Delta e(z) \mathrm{d} z=0 .
\end{aligned}
$$

Therefore the function $r \rightarrow \mathcal{F}(\xi, r)$ is constant on $] 0,|\xi|[$

$$
F(\xi, r)=\lim _{r^{\prime} \backslash 0} F\left(\xi, r^{\prime}\right)=e(\xi)=F(\xi, 0), \quad 0<r<|\xi| .
$$

The value of $\mathcal{F}(\xi,|\xi|)$ follows by direct computation

$$
\begin{aligned}
F(\xi,|\xi|) & =\frac{1}{2 \pi} \int_{-\pi}^{\pi} e(|\xi|(1-\cos \theta,-\sin \theta)) \mathrm{d} \theta \\
& =\frac{1}{\pi} \int_{0}^{\pi}\left(-\frac{1}{2 \pi}\right) \ln \left(|\xi| 2 \sin \frac{\theta}{2}\right) \mathrm{d} \theta \\
& =e(\xi)-\frac{1}{2 \pi^{2}} \int_{0}^{\pi} \ln \left(2 \sin \frac{\theta}{2}\right) \mathrm{d} \theta=e(\xi)
\end{aligned}
$$

where we have used the Euler integral $\int_{0}^{\pi / 2} \ln \sin x \mathrm{~d} x=-\frac{\pi}{2} \ln 2$. Therefore the function $r \rightarrow \mathcal{F}(\xi, r)$ is constant on $[0,|\xi|]$. If $r>|\xi|$, the function $e(\cdot)$ has a singularity inside the disk of center $\xi$ and radius $r$ and therefore, we have for any $\delta$ small enough

$$
\begin{aligned}
\frac{\mathrm{d}}{\mathrm{d} r} \mathcal{F}(\xi, r) & =\frac{1}{2 \pi r} \int_{|z-\xi|=r} \nabla e(z) \cdot \frac{z-\xi}{r} \mathrm{~d} \sigma(z) \\
& =\frac{1}{2 \pi r} \int_{|z|=\delta} \nabla e(z) \cdot \frac{z}{\delta} \mathrm{d} \sigma(z)=-\frac{1}{2 \pi r} .
\end{aligned}
$$


Therefore, for any $r \geq|\xi|$ we obtain

$$
\mathcal{F}(\xi, r)=F(\xi,|\xi|)-\int_{|\xi|}^{r} \frac{\mathrm{d} u}{2 \pi u}=-\frac{1}{2 \pi} \ln r .
$$

Combining (49), (50) yields

$$
\mathcal{F}(\xi, r)=-\frac{1}{2 \pi} \ln r \mathbf{1}_{\{|\xi| \leq r\}}+e(\xi) \mathbf{1}_{\{|\xi|>r\}}
$$

saying that

$$
\mathcal{E}(\xi, \eta)=\mathcal{F}\left(\xi, \frac{|\eta|}{\left|\omega_{c}\right|}\right)=e\left(\frac{\eta}{\omega_{c}}\right) \mathbf{1}_{\left\{|\xi| \leq|\eta| /\left|\omega_{c}\right|\right\}}+e(\xi) \mathbf{1}_{\left\{|\xi|>|\eta| /\left|\omega_{c}\right|\right\}}
$$

2. Notice that the functions $\mathcal{E}(\xi, \eta), \nabla e(\xi) \mathbf{1}_{\left\{|\xi|>|\eta| /\left|\omega_{c}\right|\right\}}, \omega_{c}^{-1} \nabla e\left(\frac{\eta}{\omega_{c}}\right) \mathbf{1}_{\left\{|\xi| \leq|\eta| /\left|\omega_{c}\right|\right\}}$ are locally integrable on $\mathbb{R}^{2} \times \mathbb{R}^{2}$, and therefore our statement makes sense. Pick a function $\varphi \in C_{c}^{1}\left(\mathbb{R}^{2} \times\right.$ $\mathbb{R}^{2}$ ) and observe that

$$
\begin{aligned}
-\int_{\mathbb{R}^{2} \times \mathbb{R}^{2}} \mathcal{E} & (\xi, \eta) \nabla_{\xi} \varphi \mathrm{d}(\xi, \eta)=-\int_{|\xi| \leq \frac{|\eta|}{\left|\omega_{c}\right|}} e\left(\frac{\eta}{\omega_{c}}\right) \nabla_{\xi} \varphi \mathrm{d}(\xi, \eta)-\int_{|\xi|>\frac{|\eta|}{\left|\omega_{c}\right|}} e(\xi) \nabla_{\xi} \varphi \mathrm{d}(\xi, \eta) \\
= & -\int_{\mathbb{R}^{2}} e\left(\frac{\eta}{\omega_{c}}\right) \int_{|\xi| \leq \frac{|\eta|}{\left|\omega_{c}\right|}} \nabla_{\xi} \varphi \mathrm{d} \xi \mathrm{d} \eta-\int_{\mathbb{R}^{2}} \int_{|\xi|>\frac{|\eta|}{\left|\omega_{c}\right|}} e(\xi) \nabla_{\xi} \varphi \mathrm{d} \xi \mathrm{d} \eta \\
& =-\int_{\mathbb{R}^{2}} e\left(\frac{\eta}{\omega_{c}}\right) \int_{|\xi|=\frac{|\eta|}{\left|\omega_{c}\right|}} \varphi \frac{\xi}{|\xi|} \mathrm{d} \sigma(\xi) \mathrm{d} \eta+\int_{\mathbb{R}^{2}} \int_{|\xi|=\frac{|\eta|}{\left|\omega_{c}\right|}} e(\xi) \varphi \frac{\xi}{|\xi|} \mathrm{d} \sigma(\xi) \mathrm{d} \eta \\
& +\int_{\mathbb{R}^{2}} \int_{|\xi|>\frac{|\eta|}{\left|\omega_{c}\right|}} \nabla e(\xi) \varphi(\xi, \eta) \mathrm{d} \xi \mathrm{d} \eta \\
& =\int_{\mathbb{R}^{2} \times \mathbb{R}^{2}} \nabla e(\xi) \mathbf{1}_{\left\{|\xi|>\frac{|\eta|}{\left|\omega_{c}\right|}\right\}} \varphi(\xi, \eta) \mathrm{d}(\xi, \eta) .
\end{aligned}
$$

Therefore we have $\nabla_{\xi} \mathcal{E}(\xi, \eta)=\nabla e(\xi) \mathbf{1}_{\left\{|\xi|>\frac{\mid \eta}{\mid \omega_{c}}\right\}}$ in $\mathcal{D}^{\prime}\left(\mathbb{R}^{2} \times \mathbb{R}^{2}\right)$. Similarly we obtain

$$
\begin{aligned}
-\int_{\mathbb{R}^{2} \times \mathbb{R}^{2}} \mathcal{E}(\xi, \eta) \nabla_{\eta} \varphi \mathrm{d}(\xi, \eta) & =-\int_{|\xi| \leq \frac{|\eta|}{\left|\omega_{c}\right|}} e\left(\frac{\eta}{\omega_{c}}\right) \nabla_{\eta} \varphi \mathrm{d}(\xi, \eta)-\int_{|\xi|>\frac{|\eta|}{\left|\omega_{c}\right|}} e(\xi) \nabla_{\eta} \varphi \mathrm{d}(\xi, \eta) \\
& =-\int_{\mathbb{R}^{2}} \int_{|\xi| \leq \frac{|\eta|}{\left|\omega_{c}\right|}} e\left(\frac{\eta}{\omega_{c}}\right) \nabla_{\eta} \varphi \mathrm{d} \eta \mathrm{d} \xi-\int_{\mathbb{R}^{2}} e(\xi) \int_{|\xi|>\frac{|\eta|}{\left|\omega_{c}\right|}} \nabla_{\eta} \varphi \mathrm{d} \eta \mathrm{d} \xi \\
& =\int_{\mathbb{R}^{2} \times \mathbb{R}^{2}} \frac{1}{\omega_{c}} \nabla e\left(\frac{\eta}{\omega_{c}}\right) \mathbf{1}_{\left\{|\xi| \leq \frac{|\eta|}{\left|\omega_{c}\right|}\right\}} \varphi(\xi, \eta) \mathrm{d}(\xi, \eta)
\end{aligned}
$$

saying that $\nabla_{\eta} \mathcal{E}(\xi, \eta)=\omega_{c}^{-1} \nabla e\left(\frac{\eta}{\omega_{c}}\right) \mathbf{1}_{\left\{|\xi| \leq \frac{\mid \eta}{\mid \omega_{c}}\right\}}$ in $\mathcal{D}^{\prime}\left(\mathbb{R}^{2} \times \mathbb{R}^{2}\right)$.

3. Pick a function $\varphi \in C_{c}^{2}\left(\mathbb{R}^{2} \times \mathbb{R}^{2}\right)$ and observe that for any $i, j \in\{1,2\}$ we have

$$
\begin{aligned}
\left\langle\partial_{\xi_{i} \xi_{j}}^{2} \mathcal{E}, \varphi\right\rangle_{\mathcal{D}^{\prime}, \mathcal{D}} & =-\left\langle\partial_{\xi_{j}} \mathcal{E}, \partial_{\xi_{i}} \varphi\right\rangle_{\mathcal{D}^{\prime}, \mathcal{D}} \\
& =-\int_{\mathbb{R}^{2}} \int_{|\xi|>\frac{|\eta|}{\left|\omega_{c}\right|}} \partial_{j} e(\xi) \partial_{\xi_{i}} \varphi \mathrm{d} \xi \mathrm{d} \eta \\
& =\int_{\mathbb{R}^{2}} \int_{|\xi|>\frac{|\eta|}{\left|\omega_{c}\right|}} \partial_{i j}^{2} e(\xi) \varphi \mathrm{d} \xi \mathrm{d} \eta+\int_{\mathbb{R}^{2}} \int_{|\xi|=\frac{|\eta|}{\left|\omega_{c}\right|}} \partial_{j} e(\xi) \frac{\xi_{i}}{|\xi|} \varphi(\xi, \eta) \mathrm{d} \sigma(\xi) \mathrm{d} \eta
\end{aligned}
$$


Therefore we obtain

$$
\partial_{\xi}^{2} \mathcal{E}=-\left(I_{2}-2 \frac{\xi \otimes \xi}{|\xi|^{2}}\right) \frac{\mathbf{1}_{\left\{|\xi|>|\eta| /\left|\omega_{c}\right|\right\}} \mathrm{d}(\xi, \eta)}{2 \pi|\xi|^{2}}-\frac{\xi \otimes \xi}{|\xi|^{2}} \frac{\mathbf{1}_{\left\{|\xi|=|\eta| /\left|\omega_{c}\right|\right\}} \mathrm{d} \sigma(\xi, \eta)}{2 \pi|\xi| \sqrt{1+\omega_{c}^{-2}}}
$$

and in particular

$$
\Delta_{\xi} \mathcal{E}=-\frac{\mathbf{1}_{\left\{|\xi|=|\eta| /\left|\omega_{c}\right|\right\}} \mathrm{d} \sigma(\xi, \eta)}{2 \pi|\xi| \sqrt{1+\omega_{c}^{-2}}}
$$

Similarly we have

$$
\begin{aligned}
\left\langle\partial_{\xi_{i} \eta_{j}}^{2} \mathcal{E}, \varphi\right\rangle_{\mathcal{D}^{\prime}, \mathcal{D}} & =-\left\langle\partial_{\eta_{j}} \mathcal{E}, \partial_{\xi_{i}} \varphi\right\rangle_{\mathcal{D}^{\prime}, \mathcal{D}} \\
& =-\frac{1}{\omega_{c}} \int_{\mathbb{R}^{2}} \partial_{j} e\left(\frac{\eta}{\omega_{c}}\right) \int_{|\xi| \leq \frac{|\eta|}{\left|\omega_{c}\right|}} \partial_{\xi_{i}} \varphi \mathrm{d} \xi \mathrm{d} \eta \\
& =-\frac{1}{\omega_{c}} \int_{\mathbb{R}^{2}} \partial_{j} e\left(\frac{\eta}{\omega_{c}}\right) \int_{|\xi|=\frac{|\eta|}{\left|\omega_{c}\right|}} \varphi(\xi, \eta) \frac{\xi_{i}}{|\xi|} \mathrm{d} \sigma(\xi) \mathrm{d} \eta \\
& =\frac{1}{\omega_{c}} \int_{|\xi|=\frac{|\eta|}{\left|\omega_{c}\right|}} \frac{\eta_{j} \xi_{i} \varphi(\xi, \eta)}{2 \pi|\eta|^{2}|\xi| \sqrt{1+\omega_{c}^{-2}}} \mathrm{~d} \sigma(\xi, \eta)
\end{aligned}
$$

implying that

$$
\left(\nabla_{\xi} \otimes \nabla_{\eta}\right) \mathcal{E}={ }^{t}\left(\nabla_{\eta} \otimes \nabla_{\xi}\right) \mathcal{E}=\frac{\xi \otimes \eta}{|\xi||\eta|} \frac{1_{\left\{|\xi|=|\eta| /\left|\omega_{c}\right|\right\}}}{2 \pi \omega_{c}|\eta| \sqrt{1+\omega_{c}^{-2}}} \mathrm{~d} \sigma(\xi, \eta)
$$

Finally we write

$$
\begin{aligned}
\left\langle\partial_{\eta_{i} \eta_{j}}^{2} \mathcal{E}, \varphi\right\rangle_{\mathcal{D}^{\prime}, \mathcal{D}} & =-\left\langle\partial_{\eta_{j}} \mathcal{E}, \partial_{\eta_{i}} \varphi\right\rangle_{\mathcal{D}^{\prime}, \mathcal{D}} \\
& =-\frac{1}{\omega_{c}} \int_{\mathbb{R}^{2}} \int_{|\xi| \leq \frac{|\eta|}{\left|\omega_{c}\right|}} \partial_{j} e\left(\frac{\eta}{\omega_{c}}\right) \partial_{\eta_{i}} \varphi \mathrm{d} \eta \mathrm{d} \xi \\
& =\int_{\mathbb{R}^{2}} \int_{|\xi| \leq \frac{|\eta|}{\left|\omega_{c}\right|}} \partial_{i j}^{2} e(\eta) \varphi(\xi, \eta) \mathrm{d} \eta \mathrm{d} \xi+\int_{\mathbb{R}^{2}} \int_{|\xi|=\frac{|\eta|}{\left|\omega_{c}\right|}} \partial_{j} e(\eta) \frac{\eta_{i}}{|\eta|} \varphi \mathrm{d} \sigma(\eta) \mathrm{d} \xi
\end{aligned}
$$

and thus

$$
\partial_{\eta}^{2} \mathcal{E}=-\left(I_{2}-2 \frac{\eta \otimes \eta}{|\eta|^{2}}\right) \frac{\mathbf{1}_{\left\{|\xi| \leq|\eta| /\left|\omega_{c}\right|\right\}} \mathrm{d}(\xi, \eta)}{2 \pi|\eta|^{2}}-\frac{\eta \otimes \eta}{|\eta|^{2}} \frac{\mathbf{1}_{\left\{|\xi|=|\eta| /\left|\omega_{c}\right|\right\}} \mathrm{d} \sigma(\xi, \eta)}{2 \pi|\eta| \sqrt{1+\omega_{c}^{2}}}
$$

In particular we obtain

$$
\Delta_{\eta} \mathcal{E}=-\frac{\mathbf{1}_{\left\{|\xi|=|\eta| /\left|\omega_{c}\right|\right\}} \mathrm{d} \sigma(\xi, \eta)}{2 \pi|\eta| \sqrt{1+\omega_{c}^{2}}}
$$

\section{Acknowledgement}

The authors are thankful to M. Hauray for fruitful discussions and helpful remarks.

This work has been carried out within the framework of the EUROfusion Consortium and has received funding from the Euratom research and training programme 2014-2018 under 
grant agreement No 633053. The views and opinions expressed herein do not necessarily reflect those of the European Commission.

\section{References}

[1] G. Allaire, Homogenization and two-scale convergence, SIAM J. Math. Anal. 23(1992) 1482-1518.

[2] A.A. Arsen'ev, Global existence of weak solution of Vlasov's system of equations, Z. Vychisl. Mat. Fiz. 15(1975) 136-147.

[3] T. Aubin, Un théorème de compacité, C. R. Acad. Sci. Paris Sér. I Math. 256(1963) 5042-5044.

[4] M. Bostan, Weak solutions for the Vlasov-Poisson initial-boundary value problem with bounded electric field, Chin. Ann. Math. Ser. B (28)2007 389-420.

[5] M. Bostan, The Vlasov-Poisson system with strong external magnetic field. Finite Larmor radius regime, Asymptot. Anal., 61(2009) 91-123.

[6] M. Bostan, Transport equations with disparate advection fields. Application to the gyrokinetic models in plasma physics, J. Differential Equations 249(2010) 1620-1663.

[7] M. Bostan, Gyro-kinetic Vlasov equation in three dimensional setting. Second order approximation, SIAM J. Multiscale Model. Simul. 8(2010) 1923-1957.

[8] M. Bostan, A. Finot, M. Hauray, The effective Vlasov-Poisson system for strongly magnetized plasmas, C. R. Acad. Sci. Paris, Ser. I(2016), http://dx.doi.org/10.1016/j.crma.2016.04.014

[9] R. J. Diperna and P.-L. Lions, Ordinary differential equations, transport theory and Sobolev spaces, Invent. Math. 98(1989) 511-547.

[10] E. Frénod, E. Sonnendrücker, Homogenization of the Vlasov equation and of the VlasovPoisson system with strong external magnetic field, Asymptotic Anal. 18(1998) 193-213.

[11] E. Frénod, E. Sonnendrücker, The finite Larmor radius approximation, SIAM J. Math. Anal. 32(2001) 1227-1247. 
[12] E. Horst, Global strong solutions of Vlasov's equation. Necessary and sufficient conditions for their existence, Partial Differential Equations, Banach Cent. Publ. 19(1987) 143-153.

[13] D. Han-Kwan, Effect of the polarization drift in a strongly magnetized plasma, ESAIM : Math. Model. Numer. Anal. 46(2012) 1929-947.

[14] P.-L. Lions, B. Perthame, Propagation of moments and regularity for the 3-dimensional Vlasov-Poisson system, Invent. Math. 105(1991) 415-430.

[15] G. N'Guetseng, A general convergence result for a functional related to the theory of homogenization, SIAM J. Math. Anal. 20(1989) 608-623.

[16] J. Simon, Compact sets in the space $L^{p}(0, T ; B)$, Ann. Mat. Pura Appl. 146(1987) 65-96. 